MATHEMATICS OF COMPUTATION

Volume 68, Number 226, April 1999, Pages 487-517

S 0025-5718(99)01015-7

\title{
FINITE ELEMENT APPROXIMATION OF THE CAHN-HILLIARD EQUATION WITH CONCENTRATION DEPENDENT MOBILITY
}

\author{
JOHN W. BARRETT AND JAMES F. BLOWEY
}

\begin{abstract}
We consider the Cahn-Hilliard equation with a logarithmic free energy and non-degenerate concentration dependent mobility. In particular we prove that there exists a unique solution for sufficiently smooth initial data. Further, we prove an error bound for a fully practical piecewise linear finite element approximation in one and two space dimensions. Finally some numerical experiments are presented.
\end{abstract}

\section{INTRODUCTION}

Let $\Omega$ be a bounded domain in $\mathbf{R}^{d}, d \leq 3$, with a Lipschitz boundary $\partial \Omega$. We consider the Cahn-Hilliard equation with non-constant mobility and logarithmic free energy:

Find $\{u(x, t), w(x, t)\}$ such that

$$
\begin{aligned}
\frac{\partial u}{\partial t} & =\nabla \cdot(b(u) \nabla w) & & \text { in } \Omega_{T}:=\Omega \times(0, T), \\
w & =\Psi^{\prime}(u)-\gamma \Delta u & & \text { in } \Omega_{T}, \\
u(x, 0) & =u_{0}(x) & & \forall x \in \Omega, \\
\frac{\partial u}{\partial \nu} & =\frac{\partial w}{\partial \nu}=0 & & \text { on } \partial \Omega \times(0, T),
\end{aligned}
$$

where $\nu$ is normal to $\partial \Omega$. The mobility $b \in C[-1,1]$ is such that

$$
0 \leq b_{\min } \leq b(s) \leq b_{\max } \quad \forall s \in[-1,1] .
$$

The free energy $\Psi:[-1,1] \rightarrow \mathbf{R}$ is given by

$$
\Psi(s):=\psi(s)+\frac{\theta_{c}}{2}\left(1-s^{2}\right):=\frac{\theta}{2}\left[(1+s) \ln \left[\frac{1+s}{2}\right]+(1-s) \ln \left[\frac{1-s}{2}\right]\right]+\frac{\theta_{c}}{2}\left(1-s^{2}\right)
$$

and $\gamma, \theta$ and $\theta_{c}$ are positive constants with $\theta<\theta_{c}$. We define the monotone function $\phi:(-1,1) \rightarrow \mathbf{R}$ to be

$$
\phi(s):=\psi^{\prime}(s) \equiv \frac{\theta}{2}[\ln (1+s)-\ln (1-s)] .
$$

The above problem models phase separation of a binary mixture, which is quenched into an unstable state. Here $u:=X_{B}-X_{A} \in[-1,1]$, where $X_{A}, X_{B} \in$ $[0,1]$ are the mass fractions of the two components $A$ and $B$. When the quench is shallow, that is $\theta$ is close to $\theta_{c}$, then the free energy, $\Psi$, is usually approximated by a quartic polynomial. The majority of the mathematics literature has concentrated on this case, with constant mobility. However, this approximation is invalid if the

Received by the editor July 16, 1996 and, in revised form, September 16, 1997.

1991 Mathematics Subject Classification. Primary 65M60, 65M15, 35K55, 35K35, 82C26. 
quench is deep, i.e., $\theta \ll \theta_{c}$. For a fuller discussion of the model, see [10] and the references therein.

A mobility dependent on the concentration, $u$, appeared in the original derivation of the Cahn-Hilliard equation, see [8], and a thermodynamically reasonable choice is $b(s):=\left[1-s^{2}\right]_{+}$, see [13] and the references therein. This specific choice for $b$ leads to a number of mathematical difficulties since it is degenerate, i.e., $b_{\min }=0$ in (1.1). A simpler model is to consider for example

$$
b(s):= \begin{cases}1-\sigma s^{2} & \text { if }|s| \leq 1 \\ \frac{1}{2}(1-\sigma)\left[1+e^{\left.-\frac{2 \sigma\left(s^{2}-1\right)}{1-\sigma}\right]}\right] & \text { if }|s| \geq 1\end{cases}
$$

for a given $\sigma \in[0,1]$. Here, $\sigma=0$ yields a constant mobility, $\sigma \in(0,1)$ yields a non-degenerate concentration dependent mobility, and $\sigma=1$ yields the degenerate mobility mentioned above. For the purposes of the analysis in this paper, we have extended $b$ to $\mathbf{R}$, so that $b \in C^{1}(\mathbf{R})$ and is non-degenerate over $\mathbf{R}$ if $\sigma<1$. Throughout the paper, we will assume that

$$
b \in C(\mathbf{R}), \quad 0 \leq b_{\min } \leq b(s) \leq b_{\max } \quad \forall s \in \mathbf{R} .
$$

For the majority of our results we require the further restrictions

$$
b_{\text {min }}>0, \quad b \in C^{1}(\mathbf{R}) \quad \text { with } \quad\left|b^{\prime}(s)\right| \leq C \quad \forall s \in \mathbf{R} .
$$

We introduce a weak formulation of the above problem:

(P). Find $\{u, w\}$ such that $u(\cdot, 0)=u_{0}(\cdot)$ and for a.e. $t \in(0, T)$

$$
\begin{gathered}
\left\langle\frac{\partial u}{\partial t}, \eta\right\rangle+(b(u) \nabla w, \nabla \eta)=0 \quad \forall \eta \in H^{1}(\Omega), \\
(w, \eta)=\gamma(\nabla u, \nabla \eta)+\left(\Psi^{\prime}(u), \eta\right) \quad \forall \eta \in H^{1}(\Omega) .
\end{gathered}
$$

We have adopted the standard notation for Sobolev spaces, denoting the norm of $W^{m, p}(\Omega)(m \in \mathbf{N}, p \in[1, \infty])$ by $\|\cdot\|_{m, p}$ and semi-norm by $|\cdot|_{m, p}$. For $p=$ $2, W^{m, 2}(\Omega)$ will be denoted by $H^{m}(\Omega)$ with the associated norm and semi-norm written as $\|\cdot\|_{m}$ and $|\cdot|_{m}$, respectively. Throughout, $(\cdot, \cdot)$ denotes the standard $L^{2}$ inner product over $\Omega$ and $\langle\cdot, \cdot\rangle$ denotes the duality pairing between $\left(H^{1}(\Omega)\right)^{\prime}$ and $H^{1}(\Omega)$. In addition we define

$$
f \eta:=\frac{1}{|\Omega|}(\eta, 1) \quad \forall \eta \in L^{2}(\Omega) .
$$

There are two major difficulties in studying problem $(\mathrm{P})$. One is that $\psi^{\prime}(s)$ is singular at $s= \pm 1$ and therefore (1.5b) has no meaning if $u= \pm 1$ in an open set of non-zero measure. Secondly, establishing uniqueness of a solution is considerably more difficult when the mobility is concentration dependent.

Although, the Cahn-Hilliard equation has been extensively studied, very little mathematical work has appeared for a concentration dependent mobility. In [17] existence of generalized solutions in one spatial dimension is proved under the assumptions $\Psi \in C^{1}(\mathbf{R}), b \in C^{0, \beta}(\mathbf{R}), \beta \in(0,1)$, satisfying (1.1) and $u_{0} \in$ $H^{3}(\Omega)$. Furthermore, it is shown that if $b(-1)=b(1)=0$ and $\left\|u_{0}\right\|_{0, \infty} \leq 1$, then $\|u(\cdot, t)\|_{0, \infty} \leq 1, t \geq 0$. In [13] existence of solutions $\{u, w\}$ to (1.5a),(1.5b), satisfying (2.62) and (2.63), is proved under the assumptions $\Psi \in C^{1}(\mathbf{R})$ with $\Psi(s) \geq-C$, $\left|\Psi^{\prime}(s)\right| \leq C|s|^{r}+C\left(r \in \mathbf{R}^{+}\right.$for $d=1,2, r=3$ for $\left.d=3\right), b \in C(\mathbf{R})$ satisfying (1.1) with $b_{\text {min }}>0$ and $u_{0} \in H^{1}(\Omega)$. Furthermore, they prove existence when the mobility degenerates at \pm 1 and $\Psi^{\prime}$ has singularities at \pm 1 . This includes the case when $\Psi$ is defined as in (1.2) and $b(s):=\left[1-s^{2}\right]_{+}$. In [11] existence of a global unique strong solution $\{u, w\}$ to $(\mathrm{P}), u \in L^{2}\left(0, \infty ; H^{6}(\Omega)\right)$, is proved under the 
assumptions $\Psi$ is smooth, $b$ is smooth and nonnegative, $u_{0} \in H^{6}(\Omega)$ with $\left\|\Delta u_{0}\right\|_{2}$ sufficiently small and $b(m)>0, \Psi^{\prime \prime}(m) \geq 0$, i.e., metastable, where $m:=f u_{0}$. It is also shown that under these assumptions $u(\cdot, t) \rightarrow m$ in $L^{\infty}(\Omega)$ as $t \rightarrow \infty$.

We consider the finite element approximation of $(\mathrm{P})$ under the following assumptions on the mesh:

(A). Let $\Omega$ be a convex polyhedron. Let $\mathcal{T}^{h}$ be a quasi-uniform partitioning of $\Omega$ into disjoint open simplices $\kappa$ with $h_{\kappa}:=\operatorname{diam}(\kappa)$ and $h:=\max _{\kappa \in \mathcal{T} h} h_{\kappa}$, so that $\bar{\Omega}=\cup_{\kappa \in \mathcal{T} h} \bar{\kappa}$. In addition, it is assumed that $\mathcal{T}^{h}$ is an acute partitioning; that is for (i) $d=2$ the angle of any triangle does not exceed $\pi / 2$, (ii) $d=3$ the angle between any two faces of the same tetrahedron does not exceed $\pi / 2$. In fact the case $d=2$ can be relaxed to weakly acute, see [19]; that is, the sum of opposite angles relative to any side does not exceed $\pi$.

Associated with $\mathcal{T}^{h}$ is the finite element space

$$
S^{h}:=\left\{\chi \in C(\bar{\Omega}):\left.\chi\right|_{\kappa} \text { is linear } \forall \kappa \in \mathcal{T}^{h}\right\} \subset H^{1}(\Omega) .
$$

Let $\pi^{h}: C(\bar{\Omega}) \rightarrow S^{h}$ be the interpolation operator such that $\pi^{h} \eta\left(x_{j}\right)=\eta\left(x_{j}\right)$ $(j=1 \rightarrow J)$, where $\left\{x_{j}\right\}_{j=1}^{J}$ is the set of nodes of $\mathcal{T}^{h}$. A discrete inner product on $C(\bar{\Omega})$, is then defined by

$$
\left(\eta_{1}, \eta_{2}\right)^{h}:=\int_{\Omega} \pi^{h}\left(\eta_{1}(x) \eta_{2}(x)\right) \mathrm{d} x \equiv \sum_{j=1}^{J} M_{j} \eta_{1}\left(x_{j}\right) \eta_{2}\left(x_{j}\right),
$$

where $0<M_{j} \leq C h^{d}$. We introduce the $L^{2}$ projections $Q^{h}: L^{2}(\Omega) \rightarrow S^{h}$ and $\hat{Q}^{h}: L^{2}(\Omega) \rightarrow S^{h}$ defined by

$$
\left(Q^{h} \eta, \chi\right)=\left(\hat{Q}^{h} \eta, \chi\right)^{h}=(\eta, \chi) \quad \forall \chi \in S^{h} .
$$

Given $N$, a positive integer, let $\Delta t:=T / N$ denote the time step and $t_{n}:=n \Delta t$, $n=1 \rightarrow N$. We consider the following fully practical finite element approximation of $(\mathrm{P})$ :

$\left(\mathbf{P}^{h, \Delta t}\right)$. For $n=1 \rightarrow N$ find $\left\{U^{n}, W^{n}\right\} \in S^{h} \times S^{h}$ such that

$$
\begin{gathered}
\left(\frac{U^{n}-U^{n-1}}{\Delta t}, \chi\right)^{h}+b_{\max }\left(\nabla W^{n}, \nabla \chi\right) \\
=\left(\left[b_{\max }-b\left(U^{n-1}\right)\right] \nabla W^{n-1}, \nabla \chi\right) \quad \forall \chi \in S^{h}, \\
\left(W^{n}, \chi\right)^{h}=\gamma\left(\nabla U^{n}, \nabla \chi\right)+\left(\phi\left(U^{n}\right)-\theta_{c} U^{n}, \chi\right)^{h} \quad \forall \chi \in S^{h},
\end{gathered}
$$

where $U^{0} \equiv Q^{h} u_{0}$ or $\hat{Q}^{h} u_{0}$ and $W^{0} \in S^{h}$ is such that

$$
\left(W^{0}, \chi\right)^{h}=-\gamma\left(\Delta u_{0}, \chi\right)+\left(\phi\left(U^{0}\right)-\theta_{c} U^{0}, \chi\right)^{h} \quad \forall \chi \in S^{h} .
$$

The corresponding finite element approximation for constant mobility, i.e., $b_{\max }=$ $b_{\text {min }} \equiv b(s) \equiv 1$, is analysed in [2]. We note that the resulting nonlinear algebraic system that needs to be solved at each time level for $\left(\mathrm{P}^{h, \Delta t}\right)$ is the same that arises in the constant mobility case. It is the main purpose of this paper to extend the analysis in [2] to the non-constant mobility case and to prove the following error bound for the approximation $\left(\mathrm{P}^{h, \Delta t}\right)$.

Theorem 1.1. Let $u_{0} \in H^{3}(\Omega), \frac{\partial u_{0}}{\partial \nu}=0$ on $\partial \Omega$ and $\delta \in(0,1)$ be such that $\left\|u_{0}\right\|_{0, \infty} \leq 1-\delta$. Let the assumptions (A) hold and $\Delta t \equiv C h$, for any fixed 
constant $C$. Let $d \leq 2$ and $b$ satisfy (1.4a),(1.4b). Then for all $h>0$ such that $\Delta t \leq 4 \gamma /\left[b_{\max } \theta_{c}^{2}\right]$

$$
\left\|U^{0}\right\|_{0, \infty} \leq 1-\frac{1}{2} \delta \quad \text { and } \quad\left|u_{0}-U^{0}\right|_{1}^{2} \leq C h
$$

we have that

$$
\left\|u-U^{+}\right\|_{L^{2}\left(0, T ; H^{1}(\Omega)\right)}^{2}+\|u-U\|_{L^{\infty}\left(0, T ;\left(H^{1}(\Omega)\right)^{\prime}\right)}^{2} \leq C h,
$$

where for $n \geq 1$

$$
\begin{gathered}
U(\cdot, t):=\frac{t-t_{n-1}}{\Delta t} U^{n}(\cdot)+\frac{t_{n}-t}{\Delta t} U^{n-1}(\cdot) \quad t \in\left[t_{n-1}, t_{n}\right], \\
U^{+}(\cdot, t):=U^{n}(\cdot) \quad t \in\left(t_{n-1}, t_{n}\right] .
\end{gathered}
$$

We note that on choosing $U^{0} \equiv Q^{h} u_{0}$, the second bound in (1.9) is satisfied for all $h>0$ and a sufficient condition for the first to hold is that $h$ is sufficiently small. Whereas on choosing $U^{0} \equiv \hat{Q}^{h} u_{0}$ the first bound in (1.9) is satisfied for all $h>0$ and the second bound holds with further restrictions on $\mathcal{T}^{h}$ and $u_{0}$; e.g., on a uniform translation invariant mesh if $u_{0} \in W^{2, \infty}(\Omega)$.

The error bound in Theorem 1.1 is exactly that obtained for constant mobility in [2] under fewer restrictions on the data. However, it may not be optimal as the singular nonlinearity $\phi$ makes the analysis particularly delicate. Although our final error bound is only valid for the restricted data $d \leq 2$ with the mobility $b(\cdot)$ satisfying both (1.4a),(1.4b), a number of the results are developed for more general data. In particular, the approximation $\left(\mathrm{P}^{h, \Delta t}\right)$ is well posed for all $d \leq 3$ with $b(\cdot)$ satisfying just (1.4a). Similarly, the restrictions on the mesh in (A) of quasi-uniformity (required for the inverse estimates, see (3.11) and (3.10)) and weak acuteness (required only for the technical bound (3.17)) can be relaxed to a regular partitioning to prove well posedness of $\left(\mathrm{P}^{h, \Delta t}\right)$.

We note that one could consider an alternative time stepping scheme with $\theta_{c} U^{n}$ in (1.8b) replaced by $\theta_{c} U^{n-1}$. It is a simple matter to adapt the analysis is this paper to show that this scheme is unconditionally stable, and that the error estimate in Theorem 1.1 above holds for all $\Delta t \equiv C h$. However, the resulting scheme on eliminating $\left\{W^{n}\right\}_{n=0}^{N}$ is a three level time scheme for $\left\{U^{n}\right\}_{n=0}^{N}$. This leads to spurious modes and we have found that the stated discretization $\left(\mathrm{P}^{h, \Delta t}\right)$ performs better in practice.

The layout of this paper is as follows. In Section 2 we study a regularized problem $\left(\mathrm{P}_{\varepsilon}\right)$, where $\phi$ is replaced by $\phi_{\varepsilon}$. Firstly we prove some $\varepsilon$ independent stability bounds for the solution $\left\{u_{\varepsilon}, w_{\varepsilon}\right\}$, extending on those given in [16] and [2] for the constant mobility case. Passing to the limit, $\varepsilon=0$, we prove existence of a solution $\{u, w\}$ to $(\mathrm{P})$. Finally we prove uniqueness of these solutions to $\left(\mathrm{P}_{\varepsilon}\right)$ and $(\mathrm{P})$, and an error bound for this regularization procedure under a number of regularity assumptions, which are shown to hold for the restricted data $d \leq 2$ and $b(\cdot)$ satisfying both (1.4a),(1.4b). In Section 3 we prove firstly well posedness of a fully discrete continuous piecewise linear finite element approximation of $\left(\mathrm{P}_{\varepsilon}\right),\left(\mathrm{P}_{\varepsilon}^{h, \Delta t}\right)$, a regularized version of $\left(\mathrm{P}^{h, \Delta t}\right)$. Passing to the limit, $\varepsilon=0$, we prove the well posedness of $\left(\mathrm{P}^{h, \Delta t}\right)$. In addition we prove an error bound for the approximation $\left(\mathrm{P}_{\varepsilon}^{h, \Delta t}\right)$ of $\left(\mathrm{P}_{\varepsilon}\right)$ and this discrete regularization procedure for the restricted data $d \leq 2$ and $b(\cdot)$ satisfying both $(1.4 \mathrm{a}),(1.4 \mathrm{~b})$. By combining all the above error bounds and choosing the regularization parameter $\varepsilon$ and the time step $\Delta t$ in terms of the mesh spacing $h$, we obtain the error bound in Theorem 1.1. Throughout, $C$ denotes a generic constant independent of these three parameters. In addition $C\left(a_{1}, \cdots, a_{I}\right)$ 
denotes a constant depending on the non-negative parameters $\left\{a_{i}\right\}_{i=1}^{I}$, such that $C\left(a_{1}, \cdots, a_{I}\right) \leq C$ if $a_{i} \leq C$ for $i=1 \rightarrow I$. For notational convenience we write $C_{b} \equiv C\left(b_{\min }^{-1}\right)$. Finally in Section 4 we present some numerical experiments.

We end this section by noting that the error bound in [2] for the finite element approximation, $\left(\mathrm{P}^{h, \Delta t}\right)$, of $(\mathrm{P})$ with constant mobility has been extended to the multi-component version of $(\mathrm{P})$ with a constant mobility matrix in [3]. Furthermore, the existence proof in [13] for $(\mathrm{P})$ with a degenerate concentration dependent mobility has been extended to the multi-component version of $(\mathrm{P})$ with a degenerate concentration dependent mobility matrix in [14]. In a forthcoming paper we intend to extend the error bound in this paper to the multi-component version of (P) with a non-degenerate concentration dependent mobility matrix.

\section{A REgULARIZED PROBLEM}

In [16] Elliot and Luckhaus analysed (P) for constant mobility by introducing a regularized problem. We employ the same regularization procedure to study the case when the mobility is concentration dependent.

The logarithmic free energy $\Psi(\cdot)$ is replaced by the twice continuously differentiable function $\Psi_{\varepsilon}(s):=\psi_{\varepsilon}(s)+\frac{\theta_{c}}{2}\left(1-s^{2}\right)$, where $\varepsilon \in(0,1)$ and

$$
\psi_{\varepsilon}(s):= \begin{cases}\frac{\theta}{2}(1+s) \ln \left[\frac{1+s}{2}\right]+\frac{\theta}{4 \varepsilon}(1-s)^{2}+\frac{\theta}{2}(1-s) \ln \left[\frac{\varepsilon}{2}\right]-\frac{\theta \varepsilon}{4} \text { if } s \geq 1-\varepsilon \\ \psi(s) & \text { if }|s| \leq 1-\varepsilon \\ \frac{\theta}{2}(1-s) \ln \left[\frac{1-s}{2}\right]+\frac{\theta}{4 \varepsilon}(1+s)^{2}+\frac{\theta}{2}(1+s) \ln \left[\frac{\varepsilon}{2}\right]-\frac{\theta \varepsilon}{4} \text { if } s \leq-1+\varepsilon\end{cases}
$$

The monotone function

$$
\phi_{\varepsilon}(s):=\psi_{\varepsilon}^{\prime}(s)= \begin{cases}\frac{\theta}{2}(1+\ln (1+s))-\frac{\theta}{2 \varepsilon}(1-s)-\frac{\theta}{2} \ln \varepsilon & \text { if } s \geq 1-\varepsilon \\ \phi(s) & \text { if }|s| \leq 1-\varepsilon \\ -\frac{\theta}{2}(1+\ln (1-s))+\frac{\theta}{2 \varepsilon}(1+s)+\frac{\theta}{2} \ln \varepsilon & \text { if } s \leq-1+\varepsilon\end{cases}
$$

has the following properties.

- For all $\varepsilon>0$

$$
\begin{array}{ll}
\phi(s) \geq \phi_{\varepsilon}(s) & \text { if } 1>s \geq 1-\varepsilon \\
\phi_{\varepsilon}(s) \geq \phi(s) & \text { if }-1+\varepsilon \geq s>-1
\end{array}
$$

- For all $r, s$

$$
\begin{aligned}
\Psi_{\varepsilon}^{\prime}(s)(r-s) & =\psi_{\varepsilon}^{\prime}(s)(r-s)-\theta_{c} s(r-s) \leq \psi_{\varepsilon}(r)-\psi_{\varepsilon}(s)+\theta_{c} s(s-r) \\
& =\Psi_{\varepsilon}(r)-\Psi_{\varepsilon}(s)+\frac{\theta_{c}}{2}(r-s)^{2},
\end{aligned}
$$

where we have used the identity

$$
2 s(s-r)=s^{2}-r^{2}+(s-r)^{2} \quad \forall r, s .
$$

- For $\varepsilon \leq \frac{1}{2}$ and for all $r, s$

$$
\theta(r-s)^{2} \leq\left(\phi_{\varepsilon}(r)-\phi_{\varepsilon}(s)\right)(r-s)
$$

and

$$
\begin{aligned}
\left(\phi_{\varepsilon}(r)-\phi_{\varepsilon}(s)\right)^{2} & \leq \phi_{\varepsilon}^{\prime}(\max \{|r|,|s|\})\left(\phi_{\varepsilon}(r)-\phi_{\varepsilon}(s)\right)(r-s) \\
& \leq \frac{\theta}{\varepsilon}\left(\phi_{\varepsilon}(r)-\phi_{\varepsilon}(s)\right)(r-s) .
\end{aligned}
$$

In addition, if $r, s>1-\varepsilon$ or $r, s<-1+\varepsilon$, then

$$
\frac{\theta}{2 \varepsilon}(r-s)^{2} \leq\left(\phi_{\varepsilon}(r)-\phi_{\varepsilon}(s)\right)(r-s) .
$$


Furthermore, it is a simple matter to show that $\Psi_{\varepsilon}$ is bounded below for $\varepsilon$ sufficiently small; e.g., if $\varepsilon \leq \varepsilon_{0}:=\theta /\left(8 \theta_{c}\right)$, then

$$
\Psi_{\varepsilon}(s) \geq \frac{\theta}{8 \varepsilon}\left([s-1]_{+}^{2}+[-1-s]_{+}^{2}\right)-\theta_{c} \geq-\theta_{c} \quad \forall s,
$$

where $[\cdot]_{+}:=\max \{\cdot, 0\}$. To see this, we note firstly for $|s| \leq 1$ that

$$
\Psi_{\varepsilon}(s) \geq \psi_{\varepsilon}(s) \geq \psi_{\varepsilon}(0) \equiv-\theta \ln 2 \geq-\theta_{c} .
$$

Secondly, for $s \geq 1$ we have under the stated assumption on $\varepsilon$ that

$$
\begin{aligned}
\Psi_{\varepsilon}(s) & \geq \frac{\theta}{4 \varepsilon}(s-1)^{2}-\frac{\theta \varepsilon}{4}+\frac{\theta_{c}}{2}\left(1-s^{2}\right) \\
& \equiv\left[\frac{\theta}{4 \varepsilon}-\frac{\theta_{c}}{2}\right](s-1)^{2}-\frac{\theta \varepsilon}{4}+\theta_{c}(1-s) \\
& \geq\left[\frac{\theta}{4 \varepsilon}-\theta_{c}\right](s-1)^{2}-\frac{\theta \varepsilon}{4}-\frac{\theta_{c}}{2},
\end{aligned}
$$

where we have applied a Young's inequality. Applying a similar bound for $s \leq-1$ yields the desired result (2.9).

For later purposes, we recall the following well-known Sobolev interpolation results, e.g., see [1]: let $p \in[1, \infty], m \geq 1$ and assume that $v \in W^{m, p}(\Omega)$. Then there are constants $C$ and $\mu=\frac{d}{m}\left(\frac{1}{p}-\frac{1}{r}\right)$ such that the inequality

$$
|v|_{0, r} \leq C|v|_{0, p}^{1-\mu}\|v\|_{m, p}^{\mu} \quad \text { holds for } r \in \begin{cases}{[p, \infty]} & \text { if } m-\frac{d}{p}>0 \\ {[p, \infty)} & \text { if } m-\frac{d}{p}=0 \\ {\left[p,-\frac{d}{m-(d / p)}\right]} & \text { if } m-\frac{d}{p}<0\end{cases}
$$

We now study the corresponding regularized version of $(\mathrm{P})$. $\left(\mathbf{P}_{\varepsilon}\right)$. Find $\left\{u_{\varepsilon}, w_{\varepsilon}\right\}$ such that $u_{\varepsilon}(\cdot, 0)=u_{0}(\cdot)$ and for a.e. $t \in(0, T)$

$$
\begin{gathered}
\left\langle\frac{\partial u_{\varepsilon}}{\partial t}, \eta\right\rangle+\left(b\left(u_{\varepsilon}\right) \nabla w_{\varepsilon}, \nabla \eta\right)=0 \quad \forall \eta \in H^{1}(\Omega), \\
\left(w_{\varepsilon}, \eta\right)=\gamma\left(\nabla u_{\varepsilon}, \nabla \eta\right)+\left(\Psi_{\varepsilon}^{\prime}\left(u_{\varepsilon}\right), \eta\right) \quad \forall \eta \in H^{1}(\Omega) .
\end{gathered}
$$

It is convenient to introduce the "inverse Laplacian" operator $\mathcal{G}: \mathcal{F} \rightarrow V$ such that

$$
(\nabla \mathcal{G} v, \nabla \eta)=\langle v, \eta\rangle \quad \forall \eta \in H^{1}(\Omega),
$$

where $\mathcal{F}:=\left\{v \in\left(H^{1}(\Omega)\right)^{\prime}:\langle v, 1\rangle=0\right\}$ and $V:=\left\{v \in H^{1}(\Omega):(v, 1)=0\right\}$. The well posedness of $\mathcal{G}$ follows from the Lax-Milgram theorem and the Poincaré inequality

$$
|\eta|_{0, p} \leq C\left(|\eta|_{1, p}+|(\eta, 1)|\right) \quad \forall \eta \in W^{1, p}(\Omega) \quad \text { and } \quad p \in[1, \infty] .
$$

One can define a norm on $\mathcal{F}$ by

$$
\|v\|_{-1}:=|\mathcal{G} v|_{1} \equiv\langle v, \mathcal{G} v\rangle^{\frac{1}{2}} \quad \forall v \in \mathcal{F} .
$$

We note also for future reference that using a Young's inequality yields for all $\alpha>0$ that

$(2.15)\langle v, \eta\rangle \equiv(\nabla \mathcal{G} v, \nabla \eta) \leq\|v\|_{-1}|\eta|_{1} \leq \frac{1}{2 \alpha}\|v\|_{-1}^{2}+\frac{\alpha}{2}|\eta|_{1}^{2} \quad \forall v \in \mathcal{F}, \eta \in H^{1}(\Omega)$.

In addition it follows from (2.12), (2.10) and (2.13) that

$$
|\nabla \mathcal{G} v|_{0} \leq C|v|_{0, r} \quad \forall v \in L^{r}(\Omega) \cap \mathcal{F},
$$

where $r=1,1+\tau, \frac{6}{5}$, for any $\tau>0$, for $d=1,2,3$, respectively. 
Assuming that $b_{\min }>0$ and given $q$ measurable in $\Omega$, it is also convenient to introduce the operator $\mathcal{G}_{q}: \mathcal{F} \rightarrow V$ such that

$$
\left(b(q) \nabla \mathcal{G}_{q} v, \nabla \eta\right)=\langle v, \eta\rangle \quad \forall \eta \in H^{1}(\Omega) .
$$

It follows for all $q$ measurable in $\Omega$ and $v \in \mathcal{F}$ that

$$
|\nabla \mathcal{G} v|_{0}^{2} \equiv\langle v, \mathcal{G} v\rangle \equiv\left(b(q) \nabla \mathcal{G}_{q} v, \nabla \mathcal{G} v\right) \leq b_{\max }^{\frac{1}{2}}\left|[b(q)]^{\frac{1}{2}} \nabla \mathcal{G}_{q} v\right|_{0}|\nabla \mathcal{G} v|_{0} .
$$

Similarly we have that

$$
\left|[b(q)]^{\frac{1}{2}} \nabla \mathcal{G}_{q} v\right|_{0}^{2} \equiv\left\langle v, \mathcal{G}_{q} v\right\rangle \equiv\left(\nabla \mathcal{G} v, \nabla \mathcal{G}_{q} v\right) \leq b_{\min }^{-\frac{1}{2}}|\nabla \mathcal{G} v|_{0}\left|[b(q)]^{\frac{1}{2}} \nabla \mathcal{G}_{q} v\right|_{0}
$$

Combining the above, it follows for all $q$ measurable in $\Omega$ and $v \in \mathcal{F}$ that

$$
b_{\min }\left|[b(q)]^{\frac{1}{2}} \nabla \mathcal{G}_{q} v\right|_{0}^{2} \leq|\nabla \mathcal{G} v|_{0}^{2} \leq b_{\max }\left|[b(q)]^{\frac{1}{2}} \nabla \mathcal{G}_{q} v\right|_{0}^{2} .
$$

Let $q$ be measurable in $\Omega, v \in \mathcal{F}$ and $\eta \in H^{1}(\Omega)$, then

$$
\langle v, \eta\rangle \equiv\left(b(q) \nabla \mathcal{G}_{q} v, \nabla \eta\right) \leq b_{\max }^{\frac{1}{2}}\left|[b(q)]^{\frac{1}{2}} \nabla \mathcal{G}_{q} v\right|_{0}|\eta|_{1}=b_{\max }^{\frac{1}{2}}\left\langle v, \mathcal{G}_{q} v\right\rangle^{\frac{1}{2}}|\eta|_{1}
$$

so that an analogue of (2.15) holds. Similarly to (2.16), we have from (2.17), (2.10) and (2.13) that

$$
\left|\nabla \mathcal{G}_{q} v\right|_{0} \leq C b_{\min }^{-1}|v|_{0, r} \quad \forall v \in L^{r}(\Omega) \cap \mathcal{F},
$$

where $r=1,1+\tau, \frac{6}{5}$, for any $\tau>0$, for $d=1,2,3$, respectively.

For a.e. $t \in(0, T)$, let $q(\cdot, t)$ be measurable in $\Omega$ and $v(\cdot, t) \in L^{2}(\Omega) \cap \mathcal{F}$ be such that $\frac{\partial q}{\partial t}(\cdot, t), \frac{\partial v}{\partial t}(\cdot, t) \in L^{2}(\Omega)$. If $b$ satisfies $(1.4 \mathrm{a}),(1.4 \mathrm{~b})$, then by differentiating (2.17) with respect to $t$ and setting $\eta \equiv \mathcal{G}_{q} v$ we obtain that

$$
\begin{aligned}
\left(\frac{\partial v}{\partial t}, \mathcal{G}_{q} v\right) & =\left(\frac{\partial}{\partial t}\left[b(q) \nabla \mathcal{G}_{q} v\right], \nabla \mathcal{G}_{q} v\right) \\
& =\left(b^{\prime}(q) \frac{\partial q}{\partial t},\left|\nabla \mathcal{G}_{q} v\right|^{2}\right)+\left(b(q) \nabla \frac{\partial}{\partial t}\left[\mathcal{G}_{q} v\right], \nabla \mathcal{G}_{q} v\right) \\
& =\left(b^{\prime}(q) \frac{\partial q}{\partial t},\left|\nabla \mathcal{G}_{q} v\right|^{2}\right)+\left(\frac{\partial}{\partial t}\left[\mathcal{G}_{q} v\right], v\right) .
\end{aligned}
$$

Hence applying (2.21) and noting (2.17) yields that

$$
\frac{\mathrm{d}}{\mathrm{dt}}\left(\mathcal{G}_{q} v, v\right)=\left(\frac{\partial}{\partial t}\left[\mathcal{G}_{q} v\right], v\right)+\left(\mathcal{G}_{q} v, \frac{\partial v}{\partial t}\right)=2\left(\mathcal{G}_{q} \frac{\partial v}{\partial t}, v\right)-\left(b^{\prime}(q) \frac{\partial q}{\partial t} \nabla \mathcal{G}_{q} v, \nabla \mathcal{G}_{q} v\right)
$$

We note for future reference that if $\frac{\partial q}{\partial t}(\cdot, t) \in L^{2}(\Omega)$ for a.e. $t \in(0, T)$ and if $b$ satisfies $(1.4 \mathrm{a}),(1.4 \mathrm{~b})$, then $(2.10)$ yields that

$$
\left|\left(b^{\prime}(q) \frac{\partial q}{\partial t} \nabla \mathcal{G}_{q} v, \nabla \mathcal{G}_{q} v\right)\right| \leq C\left|\frac{\partial q}{\partial t}\right|_{0}\left|\nabla \mathcal{G}_{q} v\right|_{0,4}^{2} \leq C\left|\frac{\partial q}{\partial t}\right|_{0}\left|\mathcal{G}_{q} v\right|_{1}^{2-\frac{d}{2}} \|\left.\mathcal{G}_{q} v\right|_{2} ^{\frac{d}{2}} .
$$

Similarly if $q_{1}, q_{2} \in H^{1}(\Omega)$ and $b$ satisfies (1.4a),(1.4b), then (2.10) yields that

$$
\begin{aligned}
& \left|\left[b\left(q_{2}\right)\right]^{\frac{1}{2}} \nabla\left(\mathcal{G}_{q_{1}}-\mathcal{G}_{q_{2}}\right) v\right|_{0} \equiv\left(\left[b\left(q_{2}\right)-b\left(q_{1}\right)\right] \nabla \mathcal{G}_{q_{1}} v, \nabla\left(\mathcal{G}_{q_{1}}-\mathcal{G}_{q_{2}}\right) v\right)^{\frac{1}{2}} \\
& \quad \leq \quad b_{\min }^{-\frac{1}{2}}\left|\left[b\left(q_{2}\right)-b\left(q_{1}\right)\right] \nabla \mathcal{G}_{q_{1}} v\right|_{0} \leq C b_{\min }^{-\frac{1}{2}}\left|q_{2}-q_{1}\right|_{0,4}\left|\nabla \mathcal{G}_{q_{1}} v\right|_{0,4} \\
& \quad \leq C b_{\min }^{-\frac{1}{2}}\left|q_{2}-q_{1}\right|_{0}^{1-\frac{d}{4}}\left\|q_{2}-q_{1}\right\|_{1}^{\frac{d}{4}}\left|\mathcal{G}_{q_{1}} v\right|_{1}^{1-\frac{d}{4}}\left\|\mathcal{G}_{q_{1}} v\right\|_{2}^{\frac{d}{4}} .
\end{aligned}
$$

Adapting an argument in [15], we now find a bound on $\left\|\mathcal{G}_{q} v\right\|_{2}$ when $b$ satisfies (1.4a),(1.4b), $q \in H^{2}(\Omega), v \in L^{2}(\Omega) \cap \mathcal{F}$ and $\Omega$ is a convex polyhedron or $\partial \Omega \in C^{1,1}$. It follows from the standard regularity estimate $|\cdot|_{2} \leq C|\Delta \cdot|_{0}, \mathcal{G}_{q} v \in V,(2.20)$, 
(2.17) and (2.10) with $r=\infty, \frac{2}{\tau}, 6, \mu=\frac{1}{2}, 1-\tau, 1$ and $s=2, \frac{2}{1-\tau}, 3, \lambda=0, \tau, \frac{1}{2}$ for all $\tau \in(0,1)$, when $d=1,2,3$, respectively, that

$$
\begin{aligned}
b_{\min }\left\|\mathcal{G}_{q} v\right\|_{2} & \leq C\left[b_{\min }\left|\Delta \mathcal{G}_{q} v\right|_{0}+|v|_{0}\right] \leq C\left[\left|b(q) \Delta \mathcal{G}_{q} v\right|_{0}+|v|_{0}\right] \\
& =C\left[\left|b^{\prime}(q) \nabla q . \nabla \mathcal{G}_{q} v+v\right|_{0}+|v|_{0}\right] \leq C\left[|\nabla q|_{0, r}\left|\nabla \mathcal{G}_{q} v\right|_{0, s}+|v|_{0}\right] \\
& \leq C\left[|q|_{1}^{1-\mu}\|q\|_{2}^{\mu}\left|\mathcal{G}_{q} v\right|_{1}^{1-\lambda}\left\|\mathcal{G}_{q} v\right\|_{2}^{\lambda}+|v|_{0}\right] .
\end{aligned}
$$

Finally using a Young's inequality yields that

$$
\left\|\mathcal{G}_{q} v\right\|_{2} \leq C_{b}\left[|q|_{1}^{\alpha}\|q\|_{2}^{\beta}\left|\mathcal{G}_{q} v\right|_{1}+|v|_{0}\right]
$$

where $\alpha=\frac{1}{2}, \frac{\tau}{1-\tau}, 0$, for all $\tau \in(0,1)$, and $\beta=2^{d-2}$ when $d=1,2,3$, respectively.

Choosing $\eta \equiv 1$ in (2.11a) yields that $\left\langle\frac{\partial u_{\varepsilon}}{\partial t}, 1\right\rangle=0$, i.e., $\left(u_{\varepsilon}(\cdot, t), 1\right)=\left(u_{0}(\cdot), 1\right)$ for all $t$. Hence it follows from (2.11a), (2.17), (1.4b) and (2.13) that

$$
w_{\varepsilon} \equiv-\mathcal{G}_{u_{\varepsilon}} \frac{\partial u_{\varepsilon}}{\partial t}+f \Psi_{\varepsilon}^{\prime}\left(u_{\varepsilon}\right)
$$

Therefore for $b_{\min }>0,\left(\mathrm{P}_{\varepsilon}\right)$ can be rewritten as follows.

Find $u_{\varepsilon}$ such that $u_{\varepsilon}(\cdot, 0)=u_{0}(\cdot)$ and for a.e. $t \in(0, T),\left(u_{\varepsilon}(\cdot, t), 1\right)=\left(u_{0}(\cdot), 1\right)$ and

$$
\gamma\left(\nabla u_{\varepsilon}, \nabla \eta\right)+\left(\Psi_{\varepsilon}^{\prime}\left(u_{\varepsilon}\right), \eta-f \eta\right)+\left(\mathcal{G}_{u_{\varepsilon}} \frac{\partial u_{\varepsilon}}{\partial t}, \eta\right)=0 \quad \forall \eta \in H^{1}(\Omega) .
$$

Similarly, if $b_{\min }>0(\mathrm{P})$ can be rewritten as follows.

Find $u$ such that $u(\cdot, 0)=u_{0}(\cdot)$ and for a.e. $t \in(0, T),(u(\cdot, t), 1)=\left(u_{0}(\cdot), 1\right)$ and

$$
\gamma(\nabla u, \nabla \eta)+\left(\Psi^{\prime}(u), \eta-f \eta\right)+\left(\mathcal{G}_{u} \frac{\partial u}{\partial t}, \eta\right)=0 \quad \forall \eta \in H^{1}(\Omega)
$$

with

$$
w \equiv-\mathcal{G}_{u} \frac{\partial u}{\partial t}+f \Psi^{\prime}(u) .
$$

Theorem 2.1. Let $d \leq 3$ and $u_{0} \in H^{1}(\Omega)$ be such that $\left\|u_{0}\right\|_{0, \infty} \leq 1$ and $\left|f u_{0}\right|<$ $1-\delta$ for some $\delta \in(0,1)$. If $b$ satisfies (1.4a) with $b_{\min }>0$, then for all $\varepsilon \leq$ $\varepsilon_{0}$ there exists $\left\{u_{\varepsilon}, w_{\varepsilon}\right\}$ solving $\left(\mathrm{P}_{\varepsilon}\right)$ such that the following stability bounds hold independently of $\varepsilon$

$$
\left\|u_{\varepsilon}\right\|_{L^{\infty}\left(0, T ; H^{1}(\Omega)\right)} \leq C, \quad\left\|u_{\varepsilon}\right\|_{H^{1}\left(0, T ;\left(H^{1}(\Omega)\right)^{\prime}\right)} \leq C
$$

and

$$
\left\|\left[u_{\varepsilon}-1\right]_{+}\right\|_{L^{\infty}\left(0, T ; L^{2}(\Omega)\right)}+\left\|\left[-u_{\varepsilon}-1\right]_{+}\right\|_{L^{\infty}\left(0, T ; L^{2}(\Omega)\right)} \leq C \varepsilon^{\frac{1}{2}} .
$$

In addition, the following stability bounds hold independently of $\varepsilon$ :

$$
\left\|w_{\varepsilon}\right\|_{L^{2}\left(0, T ; H^{1}(\Omega)\right)} \leq C_{b} \text { and }\left\|\phi_{\varepsilon}\left(u_{\varepsilon}\right)\right\|_{L^{2}\left(\Omega_{T}\right)} \leq C_{b} ;
$$

and if $\Omega$ is a convex polyhedron or $\partial \Omega \in C^{1,1}$,

$$
\left\|u_{\varepsilon}\right\|_{L^{2}\left(0, T ; H^{2}(\Omega)\right)} \leq C_{b} .
$$

Furthermore, if $b$ satisfies $(1.4 \mathrm{~b})$ and

$$
\begin{array}{cl}
\left\|\frac{\partial u_{\varepsilon}}{\partial t}\right\|_{L^{\frac{8}{8-d}}\left(0, T ; L^{2}(\Omega)\right)} \leq C\left(b_{\min }^{-1}, \varepsilon^{-1}\right), & \left\|u_{\varepsilon}\right\|_{L^{2^{d}}\left(0, T ; H^{2}(\Omega)\right)} \leq C\left(b_{\min }^{-1}, \varepsilon^{-1}\right), \\
\left\|w_{\varepsilon}\right\|_{L^{\frac{8}{6-d}}\left(0, T ; H^{1}(\Omega)\right)} \leq C\left(b_{\min }^{-1}, \varepsilon^{-1}\right),
\end{array}
$$

then the solution $\left\{u_{\varepsilon}, w_{\varepsilon}\right\}$ of $\left(\mathrm{P}_{\varepsilon}\right)$ is unique. 
Proof. Existence follows from standard arguments using Galerkin approximations and then passing to the limit; see [13]. The choices of $\eta$ below can be justified in a similar way. that

Choosing $\eta \equiv \frac{\partial u_{\varepsilon}}{\partial t}$ in $(2.28)$ and integrating over $(0, t)$ yields for all $t \in(0, T)$

$$
\begin{aligned}
\frac{\gamma}{2}\left|u_{\varepsilon}(\cdot, t)\right|_{1}^{2}+\left(\Psi_{\varepsilon}\left(u_{\varepsilon}(\cdot, t)\right), 1\right)+\int_{0}^{t} \mid\left[b\left(u_{\varepsilon}\right)\right]^{\frac{1}{2}} & \left.\nabla \mathcal{G}_{u_{\varepsilon}} \frac{\partial u_{\varepsilon}}{\partial s}(\cdot, s)\right|_{0} ^{2} \mathrm{~d} s \\
& =\frac{\gamma}{2}\left|u_{0}\right|_{1}^{2}+\left(\Psi_{\varepsilon}\left(u_{0}\right), 1\right) \leq C,
\end{aligned}
$$

where we have noted the assumptions on $u_{0}$. Hence the $\varepsilon$ independent bounds in (2.31) follow from noting (2.9), (2.13), (2.18) and (2.14). The bound (2.32) follows immediately from the bound on $\left(\Psi_{\varepsilon}\left(u_{\varepsilon}(\cdot, t)\right), 1\right)$ in $(2.36)$ and $(2.9)$.

Noting (2.27), (2.13) and (2.36) yields that

$$
\begin{aligned}
& \left\|(I-f) w_{\varepsilon}\right\|_{L^{2}\left(0, T ; H^{1}(\Omega)\right)} \\
& \quad \leq C\left\|\nabla \mathcal{G}_{u_{\varepsilon}} \frac{\partial u_{\varepsilon}}{\partial t}\right\|_{L^{2}\left(\Omega_{T}\right)} \leq C b_{\min }^{-\frac{1}{2}}\left\|\left[b\left(u_{\varepsilon}\right)\right]^{\frac{1}{2}} \nabla \mathcal{G}_{u_{\varepsilon}} \frac{\partial u_{\varepsilon}}{\partial t}\right\|_{L^{2}\left(\Omega_{T}\right)} \leq C b_{\min }^{-\frac{1}{2}} .
\end{aligned}
$$

Choosing $\eta \equiv \phi_{\varepsilon}\left(u_{\varepsilon}\right)$ in (2.28), noting that $\phi_{\varepsilon}^{\prime}(\cdot) \geq \theta$ and (2.13) yields for a.e. $t \in$ $(0, T)$ that

$$
\begin{aligned}
& 2 \gamma \theta\left|u_{\varepsilon}\right|_{1}^{2}+\left|(I-f) \phi_{\varepsilon}\left(u_{\varepsilon}\right)\right|_{0}^{2} \leq\left|\theta_{c} u_{\varepsilon}-\mathcal{G}_{u_{\varepsilon}} \frac{\partial u_{\varepsilon}}{\partial t}\right|_{0}^{2} \\
& \quad \leq C\left[\left|u_{\varepsilon}\right|_{0}^{2}+b_{\min }^{-1}\left|\left[b\left(u_{\varepsilon}\right)\right]^{\frac{1}{2}} \nabla \mathcal{G}_{u_{\varepsilon}} \frac{\partial u_{\varepsilon}}{\partial t}\right|_{0}^{2}\right] .
\end{aligned}
$$

Integrating the above over $t \in(0, T)$, noting (2.31) and (2.36) yields that

$$
\left\|(I-f) \phi_{\varepsilon}\left(u_{\varepsilon}\right)\right\|_{L^{2}\left(\Omega_{T}\right)} \leq C b_{\min }^{-\frac{1}{2}}
$$

Choosing $\eta \equiv u_{\varepsilon}$ in (2.28) yields for any constant $\lambda$ and for a.e. $t \in(0, T)$ that

$$
\begin{aligned}
& \left(\Psi_{\varepsilon}^{\prime}\left(u_{\varepsilon}\right), \lambda-f u_{\varepsilon}\right)=\left(\Psi_{\varepsilon}^{\prime}\left(u_{\varepsilon}\right), \lambda-u_{\varepsilon}\right)-\gamma\left|u_{\varepsilon}\right|_{1}^{2}-\left(\mathcal{G}_{u_{\varepsilon}} \frac{\partial u_{\varepsilon}}{\partial t}, u_{\varepsilon}\right) \\
& \quad \leq\left(\Psi_{\varepsilon}(\lambda)-\Psi_{\varepsilon}\left(u_{\varepsilon}\right), 1\right)+\frac{\theta_{c}}{2}\left|u_{\varepsilon}-\lambda\right|_{0}^{2}+C b_{\min }^{-\frac{1}{2}}\left|\left[b\left(u_{\varepsilon}\right)\right]^{\frac{1}{2}} \nabla \mathcal{G}_{u_{\varepsilon}} \frac{\partial u_{\varepsilon}}{\partial t}\right|_{0}\left|u_{\varepsilon}\right|_{0},
\end{aligned}
$$

where we have noted (2.4) and (2.13). Hence it follows on choosing $\lambda= \pm 1$ and noting (2.9) that

$$
\delta|\Omega|\left|f \Psi_{\varepsilon}^{\prime}\left(u_{\varepsilon}\right)\right| \leq C\left[1+\left|u_{\varepsilon}\right|_{0}^{2}+b_{\min }^{-\frac{1}{2}}\left|\left[b\left(u_{\varepsilon}\right)\right]^{\frac{1}{2}} \nabla \mathcal{G}_{u_{\varepsilon}} \frac{\partial u_{\varepsilon}}{\partial t}\right|_{0}\left|u_{\varepsilon}\right|_{0}\right] .
$$

Integrating the above over $t \in(0, T)$ and noting (2.31) and (2.36) yields that

$$
\left\|f \Psi_{\varepsilon}^{\prime}\left(u_{\varepsilon}\right)\right\|_{L^{2}\left(\Omega_{T}\right)} \leq C b_{\min }^{-\frac{1}{2}} .
$$

Combining (2.41), (2.37) and (2.39) yields the desired result (2.33). Finally (2.34) follows from (2.11b), (2.31), (2.33) and standard elliptic regularity theory.

Assuming that (2.28) has two solutions $u_{\varepsilon}^{1}, u_{\varepsilon}^{2}$ with corresponding $w_{\varepsilon}^{i}$ defined by (2.27), it follows that for a.e. $t \in(0, T) \bar{u}_{\varepsilon}:=u_{\varepsilon}^{1}-u_{\varepsilon}^{2} \in V$ satisfies

$$
\gamma\left|\bar{u}_{\varepsilon}\right|_{1}^{2}+\left(\phi_{\varepsilon}\left(u_{\varepsilon}^{1}\right)-\phi_{\varepsilon}\left(u_{\varepsilon}^{2}\right), \bar{u}_{\varepsilon}\right)+\left(\mathcal{G}_{u_{\varepsilon}^{1}} \frac{\partial u_{\varepsilon}^{1}}{\partial t}-\mathcal{G}_{u_{\varepsilon}} \frac{\partial u_{\varepsilon}^{2}}{\partial t}, \bar{u}_{\varepsilon}\right)=\theta_{c}\left|\bar{u}_{\varepsilon}\right|_{0}^{2} .
$$


If $b$ satisfies (1.4a),(1.4b), on noting (2.22), (2.23), (2.24), (2.26), (2.27), (2.19), (2.18), and applying Hölders inequality, it follows that for a.e. $t \in(0, T)$

$$
\begin{aligned}
\gamma\left|\bar{u}_{\varepsilon}\right|_{1}^{2}+ & \left(\phi_{\varepsilon}\left(u_{\varepsilon}^{1}\right)-\phi_{\varepsilon}\left(u_{\varepsilon}^{2}\right), \bar{u}_{\varepsilon}\right)+\frac{1}{2} \frac{\mathrm{d}}{\mathrm{d} t}\left(\mathcal{G}_{u_{\varepsilon}^{1}} \bar{u}_{\varepsilon}, \bar{u}_{\varepsilon}\right) \\
= & \theta_{c}\left|\bar{u}_{\varepsilon}\right|_{0}^{2}-\frac{1}{2}\left(b^{\prime}\left(u_{\varepsilon}^{1}\right) \frac{\partial u_{\varepsilon}^{1}}{\partial t} \nabla \mathcal{G}_{u_{\varepsilon}^{1}} \bar{u}_{\varepsilon}, \nabla \mathcal{G}_{u_{\varepsilon}^{1}} \bar{u}_{\varepsilon}\right)-\left(\left(\mathcal{G}_{u_{\varepsilon}^{1}}-\mathcal{G}_{u_{\varepsilon}^{2}}\right) \frac{\partial u_{\varepsilon}^{2}}{\partial t}, \bar{u}_{\varepsilon}\right) \\
\leq & \theta_{c}\left|\bar{u}_{\varepsilon}\right|_{0}^{2}+C\left|\frac{\partial u_{\varepsilon}^{1}}{\partial t}\right|_{0}\left|\mathcal{G}_{u_{\varepsilon}^{1}} \bar{u}_{\varepsilon}\right|_{1}^{2-\frac{d}{2}} \|\left.\mathcal{G}_{u_{\varepsilon}^{1}} \bar{u}_{\varepsilon}\right|_{2} ^{\frac{d}{2}} \\
& +\left.C_{b}\left|\bar{u}_{\varepsilon}\right|_{0}^{1-\frac{d}{4}}\left\|\left.\bar{u}_{\varepsilon}\right|_{1} ^{\frac{d}{4}}\left|\mathcal{G}_{u_{\varepsilon}^{1}} \bar{u}_{\varepsilon}\right|_{1}^{1-\frac{d}{4}}\right\| \mathcal{G}_{u_{\varepsilon}^{1}} \bar{u}_{\varepsilon}\right|_{2} ^{\frac{d}{4}}\left|w_{\varepsilon}^{2}\right|_{1} \\
\leq & \frac{\gamma}{2}\left|\bar{u}_{\varepsilon}\right|_{1}^{2}+C_{b}\left(\mathcal{G}_{u_{\varepsilon}^{1}} \bar{u}_{\varepsilon}, \bar{u}_{\varepsilon}\right)\left[1+\left|\frac{\partial u_{\varepsilon}^{1}}{\partial t}\right|_{0}^{\frac{8}{8-d}}+\left|\frac{\partial u_{\varepsilon}^{1}}{\partial t}\right|_{0}\left|u_{\varepsilon}^{1}\right|_{1}^{\frac{\alpha d}{2}} \|\left. u_{\varepsilon}^{1}\right|_{2} ^{\frac{\beta d}{2}}\right. \\
& \left.\quad+\left|u_{\varepsilon}^{1}\right|_{1}^{\frac{4 \alpha d}{12-d}} \|\left. u_{\varepsilon}^{1}\right|_{2} ^{\frac{4 \beta d}{12-d}}\left|w_{\varepsilon}^{2}\right|_{1}^{\frac{16}{12-d}}+\left|w_{\varepsilon}^{2}\right|_{1}^{\frac{8}{6-d}}\right] \\
\leq & \frac{\gamma}{2}\left|\bar{u}_{\varepsilon}\right|_{1}^{2}+C_{b}\left(\mathcal{G}_{u_{\varepsilon}^{1}} \bar{u}_{\varepsilon}, \bar{u}_{\varepsilon}\right)\left[1+\left|\frac{\partial u_{\varepsilon}^{1}}{\partial t}\right|_{0}^{\frac{8}{8-d}}+\left.\left|u_{\varepsilon}^{1}\right|\right|_{1} ^{4 \alpha}\left\|u_{\varepsilon}^{1}\right\|_{2}^{4 \beta}+\left|w_{\varepsilon}^{2}\right|_{1}^{\frac{8}{6-d}}\right]
\end{aligned}
$$

Uniqueness then follows from noting (2.6), (2.31), (2.35), a Gronwall inequality, (2.13) and (2.27).

We note that the integral assumption on the initial data, in Theorem 2.1 above, only excludes the physically uninteresting case of $u_{0} \equiv \pm 1$, when only one component is present.

Remark. If $\frac{\partial q}{\partial t}(\cdot, t) \in V$, for a.e. $t \in(0, T)$, then alternatively to (2.23), we have from (2.15) and (2.16) that

$$
\begin{aligned}
& \left|\left(b^{\prime}(q) \frac{\partial q}{\partial t},\left|\nabla \mathcal{G}_{q} v\right|^{2}\right)\right| \\
& \quad \leq\left|\frac{\partial q}{\partial t}\right|_{1}\left|\mathcal{G}\left[(I-f)\left(b^{\prime}(q)\left|\nabla \mathcal{G}_{q} v\right|^{2}\right)\right]\right|_{1} \leq C\left|\frac{\partial q}{\partial t}\right|_{1}\left|\mathcal{G}_{q} v\right|_{1,2(1+\tau)}^{2},
\end{aligned}
$$

where $\tau=0$, any $\tau>0, \tau=\frac{1}{5}$, for $d=1,2,3$, respectively. Using this inequality, when performing a similar computation to that in (2.43), yields uniqueness of a solution to $\left(\mathrm{P}_{\varepsilon}\right)$ with the first assumption in (2.35) replaced by

$$
\left\|\frac{\partial u_{\varepsilon}}{\partial t}\right\|_{L^{\nu}\left(0, T ; H^{1}(\Omega)\right)} \leq C\left(b_{\min }^{-1}, \varepsilon^{-1}\right) \quad \nu=1,1+\frac{\tau}{2+\tau}, \frac{8}{7} \quad \text { for } d=1,2,3 .
$$

Corollary 2.1. Let $u_{0} \in H^{3}(\Omega), \frac{\partial u_{0}}{\partial \nu}=0$ on $\partial \Omega$ and $\delta \in(0,1)$ be such that $\left\|u_{0}\right\|_{0, \infty} \leq 1-\delta$. Let $d \leq 3$ with either $\Omega$ being a convex polyhedron or $\partial \Omega \in C^{1,1}$. Let $b$ satisfy $(1.4 \mathrm{a}),(1.4 \mathrm{~b})$. Then for all $\varepsilon \leq \varepsilon_{0}(\delta)$ solutions $\left\{u_{\varepsilon}, w_{\varepsilon}\right\}$ of $\left(P_{\varepsilon}\right)$ are such that the following stability bounds hold independently of $\varepsilon$ :

$$
\begin{gathered}
\left\|\frac{\partial u_{\varepsilon}}{\partial t}\right\|_{L^{2}\left(0, T_{\star} ; H^{1}(\Omega)\right)}+\left\|\frac{\partial u_{\varepsilon}}{\partial t}\right\|_{L^{\infty}\left(0, T_{\star} ;\left(H^{1}(\Omega)\right)^{\prime}\right)}+\left\|w_{\varepsilon}\right\|_{L^{\infty}\left(0, T_{\star} ; H^{1}(\Omega)\right)} \leq C_{b}, \\
\left\|\phi_{\varepsilon}\left(u_{\varepsilon}\right)\right\|_{L^{\infty}\left(0, T_{\star} ; L^{2}(\Omega)\right)}+\left\|u_{\varepsilon}\right\|_{L^{\infty}\left(0, T_{\star} ; H^{2}(\Omega)\right)}+\left\|w_{\varepsilon}\right\|_{L^{2}\left(0, T_{\star} ; H^{2}(\Omega)\right)} \leq C_{b},
\end{gathered}
$$

where $T_{\star}=T$ if $d \leq 2$, and $T_{\star} \in(0, T]$ if $d=3$. Hence the solution $\left\{u_{\varepsilon}, w_{\varepsilon}\right\}$ of $\left(\mathrm{P}_{\varepsilon}\right)$ is unique over $\Omega_{T_{\star}}$.

Proof. Differentiating (2.11b) with respect to $t$ and setting $\eta=\frac{\partial u_{\varepsilon}}{\partial t}$, noting $\phi_{\varepsilon}^{\prime}(r) \geq$ $\theta \geq 0,(2.11 \mathrm{a}),(2.27),(2.21)$ and $(2.22)$ yields that for a.e. $t \in\left(0, T_{\star}\right)$

$$
\begin{aligned}
& \gamma\left|\frac{\partial u_{\varepsilon}}{\partial t}\right|_{1}^{2}-\theta_{c}\left|\frac{\partial u_{\varepsilon}}{\partial t}\right|_{0}^{2} \leq \gamma\left|\frac{\partial u_{\varepsilon}}{\partial t}\right|_{1}^{2}+\left(\phi_{\varepsilon}^{\prime}\left(u_{\varepsilon}\right) \frac{\partial u_{\varepsilon}}{\partial t}, \frac{\partial u_{\varepsilon}}{\partial t}\right)-\theta_{c}\left|\frac{\partial u_{\varepsilon}}{\partial t}\right|_{0}^{2}=\left\langle\frac{\partial w_{\varepsilon}}{\partial t}, \frac{\partial u_{\varepsilon}}{\partial t}\right\rangle \\
& \quad=-\left(b\left(u_{\varepsilon}\right) \nabla \frac{\partial w_{\varepsilon}}{\partial t}, \nabla w_{\varepsilon}\right)=-\frac{1}{2} \frac{\mathrm{d}}{\mathrm{d} t}\left[\left(b\left(u_{\varepsilon}\right) \nabla w_{\varepsilon}, \nabla w_{\varepsilon}\right)\right]+\frac{1}{2}\left(b^{\prime}\left(u_{\varepsilon}\right) \frac{\partial u_{\varepsilon}}{\partial t},\left|\nabla w_{\varepsilon}\right|^{2}\right),
\end{aligned}
$$


where $T_{\star} \in(0, T]$. Once again this differentiation and these choices of test function can be justified in the standard way by using a Galerkin approximation and then passing to the limit. Noting (2.44), the following inequality holds for $\tau=0$, any $\tau \in\left(0, \frac{1}{5}\right], \tau=\frac{1}{5}$ for $d=1,2,3$, respectively

$$
\left|\left(b^{\prime}\left(u_{\varepsilon}\right) \frac{\partial u_{\varepsilon}}{\partial t},\left|\nabla w_{\varepsilon}\right|^{2}\right)\right| \leq \frac{\gamma}{2}\left|\frac{\partial u_{\varepsilon}}{\partial t}\right|_{1}^{2}+C\left|w_{\varepsilon}\right|_{1,2(1+\tau)}^{4} .
$$

Next we note from (2.10), (2.27) and (2.26) that

$$
\begin{aligned}
\left|w_{\varepsilon}\right|_{1,2(1+\tau)}^{4} & \leq C_{b}\left|w_{\varepsilon}\right|_{1}^{4(1-\mu)}\left\|\mathcal{G}_{u_{\varepsilon}} \frac{\partial u_{\varepsilon}}{\partial t}\right\|_{2}^{4 \mu} \\
& \leq C_{b}\left[\left|w_{\varepsilon}\right|_{1}^{4}\left|u_{\varepsilon}\right|_{1}^{4 \mu \alpha}\left\|u_{\varepsilon}\right\|_{2}^{4 \mu \beta}+\left|w_{\varepsilon}\right|_{1}^{4(1-\mu)}\left|\frac{\partial u_{\varepsilon}}{\partial t}\right|_{0}^{4 \mu}\right] \\
& \leq C_{b}\left[\left|w_{\varepsilon}\right|_{1}^{4}\left|u_{\varepsilon}\right|_{1}^{4 \mu \alpha}\left\|u_{\varepsilon}\right\|_{2}^{4 \mu \beta}+\left|w_{\varepsilon}\right|_{1}^{\frac{4(1-\mu)}{1-2 \mu}}+\left|\frac{\partial u_{\varepsilon}}{\partial t}\right|_{0}^{2}\right],
\end{aligned}
$$

where $\mu=\frac{d \tau}{2(1+\tau)}$. It follows from standard elliptic regularity, (2.38), (2.40) and (2.27) that

$$
\left\|u_{\varepsilon}\right\|_{2} \leq C\left[\left\|u_{\varepsilon}\right\|_{1}+\left|\phi_{\varepsilon}\left(u_{\varepsilon}\right)\right|_{0}+\left|w_{\varepsilon}\right|_{0}\right] \leq C_{b}\left[1+\left|u_{\varepsilon}\right|_{0}\right]\left[1+\left|u_{\varepsilon}\right|_{0}+\left|w_{\varepsilon}\right|_{1}\right] .
$$

Combining (2.47), (2.48), (2.49), (2.50) and noting (2.19) and (2.27) yields that for a.e. $t \in\left(0, T_{\star}\right)$

$$
\begin{gathered}
\gamma\left|\frac{\partial u_{\varepsilon}}{\partial t}\right|_{1}^{2}+\frac{1}{2} \frac{\mathrm{d}}{\mathrm{d} t}\left[\left(b\left(u_{\varepsilon}\right) \nabla w_{\varepsilon}, \nabla w_{\varepsilon}\right)\right] \leq C_{b}\left|\frac{\partial u_{\varepsilon}}{\partial t}\right|_{0}^{2}+C\left(b_{\min }^{-1},\left\|u_{\varepsilon}\right\|_{1}\right)\left[1+\left|w_{\varepsilon}\right|_{1}^{4(1+\rho)}\right] \\
\leq C\left(b_{\min }^{-1},\left\|u_{\varepsilon}\right\|_{1}\right)\left[1+\left(b\left(u_{\varepsilon}\right) \nabla w_{\varepsilon}, \nabla w_{\varepsilon}\right)^{2(1+\rho)}\right]
\end{gathered}
$$

where $\rho=\frac{5 \tau}{2}$. We set $B(t):=\max \left\{\left(b\left(u_{\varepsilon}(\cdot, t)\right) \nabla w_{\varepsilon}(\cdot, t), \nabla w_{\varepsilon}(\cdot, t)\right), 1\right\}$ for a.e. $t \in(0, T)$. It follows from (2.33) that

$$
\int_{0}^{T} B(t) \mathrm{d} t \leq C\left(b_{\min }^{-1}, T\right)
$$

From (2.51) and the above notation we have for a.e. $t \in\left(0, T_{\star}\right)$ that

$$
\frac{\mathrm{d} B}{\mathrm{~d} t} \leq C\left(b_{\min }^{-1},\left\|u_{\varepsilon}\right\|_{1}\right) B^{2(1+\rho)} .
$$

Via a Galerkin approximation, one can show in the standard way that

$$
\begin{aligned}
B(0) & \leq C\left[1+\left|w_{\varepsilon}(0)\right|_{1}^{2}\right] \\
& \leq C\left[1+\left|\Delta u_{0}\right|_{1}^{2}+\left\|\Psi_{\varepsilon}^{\prime \prime}\left(u_{0}\right)\right\|_{0, \infty}^{2}\left|u_{0}\right|_{1}^{2}\right] \leq C\left[1+\left\|u_{0}\right\|_{3}^{2}\right] \leq C,
\end{aligned}
$$

provided $u_{0} \in H^{3}(\Omega), \frac{\partial u_{0}}{\partial \nu}=0$ on $\partial \Omega$ and $\varepsilon_{0} \leq \delta$; see for example Proposition 2.5 in [5].

For $d=1$, i.e., $\rho=0$, it follows from (2.53) and (2.52) that for a.e. $t \in(0, T)$

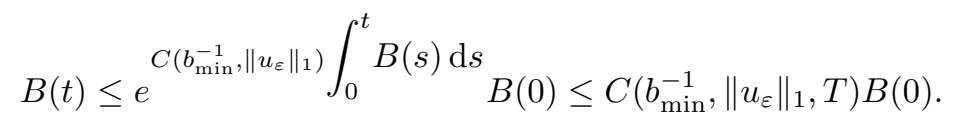

For $d=2$, i.e., for any $\rho \in\left(0, \frac{1}{2}\right)$, it follows from (2.53) that for a.e. $t \in\left(0, T_{\star}\right)$

$$
-\frac{1}{2 \rho} \frac{\mathrm{d}}{\mathrm{d} t} B^{-2 \rho} \leq C\left(b_{\min }^{-1},\left\|u_{\varepsilon}\right\|_{1}\right) B .
$$


Hence we have from (2.56) and (2.52) that

$$
\begin{aligned}
B(t) & \leq\left[1-2 \rho C\left(b_{\min }^{-1},\left\|u_{\varepsilon}\right\|_{1}\right)[B(0)]^{2 \rho} \int_{0}^{t} B(s) \mathrm{d} s\right]^{-\frac{1}{2 \rho}} B(0) \\
& \leq\left[1+4 \rho C\left(b_{\min }^{-1},\left\|u_{\varepsilon}\right\|_{1}\right)[B(0)]^{2 \rho} \int_{0}^{t} B(s) \mathrm{d} s\right]^{\frac{1}{2 \rho}} B(0) \\
& \leq e^{C\left(b_{\min }^{-1},\left\|u_{\varepsilon}\right\|_{1}\right)[B(0)]^{2 \rho} \int_{0}^{t} B(s) \mathrm{d} s} B(0) \\
& \leq C\left(b_{\min }^{-1},\left\|u_{\varepsilon}\right\|_{1}, T, B(0)\right) \quad t \in(0, T),
\end{aligned}
$$

provided $\rho \in\left(0, \frac{1}{2}\right)$ is chosen sufficiently small so that

$$
4 \rho C\left(b_{\min }^{-1},\left\|u_{\varepsilon}\right\|_{1}\right)[B(0)]^{2 \rho} \int_{0}^{T} B(s) \mathrm{d} s \leq 1 .
$$

For $d=3$, i.e., $\rho=\frac{1}{5}$, it follows from (2.53) that for a.e. $t \in\left(0, T_{\star}\right)$

$$
-\frac{1}{1+2 \rho} \frac{\mathrm{d}}{\mathrm{d} t} B^{-(1+2 \rho)} \leq C\left(b_{\min }^{-1},\left\|u_{\varepsilon}\right\|_{1}\right)
$$

Hence we have from (2.59) that

$$
\begin{aligned}
B(t) & \leq\left[1-(1+2 \rho) C\left(b_{\min }^{-1},\left\|u_{\varepsilon}\right\|_{1}\right)[B(0)]^{1+2 \rho} t\right]^{-\frac{1}{(1+2 \rho)}} B(0) \\
& \leq\left[1+2(1+2 \rho) C\left(b_{\min }^{-1},\left\|u_{\varepsilon}\right\|_{1}\right)[B(0)]^{1+2 \rho} t\right]^{\frac{1}{1+2 \rho}} B(0) \\
& \leq e^{C\left(b_{\min }^{-1},\left\|u_{\varepsilon}\right\|_{1}\right)[B(0)]^{1+2 \rho} t} B(0) \\
& \leq C\left(b_{\min }^{-1},\left\|u_{\varepsilon}\right\|_{1}, T, B(0)\right) \quad t \in\left(0, T_{\star}\right),
\end{aligned}
$$

provided $T_{\star}$ is such that

$$
2(1+2 \rho) C\left(b_{\min }^{-1},\left\|u_{\varepsilon}\right\|_{1}\right)[B(0)]^{1+2 \rho} T_{\star} \leq 1 .
$$

The third bound in (2.46a) then follows from (2.55), (2.57), (2.60), (2.54), (2.31), (2.27), (2.13) and (2.40). The first bound in (2.46a) then follows from the third and (2.51). Similarly, the second follows from the third on noting (2.27) and (2.18). The first two bounds in (2.46b) follow from the third in (2.46a) and (2.50). The third bound in (2.46b) follows from the first two in (2.46b) on noting (2.27), (2.26), (2.46a) and (2.13). Finally, uniqueness of a solution to $\left(\mathrm{P}_{\varepsilon}\right)$ over $\Omega_{T_{\star}}$ follows from the bounds (2.46a), (2.46b) and Theorem 2.1.

Theorem 2.2. If $d \leq 3, b$ satisfies (1.4a) with $b_{\min }>0$ and the assumptions on $u_{0}$ of Theorem 2.1 hold, then there exists $\{u, w\}$ solving $(\mathrm{P})$ such that

$$
\begin{gathered}
u \in L^{\infty}\left(0, T ; H^{1}(\Omega)\right) \cap H^{1}\left(0, T ;\left(H^{1}(\Omega)\right)^{\prime}\right), \\
w \in L^{2}\left(0, T ; H^{1}(\Omega)\right) \text { and } \phi(u) \in L^{2}\left(\Omega_{T}\right) ;
\end{gathered}
$$

and if either $\Omega$ is a convex polyhedron or $\partial \Omega \in C^{1,1}$

$$
u \in L^{2}\left(0, T ; H^{2}(\Omega)\right) \text {. }
$$

Furthermore, if the assumptions on $u_{0}$ and $b$ of Corollary 2.1 hold, then the solution $\{u, w\}$ of $(\mathrm{P})$ is such that

$$
u \in L^{\infty}\left(0, T_{\star} ; H^{2}(\Omega)\right) \cap H^{1}\left(0, T_{\star} ; H^{1}(\Omega)\right) \cap W^{1, \infty}\left(0, T_{\star} ;\left(H^{1}(\Omega)\right)^{\prime}\right),
$$

$$
w \in L^{\infty}\left(0, T_{\star} ; H^{1}(\Omega)\right) \cap L^{2}\left(0, T_{\star} ; H^{2}(\Omega)\right) \text { and } \phi(u) \in L^{\infty}\left(0, T_{\star} ; L^{2}(\Omega)\right) \text {, }
$$


where $T_{\star}=T$ if $d \leq 2$, and $T_{\star} \in(0, T]$ if $d=3$. Moreover, the solution $\{u, w\}$ of $(\mathrm{P})$ is unique over $\Omega_{T_{\star}}$ and we have that

$$
\left\|u-u_{\varepsilon}\right\|_{L^{2}\left(0, T_{\star} ; H^{1}(\Omega)\right)}^{2}+\left\|u-u_{\varepsilon}\right\|_{L^{\infty}\left(0, T_{\star} ;\left(H^{1}(\Omega)\right)^{\prime}\right)}^{2} \leq C_{b} \varepsilon .
$$

Proof. As the bounds (2.31) and (2.33) are independent of $\varepsilon$, it follows that there exists $u \in L^{\infty}\left(0, T ; H^{1}(\Omega)\right) \cap H^{1}\left(0, T ;\left(H^{1}(\Omega)\right)^{\prime}\right), \phi^{\star} \in L^{2}\left(\Omega_{T}\right)$ and a subsequence $\left\{u_{\varepsilon^{\prime}}\right\}$ such that as $\varepsilon^{\prime} \rightarrow 0$

$$
u_{\varepsilon^{\prime}} \rightarrow u \text { in } L^{\infty}\left(0, T ; H^{1}(\Omega)\right) \text { weak-star and in } H^{1}\left(0, T ;\left(H^{1}(\Omega)\right)^{\prime}\right) \text { weakly, }
$$

$$
w_{\varepsilon^{\prime}} \rightarrow w \quad \text { in } L^{2}\left(0, T ; H^{1}(\Omega)\right) \text { weakly } \quad \text { and } \quad \phi_{\varepsilon^{\prime}}\left(u_{\varepsilon^{\prime}}\right) \rightarrow \phi^{\star} \quad \text { in } L^{2}\left(\Omega_{T}\right) \text { weakly. }
$$

Using an argument from [2], it follows that $\phi^{\star} \equiv \phi(u)$. Therefore taking the limit $\varepsilon^{\prime} \rightarrow 0$ in $(2.11 \mathrm{~b})$ yields that $\{u, w\}$ solves $(1.5 \mathrm{~b})$. It is implied by $(2.68)$ that $u_{\varepsilon^{\prime}} \rightarrow u$ in $L^{2}\left(\Omega_{T}\right)$ strongly as $\varepsilon^{\prime} \rightarrow 0$; see [18]. Noting this, (1.4a) and (2.69), it follows for a.e. $t \in(0, T)$ that as $\varepsilon^{\prime} \rightarrow 0$

$$
\left(b\left(u_{\varepsilon^{\prime}}\right) \nabla w_{\varepsilon^{\prime}}, \nabla \eta\right) \rightarrow(b(u) \nabla w, \nabla \eta) \quad \forall \eta \in W^{1, \infty}(\Omega) .
$$

Therefore taking the limit $\varepsilon^{\prime} \rightarrow 0$ in (2.11a) yields, on noting the above and (2.68), that $\{u, w\}$ solves (1.5a). Hence we have existence of a solution $\{u, w\}$, satisfying (2.62) and (2.63), of (P). The regularity results (2.64), (2.65) and (2.66) follow similarly to the $\varepsilon$ independent bounds $(2.34),(2.46 \mathrm{a}),(2.46 \mathrm{~b})$. Uniqueness of a solution to $(\mathrm{P})$ over $\Omega_{T_{\star}}$ then follows as for $\left(\mathrm{P}_{\varepsilon}\right)$; see (2.42) and (2.43).

We now prove an error bound between the unique solutions $u$ and $u_{\varepsilon}$ of problems (P) and $\left(\mathrm{P}_{\varepsilon}\right)$. Set $e:=u-u_{\varepsilon}$. Subtraction of (2.28) from (2.29), noting (2.22) and choosing $\eta=e$ yields for a.e. $t \in\left(0, T_{\star}\right)$ that

$$
\begin{aligned}
& \gamma|e|_{1}^{2}+\left(\phi(u)-\phi_{\varepsilon}\left(u_{\varepsilon}\right), e\right)+\frac{1}{2} \frac{\mathrm{d}}{\mathrm{d} t}\left(\mathcal{G}_{u} e, e\right) \\
& \quad=\theta_{c}|e|_{0}^{2}-\frac{1}{2}\left(b^{\prime}(u) \frac{\partial u}{\partial t} \nabla \mathcal{G}_{u} e, \nabla \mathcal{G}_{u} e\right)-\left(\left(\mathcal{G}_{u}-\mathcal{G}_{u_{\varepsilon}}\right) \frac{\partial u_{\varepsilon}}{\partial t}, e\right) .
\end{aligned}
$$

From the monotonicity of $\phi_{\varepsilon}$ and (2.8) it follows that for a.e. $t \in\left(0, T_{\star}\right)$

$$
\left(\phi_{\varepsilon}(u)-\phi_{\varepsilon}\left(u_{\varepsilon}\right), e\right) \geq \frac{\theta}{2 \varepsilon} \int_{\Omega_{\varepsilon}^{+}(t) \cup \Omega_{\varepsilon}^{-}(t)} e^{2} \mathrm{~d} x,
$$

where

$$
\begin{array}{r}
\Omega_{\varepsilon}^{+}(t):=\left\{x \in \Omega: 1-\varepsilon \leq u(x, t) \leq u_{\varepsilon}(x, t)\right\}, \\
\Omega_{\varepsilon}^{-}(t):=\left\{x \in \Omega: u_{\varepsilon}(x, t) \leq u(x, t) \leq-1+\varepsilon\right\} .
\end{array}
$$

Next we note from the definition of $\phi_{\varepsilon}$ and (2.3) that

1. If $|r| \leq 1-\varepsilon$, then $\phi_{\varepsilon}(r) \equiv \phi(r)$.

2. If $r \geq 1-\varepsilon$ and $s \leq r$, then $\left(\phi_{\varepsilon}(r)-\phi(r)\right)(r-s) \leq 0$.

3. If $r \leq-1+\varepsilon$ and $r \leq s$, then $\left(\phi_{\varepsilon}(r)-\phi(r)\right)(r-s) \leq 0$.

Hence it follows for a.e $t \in\left(0, T_{\star}\right)$ that

$$
\left(\phi_{\varepsilon}(u)-\phi(u), e\right) \leq \int_{\Omega_{\varepsilon}^{+}(t) \cup \Omega_{\varepsilon}^{-}(t)}\left(\phi_{\varepsilon}(u)-\phi(u)\right) e \mathrm{~d} x \leq-\int_{\Omega_{\varepsilon}^{+}(t) \cup \Omega_{\varepsilon}^{-}(t)} \phi(u) e \mathrm{~d} x .
$$


Substituting (2.71) and (2.72) into (2.70), and as (1.4a),(1.4b) hold it follows similarly to (2.43) on noting $(2.23),(2.24),(2.26),(2.27),(2.19)$ and (2.18) that for a.e. $t \in\left(0, T_{\star}\right)$

$$
\begin{aligned}
\gamma|e|_{1}^{2}+ & \frac{\theta}{2 \varepsilon} \int_{\Omega_{\varepsilon}^{+}(t) \cup \Omega_{\varepsilon}^{-}(t)} e^{2} \mathrm{~d} x+\frac{1}{2} \frac{\mathrm{d}}{\mathrm{d} t}\left(\mathcal{G}_{u} e, e\right) \\
\leq & \left(\phi_{\varepsilon}(u)-\phi(u), e\right)+\theta_{c}|e|_{0}^{2}-\frac{1}{2}\left(b^{\prime}(u) \frac{\partial u}{\partial t} \nabla \mathcal{G}_{u} e, \nabla \mathcal{G}_{u} e\right)-\left(\left(\mathcal{G}_{u}-\mathcal{G}_{u_{\varepsilon}}\right) \frac{\partial u_{\varepsilon}}{\partial t}, e\right) \\
\leq & -\int_{\Omega_{\varepsilon}^{+}(t) \cup \Omega_{\varepsilon}^{-}(t)} \phi(u) e \mathrm{~d} x+\theta_{c}|e|_{0}^{2}+C\left|\frac{\partial u}{\partial t}\right|_{0}\left|\mathcal{G}_{u} e\right|_{1}^{2-\frac{d}{2}}\left\|\mathcal{G}_{u} e\right\|_{2}^{\frac{d}{2}} \\
& +C_{b}|e|_{0}^{1-\frac{d}{4}}\|e\|_{1}^{\frac{d}{4}}\left|\mathcal{G}_{u} e\right|_{1}^{1-\frac{d}{4}}\left\|\mathcal{G}_{u} e\right\|_{2}^{\frac{d}{4}}\left|w_{\varepsilon}\right|_{1} \\
\leq & C \varepsilon \int_{\Omega_{\varepsilon}^{+}(t) \cup \Omega_{\varepsilon}^{-}(t)}[\phi(u)]^{2} \mathrm{~d} x \\
& +C_{b}\left(\mathcal{G}_{u} e, e\right)\left[1+\left|\frac{\partial u}{\partial t}\right|_{0}^{\frac{8}{8-d}}+|u|_{1}^{4 \alpha}\|u\|_{2}^{4 \beta}+\left|w_{\varepsilon}\right|_{1}^{\frac{8}{6-d}}\right] .
\end{aligned}
$$

The desired result (2.67) then follows from noting (2.46a), (2.65), (2.66), a Gronwall inequality, (2.13), (2.17), (2.18) and (2.14).

Remark. We note that the assumption $b_{\min }>0$ in Theorems 2.1 and 2.2 can be relaxed in order to establish existence of a solution $\left\{u_{\varepsilon}, w_{\varepsilon}\right\}$ to $\left(\mathrm{P}_{\varepsilon}\right)$, with the bounds (2.31) and (2.32) holding, and a solution $\{u, w\}$ to (P), with (2.62) holding; see [13].

\section{Finite element approximation}

Throughout the rest of the paper we assume that the assumptions (A) hold. We now consider the following fully discrete approximation to $\left(\mathrm{P}_{\varepsilon}\right)$; which is a regularized version of $\left(\mathrm{P}^{h, \Delta t}\right)$, see Section 1 .

$\left(\mathbf{P}_{\varepsilon}^{h, \Delta t}\right)$. For $n \geq 1$, find $\left\{U_{\varepsilon}^{n}, W_{\varepsilon}^{n}\right\} \in S^{h} \times S^{h}$ such that

$$
\begin{gathered}
\left(\frac{U_{\varepsilon}^{n}-U_{\varepsilon}^{n-1}}{\Delta t}, \chi\right)^{h}+b_{\max }\left(\nabla W_{\varepsilon}^{n}, \nabla \chi\right) \\
=\left(\left[b_{\max }-b\left(U_{\varepsilon}^{n-1}\right)\right] \nabla W_{\varepsilon}^{n-1}, \nabla \chi\right) \quad \forall \chi \in S^{h}, \\
\left(W_{\varepsilon}^{n}, \chi\right)^{h}=\gamma\left(\nabla U_{\varepsilon}^{n}, \nabla \chi\right)+\left(\phi_{\varepsilon}\left(U_{\varepsilon}^{n}\right)-\theta_{c} U_{\varepsilon}^{n}, \chi\right)^{h} \quad \forall \chi \in S^{h},
\end{gathered}
$$

where $U_{\varepsilon}^{0} \equiv Q^{h} u_{0}$ or $\hat{Q}^{h} u_{0}$ and $W_{\varepsilon}^{0} \in S^{h}$ is such that

$$
\left(W_{\varepsilon}^{0}, \chi\right)^{h}=-\gamma\left(\Delta u_{0}, \chi\right)+\left(\phi_{\varepsilon}\left(U_{\varepsilon}^{0}\right)-\theta_{c} U_{\varepsilon}^{0}, \chi\right)^{h} \quad \forall \chi \in S^{h} .
$$

Similarly to (2.12), we introduce the operator $\mathcal{G}^{h}: \mathcal{F} \rightarrow V^{h}$ such that

$$
\left(\nabla \mathcal{G}^{h} v, \nabla \chi\right)=\langle v, \chi\rangle \quad \forall \chi \in S^{h},
$$

where $V^{h}:=\left\{v^{h} \in S^{h}:\left(v^{h}, 1\right)=0\right\}$. We have the following analogues of (2.14) and (2.15). We define a norm on $\mathcal{F}$ by

$$
\|v\|_{-h}:=\left|\mathcal{G}^{h} v\right|_{1}=\left\langle v, \mathcal{G}^{h} v\right\rangle^{\frac{1}{2}} \quad \forall v \in \mathcal{F},
$$

and for $\alpha>0$ we have that

$$
\langle v, \chi\rangle \equiv\left(\nabla \mathcal{G}^{h} v, \nabla \chi\right) \leq\|v\|_{-h}|\chi|_{1} \leq \frac{1}{2 \alpha}\|v\|_{-h}^{2}+\frac{\alpha}{2}|\chi|_{1}^{2} \quad \forall v \in \mathcal{F}, \chi \in S^{h}
$$


Below we recall some well-known results concerning $S^{h}$ : for $m=0$ or 1 , and for $p \geq 2$

$$
\begin{aligned}
& (3.5) \quad\left|\left(I-\pi^{h}\right) \eta\right|_{m, p} \leq C h^{2-m-d\left(\frac{1}{2}-\frac{1}{p}\right)}|\eta|_{2} \quad \forall \eta \in H^{2}(\Omega) ; \\
& (3.6) \quad\left|\left(I-Q^{h}\right) \eta\right|_{0}+h\left|\left(I-Q^{h}\right) \eta\right|_{1} \leq C h^{m}|\eta|_{m} \quad \forall \eta \in H^{m}(\Omega), m=1 \text { or } 2 ; \\
& (3.7) \quad|\chi|_{0}^{2} \leq|\chi|_{h}^{2}:=(\chi, \chi)^{h} \leq(d+2)|\chi|_{0}^{2} \quad \forall \chi \in S^{h} ; \\
& (3.8)\left|\left(\chi_{1}, \chi_{2}\right)-\left(\chi_{1}, \chi_{2}\right)^{h}\right| \leq C h^{1+m}\left\|\chi_{1}\right\|_{m}\left\|\chi_{2}\right\|_{1} \quad \forall \chi_{1}, \chi_{2} \in S^{h}, m=0 \text { or } 1 ; \\
& \text { (3.9) } \quad\left|\left(\mathcal{G}-\mathcal{G}^{h}\right) v\right|_{0} \leq C h^{2-m}\|v\|_{-m}, \quad \forall v \in\left(H^{m}(\Omega)\right)^{\prime} \cap \mathcal{F}, m=0 \text { or } 1 .
\end{aligned}
$$

Next we note that

$$
C_{1} h^{2}\left|v^{h}\right|_{1} \leq C_{2} h\left|v^{h}\right|_{0} \leq\left\|v^{h}\right\|_{-h} \leq\left\|v^{h}\right\|_{-1} \leq C_{3}\left\|v^{h}\right\|_{-h} \quad \forall v^{h} \in V^{h} .
$$

The first inequality on the left is just an inverse inequality, recalling that the partitioning is quasi-uniform, and holds for any $v^{h} \in S^{h}$. The second bound follows from the first and (3.4). The third follows from noting that $\left|\mathcal{G}^{h} v^{h}\right|_{1} \leq\left|\mathcal{G} v^{h}\right|_{1}$. The final inequality follows from noting (3.9) with $m=0$ and the second inequality above. In addition, we note the inverse inequality for $1 \leq p_{1} \leq p_{2} \leq \infty$ and $m=0$ or 1

$$
|\chi|_{m, p_{2}} \leq C h^{\frac{d\left(p_{1}-p_{2}\right)}{p_{1} p_{2}}}|\chi|_{m, p_{1}} \quad \forall \chi \in S^{h} .
$$

The following bounds concerning $Q^{h}$ and $\hat{Q}^{h}$ are also easily established. From (3.6), (3.5) and (3.11) we have that

$$
\left|\left(I-Q^{h}\right) \eta\right|_{0, \infty} \leq C h^{2-\frac{d}{2}}|\eta|_{2} \quad \forall \eta \in H^{2}(\Omega) .
$$

Comparing $\hat{Q}^{h} \eta$ with $Q^{h} \eta$ and noting (3.8) and the first inequality on the left of (3.10) yields that

$$
\left|\left(I-\hat{Q}^{h}\right) \eta\right|_{0}+h\left|\left(I-\hat{Q}^{h}\right) \eta\right|_{1} \leq C h|\eta|_{1} \quad \forall \eta \in H^{1}(\Omega) .
$$

Since $\left(\hat{Q}^{h} \eta\right)\left(x_{j}\right) \equiv\left(\eta, \chi_{j}\right) /\left(1, \chi_{j}\right) \quad j=1 \rightarrow J$; where $\chi_{j} \in S^{h}$ and $\chi_{j}\left(x_{i}\right)=\delta_{i j}$, it follows that

$$
\left\|\hat{Q}^{h} \eta\right\|_{0, \infty} \leq\|\eta\|_{0, \infty} \quad \forall \eta \in L^{\infty}(\Omega) .
$$

Furthermore for a uniform mesh if $d=1$ and for a uniform translation invariant mesh if $d=2$ and $\Omega$ is a rectangle, we note from (1.6) and the above that

$$
\begin{aligned}
& \left|\left(\pi^{h}-\hat{Q}^{h}\right) \eta\right|_{h}^{2} \leq C h^{-d} \sum_{j=1}^{J}\left[\left(\eta, \chi_{j}\right)-\left(\eta, \chi_{j}\right)^{h}\right]^{2} \\
& \quad \leq C h^{-d} \sum_{j \in J_{B}}\left[\left(\eta, \chi_{j}\right)-\left(\eta, \chi_{j}\right)^{h}\right]^{2}+C h^{-d} \sum_{j \in J_{I}}\left[\left(\eta, \chi_{j}\right)-\left(\eta, \chi_{j}\right)^{h}\right]^{2} \\
& \quad \leq C h^{3}|\eta|_{1, \infty}^{2}+C h^{4}|\eta|_{2, \infty}^{2} \leq C h^{3}\|\eta\|_{2, \infty}^{2} \quad \forall \eta \in W^{2, \infty}(\Omega),
\end{aligned}
$$

where $J_{B}:=\left\{j: x_{j} \in \partial \Omega\right\}, J_{I}:=\left\{j: x_{j} \notin \partial \Omega\right\}$ and we have noted that $\# J_{B} \leq C h^{1-d}, \# J_{I} \leq C h^{-d}$.

Since $\phi_{\varepsilon}$ is monotone it follows (see [12, p. 68]) that

$$
\left|\left(I-\pi^{h}\right)\left[\phi_{\varepsilon}(\chi)\right]\right|_{0} \leq C h\left|\pi^{h}\left[\phi_{\varepsilon}(\chi)\right]\right|_{1} \quad \forall \chi \in S^{h} .
$$

Furthermore, as the partitioning is (weakly) acute, it follows from (2.7) for $\varepsilon \leq \frac{1}{2}$ that $\forall \chi \in S^{h}$

$$
\left|\nabla \pi^{h}\left[\phi_{\varepsilon}(\chi)\right]\right|_{0}^{2} \leq \phi_{\varepsilon}^{\prime}\left(\|\chi\|_{0, \infty}\right)\left(\nabla \chi, \nabla \pi^{h}\left[\phi_{\varepsilon}(\chi)\right]\right) \leq \frac{\theta}{\varepsilon}\left(\nabla \chi, \nabla \pi^{h}\left[\phi_{\varepsilon}(\chi)\right]\right) ;
$$

see $[9]$ and $[19, \S 2.4 .2]$. 
In addition to $(3.2)$ we introduce $\hat{\mathcal{G}}^{h}: \mathcal{F}^{h} \rightarrow V^{h}$, defined by

$$
\left(\nabla \hat{\mathcal{G}}^{h} v, \nabla \chi\right)=(v, \chi)^{h} \quad \forall \chi \in S^{h},
$$

where $\mathcal{F}^{h}:=\left\{v \in C(\bar{\Omega}):(v, 1)^{h}=0\right\}$. Note that $V^{h} \subset \mathcal{F}^{h}$ and the analogue of (3.4) holds: for $\alpha>0$

$$
(v, \chi)^{h} \equiv\left(\nabla \hat{\mathcal{G}}^{h} v, \nabla \chi\right) \leq\left|\hat{\mathcal{G}}^{h} v\right|_{1}|\chi|_{1} \leq \frac{1}{2 \alpha}\left|\hat{\mathcal{G}}^{h} v\right|_{1}^{2}+\frac{\alpha}{2}|\chi|_{1}^{2} \quad \forall v \in \mathcal{F}^{h}, \chi \in S^{h} .
$$

It is easily deduced from (3.8) (e.g., see [6]) that

$$
\left\|\left(\mathcal{G}^{h}-\hat{\mathcal{G}}^{h}\right) v^{h}\right\|_{1} \leq C h^{2}\left\|v^{h}\right\|_{1}, \quad \forall v^{h} \in V^{h} .
$$

In addition, we have the analogue of (3.10)

$$
h^{2}\left|v^{h}\right|_{1} \leq C_{1} h\left|v^{h}\right|_{h} \leq C_{2}\left|\hat{\mathcal{G}}^{h} v^{h}\right|_{1} \leq C_{3}\left|\mathcal{G}^{h} v^{h}\right|_{1} \leq C_{4}\left|\hat{\mathcal{G}}^{h} v^{h}\right|_{1} \quad \forall v^{h} \in V^{h} .
$$

The first inequality on the left is just an inverse inequality on noting (3.7) and holds for all $v^{h} \in S^{h}$. The second follows from the first and (3.19). The third and fourth follow from (3.20) and noting the first two inequalities in (3.10) and (3.21), respectively.

It follows from (3.1a) with $\chi \equiv 1$ and (1.7) that

$$
\left(U_{\varepsilon}^{n}, 1\right)=\left(U_{\varepsilon}^{0}, 1\right)=\left(u_{0}, 1\right) \quad n \geq 1 .
$$

Then similarly to $(2.28),\left(\mathrm{P}_{\varepsilon}^{h, \Delta t}\right)$ can be rewritten as follows.

For $n \geq 1$ find $U_{\varepsilon}^{n} \in S^{h}$ such that $\left(U_{\varepsilon}^{n}, 1\right)=\left(u_{0}, 1\right)$ and

$$
\begin{aligned}
\gamma\left(\nabla U_{\varepsilon}^{n}, \nabla \chi\right) & +\left(\phi_{\varepsilon}\left(U_{\varepsilon}^{n}\right)-\theta_{c} U_{\varepsilon}^{n},(I-f) \chi\right)^{h}+b_{\max }^{-1}\left(\hat{\mathcal{G}}^{h}\left(\frac{U_{\varepsilon}^{n}-U_{\varepsilon}^{n-1}}{\Delta t}\right), \chi\right)^{h} \\
& =\left(W_{\varepsilon}^{n-1}-b_{\max }^{-1} \hat{\mathcal{G}}^{h} J_{\varepsilon}^{n-1},(I-f) \chi\right)^{h} \quad \forall \chi \in S^{h}
\end{aligned}
$$

where $J_{\varepsilon}^{n-1} \in V^{h}$ is such that

$$
\left(J_{\varepsilon}^{n-1}, \chi\right)^{h}=\left(b\left(U_{\varepsilon}^{n-1}\right) \nabla W_{\varepsilon}^{n-1}, \nabla \chi\right) \quad \forall \chi \in S^{h},
$$$$
\text { where } U_{\varepsilon}^{0} \equiv Q^{h} u_{0} \text { or } \hat{Q}^{h} u_{0} \text { and } W_{\varepsilon}^{0} \in S^{h} \text { satisfies (3.1c). }
$$

We note for future reference that from (3.24) and (3.18) it follows that

$$
\left|\hat{\mathcal{G}}^{h} J_{\varepsilon}^{n}\right|_{1} \leq C\left|W_{\varepsilon}^{n}\right|_{1} \quad n \geq 0 .
$$

Lemma 3.1. Let $d \leq 3$ and the assumptions on $u_{0}$ of Theorem 1.1 and the assumptions (A) hold. If $b$ satisfies (1.4a), and $\Delta t \leq 4 \gamma /\left[b_{\max } \theta_{c}^{2}\right]$, then for all $\varepsilon \leq \varepsilon_{0}(\delta)$ and for all $h>0$ such that

$$
\left\|U_{\varepsilon}^{0}\right\|_{0, \infty} \leq 1-\frac{1}{2} \delta
$$

there exists a unique solution $\left\{U_{\varepsilon}^{n}, W_{\varepsilon}^{n}\right\}_{n=0}^{N}$ to $\left(\mathrm{P}_{\varepsilon}^{h, \Delta t}\right)$ satisfying

$$
\begin{aligned}
& \max _{n=0 \rightarrow N}\left|U_{\varepsilon}^{n}\right|_{1}^{2}+b_{\min }^{2} \sum_{n=1}^{N}\left|U_{\varepsilon}^{n}-U_{\varepsilon}^{n-1}\right|_{1}^{2}+\left|W_{\varepsilon}^{0}\right|_{1}^{2}+b_{\min } \Delta t \sum_{n=1}^{N}\left|W_{\varepsilon}^{n}\right|_{1}^{2} \\
& \quad+b_{\min } \Delta t \sum_{n=1}^{N}\left[\left|\hat{\mathcal{G}}^{h}\left(\frac{U_{\varepsilon}^{n}-U_{\varepsilon}^{n-1}}{\Delta t}\right)\right|_{1}^{2}+\varepsilon\left|\pi^{h}\left[\phi_{\varepsilon}\left(U_{\varepsilon}^{n}\right)\right]\right|_{1}^{2}+\left|\pi^{h}\left[\phi_{\varepsilon}\left(U_{\varepsilon}^{n}\right)\right]\right|_{0}^{2}\right] \leq C .
\end{aligned}
$$


Furthermore, we have that

$$
\left|\left[U_{\varepsilon}^{n}-1\right]_{+}\right|_{h}+\left|\left[-U_{\varepsilon}^{n}-1\right]_{+}\right|_{h} \leq C \varepsilon^{\frac{1}{2}} \quad n=1 \rightarrow N .
$$

Proof. As $U_{\varepsilon}^{0} \equiv Q^{h} u_{0}$ or $\hat{Q}^{h} u_{0}$, existence and uniqueness of $U_{\varepsilon}^{0}$ follows immediately. Moreover, from (3.6), (3.13) and the assumption (3.27) it follows that

$$
\left|U_{\varepsilon}^{0}\right|_{1}^{2}+\left(\Psi_{\varepsilon}\left(U_{\varepsilon}^{0}\right), 1\right)^{h} \leq C .
$$

Existence and uniqueness of $W_{\varepsilon}^{0}$ follows immediately from (3.1c). Furthermore, we have that

$$
W_{\varepsilon}^{0} \equiv-\hat{Q}^{h}\left(\gamma \Delta u_{0}\right)+\pi^{h}\left[\phi_{\varepsilon}\left(U_{\varepsilon}^{0}\right)\right]-\theta_{c} U_{\varepsilon}^{0} .
$$

Hence we have from (3.31), (3.13), (3.17), (3.30) and (3.27) and the assumptions on $u_{0}$ that for $\varepsilon \leq \varepsilon_{0}(\delta) \leq \frac{\delta}{2}$

$$
\left\|W_{\varepsilon}^{0}\right\|_{1} \leq C\left[\left\|u_{0}\right\|_{3}+\left\|U_{\varepsilon}^{0}\right\|_{1}\right] \leq C .
$$

Existence of $U_{\varepsilon}^{n}$ follows by noting that for fixed $n \geq 1,(3.23)$ is the EulerLagrange equation of the minimization problem

$$
\begin{aligned}
\min _{\chi \in K^{h}} \frac{\gamma}{2}|\chi|_{1}^{2} & +\left(\Psi_{\varepsilon}(\chi), 1\right)^{h} \\
& +\frac{1}{2 b_{\max } \Delta t}\left|\nabla \hat{\mathcal{G}}^{h}\left(\chi-U_{\varepsilon}^{n-1}\right)\right|_{0}^{2}-\left(W_{\varepsilon}^{n-1}-b_{\max }^{-1} \hat{\mathcal{G}}^{h} J_{\varepsilon}^{n-1}, \chi\right)^{h},
\end{aligned}
$$

where $K^{h}:=\left\{\chi \in S^{h}:(\chi, 1)=\left(u_{0}, 1\right)\right\}$. For fixed $n \geq 1$, if (3.23) has two solutions $U_{\varepsilon}^{n, 1}$ and $U_{\varepsilon}^{n, 2}$, then $\bar{U}_{\varepsilon}^{n}:=U_{\varepsilon}^{n, 1}-U_{\varepsilon}^{n, 2} \in V^{h}$ satisfies

$$
\gamma\left|\bar{U}_{\varepsilon}^{n}\right|_{1}^{2}+\left(\phi_{\varepsilon}\left(U_{\varepsilon}^{n, 1}\right)-\phi_{\varepsilon}\left(U_{\varepsilon}^{n, 2}\right), \bar{U}_{\varepsilon}^{n}\right)^{h}+\frac{1}{b_{\max } \Delta t}\left|\hat{\mathcal{G}}^{h} \bar{U}_{\varepsilon}^{n}\right|_{1}^{2}=\theta_{c}\left|\bar{U}_{\varepsilon}^{n}\right|_{h}^{2} .
$$

It follows from (2.6) and (3.19) with $\alpha=\frac{1}{2} b_{\max } \theta_{c} \Delta t$ that

$$
\gamma\left|\bar{U}_{\varepsilon}^{n}\right|_{1}^{2}+\theta\left|\bar{U}_{\varepsilon}^{n}\right|_{h}^{2}+\frac{1}{b_{\max } \Delta t}\left|\hat{\mathcal{G}}^{h} \bar{U}_{\varepsilon}^{n}\right|_{1}^{2} \leq \theta_{c}\left|\bar{U}_{\varepsilon}^{n}\right|_{h}^{2} \leq \frac{1}{b_{\max } \Delta t}\left|\hat{\mathcal{G}}^{h} \bar{U}_{\varepsilon}^{n}\right|_{1}^{2}+\frac{b_{\max } \theta_{c}^{2} \Delta t}{4}\left|\bar{U}_{\varepsilon}^{n}\right|_{1}^{2}
$$

from which uniqueness of $U_{\varepsilon}^{n}$ follows under the stated condition on $\Delta t$. Existence and uniqueness of $W_{\varepsilon}^{n}, n \geq 1$, follows immediately from (3.1b).

We now prove the stability bound (3.28). For fixed $n \geq 1$ choosing $\chi \equiv W_{\varepsilon}^{n}$ in (3.1a), $\chi \equiv\left(U_{\varepsilon}^{n}-U_{\varepsilon}^{n-1}\right) / \Delta t$ in (3.1b) and combining yields that

$$
\gamma\left(\nabla U_{\varepsilon}^{n}, \nabla\left(U_{\varepsilon}^{n}-U_{\varepsilon}^{n-1}\right)\right)+\left(\phi_{\varepsilon}\left(U_{\varepsilon}^{n}\right)-\theta_{c} U_{\varepsilon}^{n}, U_{\varepsilon}^{n}-U_{\varepsilon}^{n-1}\right)^{h}+b_{\max } \Delta t\left|W_{\varepsilon}^{n}\right|_{1}^{2}
$$

$$
=\Delta t\left(\left[b_{\max }-b\left(U_{\varepsilon}^{n-1}\right)\right] \nabla W_{\varepsilon}^{n-1}, \nabla W_{\varepsilon}^{n}\right) .
$$

Noting (2.4) and the identity (2.5) yields that

$$
\begin{aligned}
\frac{\gamma}{2}\left|U_{\varepsilon}^{n}\right|_{1}^{2} & +\left(\Psi_{\varepsilon}\left(U_{\varepsilon}^{n}\right), 1\right)^{h}+\frac{\gamma}{2}\left|U_{\varepsilon}^{n}-U_{\varepsilon}^{n-1}\right|_{1}^{2} \\
& +\frac{\Delta t}{2}\left\{b_{\max }\left|W_{\varepsilon}^{n}\right|_{1}^{2}+\left|\left[b\left(U_{\varepsilon}^{n-1}\right)\right]^{\frac{1}{2}} \nabla W_{\varepsilon}^{n}\right|_{0}^{2}\right. \\
& \left.+\left|\left[b_{\max }-b\left(U_{\varepsilon}^{n-1}\right)\right]^{\frac{1}{2}} \nabla\left(W_{\varepsilon}^{n}-W_{\varepsilon}^{n-1}\right)\right|_{0}^{2}\right\} \\
\leq & \frac{\gamma}{2}\left|U_{\varepsilon}^{n-1}\right|_{1}^{2}+\left(\Psi_{\varepsilon}\left(U_{\varepsilon}^{n-1}\right), 1\right)^{h}+\frac{\theta_{c}}{2}\left|U_{\varepsilon}^{n}-U_{\varepsilon}^{n-1}\right|_{h}^{2} \\
& +\frac{\Delta t}{2}\left|\left[b_{\max }-b\left(U_{\varepsilon}^{n-1}\right)\right]^{\frac{1}{2}} \nabla W_{\varepsilon}^{n-1}\right|_{0}^{2} .
\end{aligned}
$$


Choosing $\chi \equiv \Delta t\left(U_{\varepsilon}^{n}-U_{\varepsilon}^{n-1}\right)$ in (3.1a) and applying Young's inequalities yields that

$$
\begin{aligned}
\frac{\theta_{c}}{2} \mid & U_{\varepsilon}^{n}-\left.U_{\varepsilon}^{n-1}\right|_{h} ^{2} \\
= & -\frac{\theta_{c} \Delta t}{2}\left(\left[b_{\max }-b\left(U_{\varepsilon}^{n-1}\right)\right] \nabla\left(W_{\varepsilon}^{n}-W_{\varepsilon}^{n-1}\right)+b\left(U_{\varepsilon}^{n-1}\right) \nabla W_{\varepsilon}^{n}, \nabla\left(U_{\varepsilon}^{n}-U_{\varepsilon}^{n-1}\right)\right) \\
\leq & \frac{\Delta t}{2}\left|\left[b_{\max }-b\left(U_{\varepsilon}^{n-1}\right)\right]^{\frac{1}{2}} \nabla\left(W_{\varepsilon}^{n}-W_{\varepsilon}^{n-1}\right)\right|_{0}^{2}+\frac{\left(b_{\min }+2 b_{\max }\right) \Delta t}{4 b_{\max }}\left|\left[b\left(U_{\varepsilon}^{n-1}\right)\right]^{\frac{1}{2}} \nabla W_{\varepsilon}^{n}\right|_{0}^{2} \\
\quad & \quad \frac{\theta_{c}^{2} \Delta t}{8}\left|\left[b_{\max }-b\left(U_{\varepsilon}^{n-1}\right)\right]^{\frac{1}{2}} \nabla\left(U_{\varepsilon}^{n}-U_{\varepsilon}^{n-1}\right)\right|_{0}^{2} \\
& +\frac{b_{\max } \theta_{c}^{2} \Delta t}{4\left(b_{\min }+2 b_{\max }\right)}\left|\left[b\left(U_{\varepsilon}^{n-1}\right)\right]^{\frac{1}{2}} \nabla\left(U_{\varepsilon}^{n}-U_{\varepsilon}^{n-1}\right)\right|_{0}^{2} \\
\leq & \frac{\Delta t}{2}\left|\left[b_{\max }-b\left(U_{\varepsilon}^{n-1}\right)\right]^{\frac{1}{2}} \nabla\left(W_{\varepsilon}^{n}-W_{\varepsilon}^{n-1}\right)\right|_{0}^{2}+\frac{\Delta t}{2}\left|\left[b\left(U_{\varepsilon}^{n-1}\right)\right]^{\frac{1}{2}} \nabla W_{\varepsilon}^{n}\right|_{0}^{2} \\
(3.35) & +\frac{b_{\min } \Delta t}{4}\left|W_{\varepsilon}^{n}\right|_{1}^{2}+\frac{b_{\max } \theta_{c}^{2} \Delta t}{8}\left[1-\frac{b_{\mathrm{var}}^{2}}{2+b_{\mathrm{var}}}\right]\left|U_{\varepsilon}^{n}-U_{\varepsilon}^{n-1}\right|_{1}^{2},
\end{aligned}
$$

where $b_{\text {var }}:=b_{\min } / b_{\max }$. Combining (3.34) and (3.35) under the stated condition on $\Delta t$ yields for $n \geq 1$ that

$$
\begin{gathered}
\frac{\gamma}{2}\left|U_{\varepsilon}^{n}\right|_{1}^{2}+\frac{\gamma b_{\text {var }}^{2}}{2\left(2+b_{\text {var }}\right)}\left|U_{\varepsilon}^{n}-U_{\varepsilon}^{n-1}\right|_{1}^{2}+\left(\Psi_{\varepsilon}\left(U_{\varepsilon}^{n}\right), 1\right)^{h}+\frac{\left(2 b_{\max }-b_{\min }\right) \Delta t}{4}\left|W_{\varepsilon}^{n}\right|_{1}^{2} \\
\leq \frac{\gamma}{2}\left|U_{\varepsilon}^{n-1}\right|_{1}^{2}+\left(\Psi_{\varepsilon}\left(U_{\varepsilon}^{n-1}\right), 1\right)^{h}+\frac{\left(b_{\max }-b_{\min }\right) \Delta t}{2}\left|W_{\varepsilon}^{n-1}\right|_{1}^{2} .
\end{gathered}
$$

Summing (3.36) from $n=1 \rightarrow m$ yields for all $m \leq N$ that

$$
\begin{gathered}
\frac{\gamma}{2}\left|U_{\varepsilon}^{m}\right|_{1}^{2}+\frac{\gamma b_{\mathrm{var}}^{2}}{2\left(2+b_{\mathrm{var}}\right)} \sum_{n=1}^{m}\left|U_{\varepsilon}^{n}-U_{\varepsilon}^{n-1}\right|_{1}^{2}+\left(\Psi_{\varepsilon}\left(U_{\varepsilon}^{m}\right), 1\right)^{h}+\frac{b_{\min } \Delta t}{4} \sum_{n=1}^{m}\left|W_{\varepsilon}^{n}\right|_{1}^{2} \\
\leq \frac{\gamma}{2}\left|U_{\varepsilon}^{0}\right|_{1}^{2}+\left(\Psi_{\varepsilon}\left(U_{\varepsilon}^{0}\right), 1\right)^{h}+\frac{\left(b_{\max }-b_{\min }\right) \Delta t}{2}\left|W_{\varepsilon}^{0}\right|_{1}^{2} \leq C,
\end{gathered}
$$

where we have noted (3.30) and (3.32). Hence the first four bounds of (3.28) hold. The fifth bound of (3.28) holds on noting (3.25) and (3.26). Furthermore, the bound (3.29) follows immediately from the bounds on $\left(\Psi_{\varepsilon}\left(U_{\varepsilon}^{n}\right), 1\right)^{h}, n=1 \rightarrow N$, above and $(2.9)$.

Choosing $\chi \equiv \pi^{h}\left[\phi_{\varepsilon}\left(U_{\varepsilon}^{n}\right)\right]$ in (3.23), summing from $n=1 \rightarrow N$ and noting the bounds above and (3.17) yields, similarly to (2.38) and (2.39), that

$$
b_{\min } \Delta t \sum_{n=1}^{N}\left[\varepsilon\left|\pi^{h}\left[\phi_{\varepsilon}\left(U_{\varepsilon}^{n}\right)\right]\right|_{1}^{2}+\left|(I-f) \pi^{h}\left[\phi_{\varepsilon}\left(U_{\varepsilon}^{n}\right)\right]\right|_{h}^{2}\right] \leq C .
$$

Choosing $\chi \equiv U_{\varepsilon}^{n}$ in (3.23) and rearranging, similarly to (2.40), yields for any constant $\lambda$

$$
\begin{aligned}
\left(\phi_{\varepsilon}\left(U_{\varepsilon}^{n}\right), \lambda-f U_{\varepsilon}^{n}\right)^{h} \leq & \left(\psi_{\varepsilon}(\lambda)-\psi_{\varepsilon}\left(U_{\varepsilon}^{n}\right), 1\right)^{h}-b_{\max }^{-1}\left(\hat{\mathcal{G}}^{h}\left(\frac{U_{\varepsilon}^{n}-U_{\varepsilon}^{n-1}}{\Delta t}\right), U_{\varepsilon}^{n}\right)^{h} \\
& +\left(\theta_{c} U_{\varepsilon}^{n}+W_{\varepsilon}^{n-1}-b_{\max }^{-1} \hat{\mathcal{G}}^{h} J_{\varepsilon}^{n-1},(I-f) U_{\varepsilon}^{n}\right)^{h}
\end{aligned}
$$

Choosing $\lambda= \pm 1$ in (3.39) and summing $n=1 \rightarrow N$ yields, similarly to (2.41), that

$$
b_{\min } \Delta t \sum_{n=1}^{N}\left|f \pi^{h}\left[\phi_{\varepsilon}\left(U_{\varepsilon}^{n}\right)\right]\right|_{h}^{2} \leq C .
$$

Combining (3.38), (3.40) and the first bound in (3.28) and noting (3.7) yields the remaining bounds in (3.28). 
Lemma 3.2. Let $d \leq 2$ and the assumptions on $u_{0}$ of Theorem 1.1 and the assumptions (A) hold. Let $b$ satisfy (1.4a),(1.4b) and $\Delta t \leq 4 \gamma /\left[b_{\max } \theta_{c}^{2}\right]$. Then for all $\varepsilon \leq \varepsilon_{0}(\delta)$ and for all $h>0$ such that

$$
\left\|U_{\varepsilon}^{0}\right\|_{0, \infty} \leq 1-\frac{1}{2} \delta \quad \text { and } \quad\left|u_{0}-U_{\varepsilon}^{0}\right|_{1}^{2} \leq C \Delta t,
$$

the unique solution $\left\{U_{\varepsilon}^{n}, W_{\varepsilon}^{n}\right\}_{n=0}^{N}$ to $\left(\mathrm{P}_{\varepsilon}^{h, \Delta t}\right)$ satisfies

$$
\begin{aligned}
\Delta t \sum_{n=1}^{N}\left\|\frac{U_{\varepsilon}^{n}-U_{\varepsilon}^{n-1}}{\Delta t}\right\|_{1}^{2} & +(\Delta t)^{2} \sum_{n=1}^{N}\left|\frac{W_{\varepsilon}^{n}-W_{\varepsilon}^{n-1}}{\Delta t}\right|_{1}^{2} \\
& +\max _{n=1 \rightarrow N}\left|W_{\varepsilon}^{n}\right|_{1}^{2}+\max _{n=1 \rightarrow N}\left|\hat{\mathcal{G}}^{h}\left(\frac{U_{\varepsilon}^{n}-U_{\varepsilon}^{n-1}}{\Delta t}\right)\right|_{1}^{2} \leq C_{b} .
\end{aligned}
$$

Proof. The proof is a discrete analogue of Corollary 2.1. From (3.1b),(3.1c) it follows for $n \geq 1$ that

$$
\begin{aligned}
\left(W_{\varepsilon}^{n}-W_{\varepsilon}^{n-1}, \chi\right)^{h}= & \gamma\left(\nabla\left(U_{\varepsilon}^{n}-U_{\varepsilon}^{n-1}\right), \nabla \chi\right)+\left(\phi_{\varepsilon}\left(U_{\varepsilon}^{n}\right)-\phi_{\varepsilon}\left(U_{\varepsilon}^{n-1}\right), \chi\right)^{h} \\
& -\Delta t \theta_{c}\left(Y_{\varepsilon}^{n}, \chi\right)^{h}-\gamma\left(\nabla Z_{\varepsilon}^{n}, \nabla \chi\right) \quad \forall \chi \in S^{h},
\end{aligned}
$$

where

$$
Y_{\varepsilon}^{n}:=\left(U_{\varepsilon}^{n}-U_{\varepsilon}^{n-1}\right) / \Delta t \quad n \geq 1 \quad \text { and } \quad Z_{\varepsilon}^{n}:= \begin{cases}u_{0}-U_{\varepsilon}^{0} & n=1 \\ 0 & n \geq 2 .\end{cases}
$$

Choosing $\chi \equiv U_{\varepsilon}^{n}-U_{\varepsilon}^{n-1}$ in (3.43) and noting (3.1a) with $\chi \equiv W_{\varepsilon}^{n}-W_{\varepsilon}^{n-1}$ yields for $n \geq 1$ that

$$
\begin{aligned}
\gamma \Delta t\left|Y_{\varepsilon}^{n}\right|_{1}^{2} & +\left(\phi_{\varepsilon}\left(U_{\varepsilon}^{n}\right)-\phi_{\varepsilon}\left(U_{\varepsilon}^{n-1}\right), Y_{\varepsilon}^{n}\right)^{h} \\
& +b_{\max }\left|W_{\varepsilon}^{n}-W_{\varepsilon}^{n-1}\right|_{1}^{2} \\
= & -\left(b\left(U_{\varepsilon}^{n-1}\right) \nabla W_{\varepsilon}^{n-1}, \nabla\left(W_{\varepsilon}^{n}-W_{\varepsilon}^{n-1}\right)\right) \\
& +\theta_{c} \Delta t\left|Y_{\varepsilon}^{n}\right|_{h}^{2}+\gamma\left(\nabla Z_{\varepsilon}^{n}, \nabla Y_{\varepsilon}^{n}\right) .
\end{aligned}
$$

It follows from (3.45) on noting (2.6), (2.5) and (3.19) that for $n \geq 1$

$$
\begin{aligned}
\gamma \Delta t \mid & \left.Y_{\varepsilon}^{n}\right|_{1} ^{2}+\theta \Delta t\left|Y_{\varepsilon}^{n}\right|_{h}^{2}+\frac{1}{2} b_{\max }(\Delta t)^{2}\left|\frac{W_{\varepsilon}^{n}-W_{\varepsilon}^{n-1}}{\Delta t}\right|_{1}^{2}+\frac{1}{2}\left|\left[b\left(U_{\varepsilon}^{n-1}\right)\right]^{\frac{1}{2}} \nabla W_{\varepsilon}^{n}\right|_{0}^{2} \\
\leq & \gamma \Delta t\left|Y_{\varepsilon}^{n}\right|_{1}^{2}+\Delta t\left(\frac{\phi_{\varepsilon}\left(U_{\varepsilon}^{n}\right)-\phi_{\varepsilon}\left(U_{\varepsilon}^{n-1}\right)}{\Delta t}, Y_{\varepsilon}^{n}\right)^{h} \\
& +(\Delta t)^{2}\left|\left[b_{\max }-\frac{1}{2} b\left(U_{\varepsilon}^{n-1}\right)\right]^{\frac{1}{2}} \nabla\left(\frac{W_{\varepsilon}^{n}-W_{\varepsilon}^{n-1}}{\Delta t}\right)\right|_{0}^{2}+\frac{1}{2}\left|\left[b\left(U_{\varepsilon}^{n-1}\right)\right]^{\frac{1}{2}} \nabla W_{\varepsilon}^{n}\right|_{0}^{2} \\
= & \theta_{c} \Delta t\left|Y_{\varepsilon}^{n}\right|_{h}^{2}+\frac{1}{2}\left|\left[b\left(U_{\varepsilon}^{n-1}\right)\right]^{\frac{1}{2}} \nabla W_{\varepsilon}^{n-1}\right|_{0}^{2}+\gamma\left(\nabla Z_{\varepsilon}^{n}, \nabla Y_{\varepsilon}^{n}\right) \\
(3.46) \leq & \frac{\gamma}{2} \Delta t\left|Y_{\varepsilon}^{n}\right|_{1}^{2}+C \Delta t\left|\hat{\mathcal{G}}^{h} Y_{\varepsilon}^{n}\right|_{1}^{2}+p_{n},
\end{aligned}
$$

where $p_{1}:=\frac{1}{2}\left|\left[b\left(U_{\varepsilon}^{0}\right)\right]^{\frac{1}{2}} \nabla W_{\varepsilon}^{0}\right|_{0}^{2}+C \frac{1}{\Delta t}\left|Z_{\varepsilon}^{1}\right|_{1}^{2}$ and

$$
p_{n}:=\frac{1}{2}\left|\left[b\left(U_{\varepsilon}^{n-2}\right)\right]^{\frac{1}{2}} \nabla W_{\varepsilon}^{n-1}\right|_{0}^{2}+\frac{1}{2} \Delta t \int_{\Omega}\left[\frac{b\left(U_{\varepsilon}^{n-1}\right)-b\left(U_{\varepsilon}^{n-2}\right)}{\Delta t}\right]\left|\nabla W_{\varepsilon}^{n-1}\right|^{2} \mathrm{~d} x \text { if } n \geq 2 .
$$

Similarly to (2.48) and alternatively to (2.49), we have from (1.4b), (2.15), (2.16), (3.11) and a Young's inequality that for $n \geq 2$

$$
\begin{aligned}
& \int_{\Omega}\left[\frac{b\left(U_{\varepsilon}^{n-1}\right)-b\left(U_{\varepsilon}^{n-2}\right)}{\Delta t}\right]\left|\nabla W_{\varepsilon}^{n-1}\right|^{2} \mathrm{~d} x \leq \frac{\gamma}{2}\left|Y_{\varepsilon}^{n-1}\right|_{1}^{2}+C\left|W_{\varepsilon}^{n-1}\right|_{1,2(1+\tau)}^{4} \\
& \quad \leq \frac{\gamma}{2}\left|Y_{\varepsilon}^{n-1}\right|_{1}^{2}+C h^{-\frac{2 \tau d}{1+\tau}}\left|W_{\varepsilon}^{n-1}\right|_{1}^{4} \\
& \quad \leq \frac{\gamma}{2}\left|Y_{\varepsilon}^{n-1}\right|_{1}^{2}+C\left|W_{\varepsilon}^{n-1}\right|_{1}^{4(1+\tau)}+C \tau h^{-2 d}
\end{aligned}
$$


where $\tau=0$ for $d=1$, and for any $\tau>0$ for $d=2$. We set $s_{0}:=0, v_{0}:=1$ and

$$
r_{n}:=\frac{\gamma}{2} \Delta t\left|Y_{\varepsilon}^{n}\right|_{1}^{2}, \quad s_{n}:=\left|\left[b\left(U_{\varepsilon}^{n-1}\right)\right]^{\frac{1}{2}} \nabla W_{\varepsilon}^{n}\right|_{0}^{2}, \quad v_{n}:=\max \left\{r_{n}+s_{n}, v_{n-1}\right\}
$$

$$
y_{n}:=C\left|\hat{\mathcal{G}}^{h} Y_{\varepsilon}^{n}\right|_{1}^{2}+C \tau h^{-2 d}, \quad z_{n}:=C s_{n-1}+y_{n} \quad n \geq 1 .
$$

It follows from (3.28) and if $\tau \leq C h^{2 d}$ that

$$
\Delta t \sum_{n=1}^{N} z_{n} \leq C \Delta t \sum_{n=1}^{N} s_{n}+\Delta t \sum_{n=1}^{N} y_{n} \leq C_{b}\left(1+\tau h^{-2 d}\right) \leq C_{b} .
$$

It follows from (3.48), (3.46), (3.47), (3.28) and (3.41) that $v_{1} \leq C_{b}$ and

(3.50) $\max \left\{1, s_{n-1}\right\} \leq v_{n-1} \leq v_{n} \leq v_{n-1}+C_{b} \Delta t s_{n-1}^{2(1+\tau)}+\Delta t y_{n} \quad n \geq 2$.

For $d=1$, i.e., $\tau=0$, it follows from (3.50) and (3.49) that

$$
\begin{aligned}
v_{n} & \leq\left(1+C_{b} \Delta t s_{n-1}\right) v_{n-1}+\Delta t y_{n} \leq e^{C_{b} \Delta t s_{n-1}} v_{n-1}+\Delta t y_{n} \\
& \leq e^{C_{b} \Delta t \sum_{i=1}^{n-1} s_{i}}\left[v_{1}+\Delta t \sum_{i=2}^{n} y_{i}\right] \leq C_{b} \quad n=2 \rightarrow N .
\end{aligned}
$$

For $d=2$, i.e., $\tau>0$, it follows from the mean value theorem, (3.50) and (3.48) that

$$
-\frac{1}{2 \tau}\left[v_{n}^{-2 \tau}-v_{n-1}^{-2 \tau}\right] \leq v_{n-1}^{-(1+2 \tau)}\left[v_{n}-v_{n-1}\right] \leq \Delta t z_{n} \quad n \geq 2 .
$$

Summing (3.52) and noting (3.49) yields that $v_{1} \leq C_{b}$ and

$$
\begin{aligned}
v_{n} & \leq\left[1-2 \tau v_{1}^{2 \tau} \Delta t \sum_{i=2}^{n} z_{i}\right]^{-\frac{1}{2 \tau}} v_{1} \\
& \leq\left[1+4 \tau v_{1}^{2 \tau} \Delta t \sum_{i=2}^{n} z_{i}\right]^{\frac{1}{2 \tau}} v_{1} \leq e^{2 v_{1}^{2 \tau} \Delta t \sum_{i=2}^{N} z_{i}} v_{1} \leq C_{b} \quad n=2 \rightarrow N ;
\end{aligned}
$$

provided $\tau>0$ is chosen sufficiently small so that $\tau \leq C h^{2 d}$ and

$$
4 \tau v_{1}^{2 \tau} \Delta t \sum_{n=1}^{N} z_{n} \leq 1 .
$$

Hence the third bound in (3.42) follows from (3.48), (3.51) and (3.53). The fourth bound in (3.42) follows from the third on noting (3.25) and (3.26). The first two bounds in (3.42) follow from summing (3.46) and noting (3.47), the third bound in (3.42), (3.28), (3.41) and (2.13).

Assuming that $b_{\min }>0$ and given $q$ measurable in $\Omega$, we introduce the analogue of (2.17): $\mathcal{G}_{q}^{h}: \mathcal{F} \rightarrow V^{h}$ such that

$$
\left(b(q) \nabla \mathcal{G}_{q}^{h} v, \nabla \chi\right)=\langle v, \chi\rangle \quad \forall \chi \in S^{h} .
$$

The analogues of (2.18) and (2.19) hold: for all $v \in \mathcal{F}$

$$
b_{\min }\left|[b(q)]^{\frac{1}{2}} \nabla \mathcal{G}_{q}^{h} v\right|_{0}^{2} \leq\left|\nabla \mathcal{G}^{h} v\right|_{0}^{2} \leq b_{\max }\left|[b(q)]^{\frac{1}{2}} \nabla \mathcal{G}_{q}^{h} v\right|_{0}^{2},
$$

and

$$
\langle v, \chi\rangle \equiv\left(b(q) \nabla \mathcal{G}_{q}^{h} v, \nabla \chi\right) \leq b_{\max }^{\frac{1}{2}}\left\langle v, \mathcal{G}_{q}^{h} v\right\rangle^{\frac{1}{2}}|\chi|_{1} \quad \forall \chi \in S^{h}
$$


It follows immediately from (2.17), (3.55) and (3.5) that for all measurable $q$ and $v \in \mathcal{F}$ that

$$
\left|[b(q)]^{\frac{1}{2}} \nabla\left(\mathcal{G}_{q}-\mathcal{G}_{q}^{h}\right) v\right|_{0} \leq\left|[b(q)]^{\frac{1}{2}} \nabla\left(I-\pi^{h}\right) \mathcal{G}_{q} v\right|_{0} \leq C h\left|\mathcal{G}_{q} v\right|_{2} .
$$

Similarly to (3.55), we introduce $\hat{\mathcal{G}}_{q}^{h}: \mathcal{F}^{h} \rightarrow V^{h}$ such that

$$
\left(b(q) \nabla \hat{\mathcal{G}}_{q}^{h} v, \nabla \chi\right)=(v, \chi)^{h} \quad \forall \chi \in S^{h} .
$$

The analogues of (3.56) and (3.57) hold: for all $v \in \mathcal{F}^{h}$

$$
b_{\min }\left|[b(q)]^{\frac{1}{2}} \nabla \hat{\mathcal{G}}_{q}^{h} v\right|_{0}^{2} \leq\left|\nabla \hat{\mathcal{G}}^{h} v\right|_{0}^{2} \leq b_{\max }\left|[b(q)]^{\frac{1}{2}} \nabla \hat{\mathcal{G}}_{q}^{h} v\right|_{0}^{2},
$$

and

$$
(v, \chi)^{h} \equiv\left(b(q) \nabla \hat{\mathcal{G}}_{q}^{h} v, \nabla \chi\right) \leq b_{\max }^{\frac{1}{2}}\left[\left(v, \hat{\mathcal{G}}_{q}^{h} v\right)^{h}\right]^{\frac{1}{2}}|\chi|_{1} \quad \forall \chi \in S^{h} .
$$

An analogue of (2.24) holds: for $q_{1}, q_{2} \in L^{4}(\Omega)$ and $v \in \mathcal{F}^{h}$

$$
\left|\nabla\left(\hat{\mathcal{G}}_{q_{1}}^{h}-\hat{\mathcal{G}}_{q_{2}}^{h}\right) v\right|_{0} \leq C b_{\min }^{-1}\left|q_{1}-q_{2}\right|_{0,4}\left|\nabla \hat{\mathcal{G}}_{q_{2}}^{h} v\right|_{0,4} .
$$

Similarly to (3.20), it is easily deduced from (3.8) that for $q$ measurable

$$
\left\|\left(\mathcal{G}_{q}^{h}-\hat{\mathcal{G}}_{q}^{h}\right) v^{h}\right\|_{1} \leq C b_{\min }^{-1} h^{2}\left\|v^{h}\right\|_{1} \quad \forall v^{h} \in V^{h} .
$$

We now prove an error estimate between the problems $\left(\mathrm{P}_{\varepsilon}^{h, \Delta t}\right)$ and $\left(\mathrm{P}_{\varepsilon}\right)$.

Theorem 3.1. Let the assumptions of Lemma 3.2 hold. Then we have that

$$
\left\|u_{\varepsilon}-U_{\varepsilon}^{+}\right\|_{L^{2}\left(0, T ; H^{1}(\Omega)\right)}^{2}+\left\|u_{\varepsilon}-U_{\varepsilon}\right\|_{L^{\infty}\left(0, T ;\left(H^{1}(\Omega)\right)^{\prime}\right)}^{2} \leq C_{b}\left[\varepsilon^{-1} h^{2}+\Delta t\right],
$$

where

$$
U_{\varepsilon}(\cdot, t):=\frac{t-t_{n-1}}{\Delta t} U_{\varepsilon}^{n}(\cdot)+\frac{t_{n}-t}{\Delta t} U_{\varepsilon}^{n-1}(\cdot) \quad t \in\left[t_{n-1}, t_{n}\right] n \geq 1
$$

and

$$
U_{\varepsilon}^{+}(\cdot, t):=U_{\varepsilon}^{n}(\cdot), \quad U_{\varepsilon}^{-}(\cdot, t):=U_{\varepsilon}^{n-1}(\cdot) \quad t \in\left(t_{n-1}, t_{n}\right] n \geq 1 .
$$

Proof. Using the above notation and introducing analogous notation for $W_{\varepsilon},(3.1 \mathrm{a})-$ (3.1c) can be restated as follows.

Find $\left\{U_{\varepsilon}, W_{\varepsilon}\right\} \in H^{1}\left(0, T ; S^{h}\right) \times L^{\infty}\left(0, T ; S^{h}\right)$ such that $U_{\varepsilon}(\cdot, 0) \equiv Q^{h} u_{0}(\cdot)$ or $\hat{Q}^{h} u_{0}(\cdot), W_{\varepsilon}(\cdot, 0)$ is defined by $(3.1 \mathrm{c})$ and for a.e. $t \in(0, T),\left(U_{\varepsilon}(\cdot, t), 1\right)=\left(u_{0}(\cdot), 1\right)$ and

$$
\begin{array}{r}
\gamma\left(\nabla U_{\varepsilon}^{+}, \nabla \chi\right)+\left(\phi_{\varepsilon}\left(U_{\varepsilon}^{+}\right)-\theta_{c} U_{\varepsilon}^{+},(I-f) \chi\right)^{h}+\left(\hat{\mathcal{G}}_{U_{\varepsilon}^{-}}^{h} \frac{\partial U_{\varepsilon}}{\partial t}, \chi\right)^{h} \\
=\Delta t\left(\left[b\left(U_{\varepsilon}^{-}\right)-b_{\max }\right] \nabla \frac{\partial W_{\varepsilon}}{\partial t}, \nabla \hat{\mathcal{G}}_{U_{\varepsilon}^{-}}^{h}(I-f) \chi\right) \quad \forall \chi \in S^{h}, \\
\left(W_{\varepsilon}^{+}, \chi\right)^{h}=\gamma\left(\nabla U_{\varepsilon}^{+}, \nabla \chi\right)+\left(\phi_{\varepsilon}\left(U_{\varepsilon}^{+}\right)-\theta_{c} U_{\varepsilon}^{+}, \chi\right)^{h} \quad \forall \chi \in S^{h} .
\end{array}
$$

Note that (3.65a) follows by combining (3.1b) with $\chi \equiv(I-f) \chi$ and (3.1a) with $\chi \equiv \hat{\mathcal{G}}_{U_{\varepsilon}^{-}}^{h}(I-f) \chi$. We set $e_{\varepsilon}^{( \pm)}:=u_{\varepsilon}-U_{\varepsilon}^{( \pm)} \in V, e_{\varepsilon}^{A}:=u_{\varepsilon}-\pi^{h} u_{\varepsilon}$ and $E_{\varepsilon}^{( \pm)}:=\pi^{h} u_{\varepsilon}-U_{\varepsilon}^{( \pm)} \in S^{h}$ for a.e. $t \in(0, T)$. We note for future reference that

$$
U_{\varepsilon}-U_{\varepsilon}^{ \pm}=\left(t-\hat{t}_{n}^{ \pm}\right) \frac{\partial U_{\varepsilon}}{\partial t} \quad t \in\left(t_{n-1}, t_{n}\right) n \geq 1,
$$

where $\hat{t}_{n}^{+}:=t_{n}$ and $\hat{t}_{n}^{-}:=t_{n-1}$. 
On subtracting (3.65a) from (2.28), it follows for a.e. $t \in(0, T)$ that

$$
\begin{aligned}
\gamma\left(\nabla e_{\varepsilon}^{+}\right. & , \nabla \chi)+\left(\phi_{\varepsilon}\left(u_{\varepsilon}\right)-\phi_{\varepsilon}\left(U_{\varepsilon}^{+}\right),(I-f) \chi\right)+\left(\mathcal{G}_{u_{\varepsilon}} \frac{\partial e_{\varepsilon}}{\partial t}, \chi\right) \\
= & \theta_{c}\left(e_{\varepsilon}^{+},(I-f) \chi\right) \\
& +\left[\left(\phi_{\varepsilon}\left(U_{\varepsilon}^{+}\right)-\theta_{c} U_{\varepsilon}^{+},(I-f) \chi\right)^{h}-\left(\phi_{\varepsilon}\left(U_{\varepsilon}^{+}\right)-\theta_{c} U_{\varepsilon}^{+},(I-f) \chi\right)\right] \\
& +\left[\left(\hat{\mathcal{G}}_{U_{\varepsilon}^{-}}^{h} \frac{\partial U_{\varepsilon}}{\partial t}, \chi\right)^{h}-\left(\mathcal{G}_{u_{\varepsilon}} \frac{\partial U_{\varepsilon}}{\partial t}, \chi\right)\right] \\
& +\Delta t\left(\left[b_{\max }-b\left(U_{\varepsilon}^{-}\right)\right] \nabla \frac{\partial W_{\varepsilon}}{\partial t}, \nabla \hat{\mathcal{G}}_{U_{\varepsilon}^{-}}^{h}(I-f) \chi\right) \quad \forall \chi \in S^{h} .
\end{aligned}
$$

Hence choosing $\chi \equiv E_{\varepsilon}^{+} \in S^{h}$ and noting (2.7), (2.15), (2.13), (2.18), (3.8), (3.16) and a Young's inequality, yields for a.e. $t \in(0, T)$ that

$$
\begin{aligned}
\gamma\left|e_{\varepsilon}^{+}\right|_{1}^{2}+ & \frac{\varepsilon}{\theta}\left|\phi_{\varepsilon}\left(u_{\varepsilon}\right)-\phi_{\varepsilon}\left(U_{\varepsilon}^{+}\right)\right|_{0}^{2}+\left(\mathcal{G}_{u_{\varepsilon}} \frac{\partial e_{\varepsilon}}{\partial t}, e_{\varepsilon}^{+}\right) \\
\leq & \gamma\left|e_{\varepsilon}^{+}\right|_{1}^{2}+\left(\phi_{\varepsilon}\left(u_{\varepsilon}\right)-\phi_{\varepsilon}\left(U_{\varepsilon}^{+}\right), e_{\varepsilon}^{+}\right)+\left(\mathcal{G}_{u_{\varepsilon}} \frac{\partial e_{\varepsilon}}{\partial t}, e_{\varepsilon}^{+}\right) \\
= & \theta_{c}\left(e_{\varepsilon}^{+}, \bar{E}_{\varepsilon}^{+}\right)+\gamma\left(\nabla e_{\varepsilon}^{+}, \nabla e_{\varepsilon}^{A}\right)+\left(\phi_{\varepsilon}\left(u_{\varepsilon}\right)-\phi_{\varepsilon}\left(U_{\varepsilon}^{+}\right),(I-f) e_{\varepsilon}^{A}\right)+\left(\mathcal{G}_{u_{\varepsilon}} \frac{\partial e_{\varepsilon}}{\partial t}, e_{\varepsilon}^{A}\right) \\
& +\left[\left(\pi^{h}\left[\phi_{\varepsilon}\left(U_{\varepsilon}^{+}\right)\right]-\theta_{c} U_{\varepsilon}^{+}, \bar{E}_{\varepsilon}^{+}\right)^{h}-\left(\pi^{h}\left[\phi_{\varepsilon}\left(U_{\varepsilon}^{+}\right)\right]-\theta_{c} U_{\varepsilon}^{+}, \bar{E}_{\varepsilon}^{+}\right)\right] \\
& +\left(\left(\pi^{h}-I\right)\left[\phi_{\varepsilon}\left(U_{\varepsilon}^{+}\right)\right], \bar{E}_{\varepsilon}^{+}\right)+\left[\left(\hat{\mathcal{G}}_{U_{\varepsilon}^{-}}^{h} \frac{\partial U_{\varepsilon}}{\partial t}, E_{\varepsilon}^{+}\right)^{h}-\left(\mathcal{G}_{u_{\varepsilon}} \frac{\partial U_{\varepsilon}}{\partial t}, E_{\varepsilon}^{+}\right)\right] \\
& +\Delta t\left(\left[b_{\max }-b\left(U_{\varepsilon}^{-}\right)\right] \nabla \frac{\partial W_{\varepsilon}}{\partial t}, \nabla \hat{\mathcal{G}}_{U_{\varepsilon}}^{h} \bar{E}_{\varepsilon}^{+}\right) \\
\leq & C_{b}\left[\left\|e_{\varepsilon}^{+}\right\|_{-1}^{2}+\left|e_{\varepsilon}^{A}\right|_{1}^{2}+\varepsilon^{-1}\left|e_{\varepsilon}^{A}\right|_{0}^{2}+\left\|\frac{\partial e_{\varepsilon}}{\partial t}\right\|{ }_{-1}\left|e_{\varepsilon}^{A}\right|_{0}\right. \\
& \left.+h^{4}\left\|U_{\varepsilon}^{+}\right\|_{1}^{2}+h^{2}\left\|\pi^{h}\left[\phi_{\varepsilon}\left(U_{\varepsilon}^{+}\right)\right]\right\|_{1}^{2}\right]+\left[\left(\frac{\partial U_{\varepsilon}}{\partial t}, \hat{\mathcal{G}}_{U_{\varepsilon}}^{h} \bar{E}_{\varepsilon}^{+}\right)^{h}-\left(\frac{\partial U_{\varepsilon}}{\partial t}, \hat{\mathcal{G}}_{U_{\varepsilon}^{-}}^{h} \bar{E}_{\varepsilon}^{+}\right)\right] \\
& +\left(\frac{\partial U_{\varepsilon}}{\partial t},\left(\hat{\mathcal{G}}_{U_{\varepsilon}^{-}}^{h}-\mathcal{G}_{u_{\varepsilon}}\right) \bar{E}_{\varepsilon}^{+}\right)+\Delta t\left(\left[b_{\max }-b\left(U_{\varepsilon}^{-}\right)\right] \nabla \frac{\partial W_{\varepsilon}}{\partial t}, \nabla \hat{\mathcal{G}}_{U_{\varepsilon}}^{h} \bar{E}_{\varepsilon}^{+}\right),
\end{aligned}
$$

where $\bar{E}_{\varepsilon}^{+}:=(I-f) E_{\varepsilon}^{+}$.

From (3.8), (3.60), (3.21), (3.10) and (2.16) it follows that

$$
\begin{aligned}
& \left|\left(\frac{\partial U_{\varepsilon}}{\partial t}, \hat{\mathcal{G}}_{U_{\varepsilon}^{-}}^{h} \bar{E}_{\varepsilon}^{+}\right)^{h}-\left(\frac{\partial U_{\varepsilon}}{\partial t}, \hat{\mathcal{G}}_{U_{\varepsilon}^{-}}^{h} \bar{E}_{\varepsilon}^{+}\right)\right| \\
& \quad \leq C h^{2}\left\|\frac{\partial U_{\varepsilon}}{\partial t}\right\|_{1}\left|\hat{\mathcal{G}}_{U_{\varepsilon}^{-}}^{h} \bar{E}_{\varepsilon}^{+}\right|_{1} \leq C_{b}\left[\left\|e_{\varepsilon}^{+}\right\|_{-1}^{2}+\left|e_{\varepsilon}^{A}\right|_{0}^{2}\right]+C h^{4}\left|\frac{\partial U_{\varepsilon}}{\partial t}\right|_{1}^{2} .
\end{aligned}
$$

Similarly from (3.60), (3.21), (3.10) and (2.16) it follows that

$$
\Delta t\left|\left(\left[b_{\max }-b\left(U_{\varepsilon}^{-}\right)\right] \nabla \frac{\partial W_{\varepsilon}}{\partial t}, \nabla \hat{\mathcal{G}}_{U_{\varepsilon}^{-}}^{h} \bar{E}_{\varepsilon}^{+}\right)\right| \leq C_{b}\left[\left\|e_{\varepsilon}^{+}\right\|_{-1}^{2}+\left|e_{\varepsilon}^{A}\right|_{0}^{2}\right]+C(\Delta t)^{2}\left|\frac{\partial W_{\varepsilon}}{\partial t}\right|_{1}^{2} .
$$

From (2.15) it follows that

$$
\begin{aligned}
& \left|\left(\frac{\partial U_{\varepsilon}}{\partial t},\left(\hat{\mathcal{G}}_{U_{\varepsilon}^{-}}^{h}-\mathcal{G}_{u_{\varepsilon}}\right) \bar{E}_{\varepsilon}^{+}\right)\right| \\
& \quad \leq C\left\|\frac{\partial U_{\varepsilon}}{\partial t}\right\|_{-1}\left[\left|\left(\mathcal{G}_{u_{\varepsilon}}-\hat{\mathcal{G}}_{u_{\varepsilon}}^{h}\right) \bar{E}_{\varepsilon}^{+}\right|_{1}+\left|\left(\hat{\mathcal{G}}_{u_{\varepsilon}}^{h}-\hat{\mathcal{G}}_{U_{\varepsilon}^{-}}^{h}\right) \bar{E}_{\varepsilon}^{+}\right|_{1}\right] .
\end{aligned}
$$

From $(2.26),(2.46 \mathrm{~b})$ and $(2.20)$ it follows that

$$
\left\|\mathcal{G}_{u_{\varepsilon}} v\right\|_{2} \leq C_{b}|v|_{0} \quad \forall v \in L^{2}(\Omega) \cap \mathcal{F} .
$$


From (3.58), (3.72), (3.63), (3.10) and (2.15) it follows that

$$
\begin{aligned}
\left|\left(\mathcal{G}_{u_{\varepsilon}}-\hat{\mathcal{G}}_{u_{\varepsilon}}^{h}\right) \bar{E}_{\varepsilon}^{+}\right|_{1} & \leq\left|\left(\mathcal{G}_{u_{\varepsilon}}-\mathcal{G}_{u_{\varepsilon}}^{h}\right) \bar{E}_{\varepsilon}^{+}\right|_{1}+\left|\left(\mathcal{G}_{u_{\varepsilon}}^{h}-\hat{\mathcal{G}}_{u_{\varepsilon}}^{h}\right) \bar{E}_{\varepsilon}^{+}\right|_{1} \\
& \leq C_{b}\left[h\left|\bar{E}_{\varepsilon}^{+}\right|_{0}+h^{2}\left\|\bar{E}_{\varepsilon}^{+}\right\|_{1}\right] \leq C_{b} h\left|\bar{E}_{\varepsilon}^{+}\right|_{0} \\
& \leq C_{b}\left[\left\|e_{\varepsilon}^{+}\right\|_{-1}\left|e_{\varepsilon}^{+}\right|_{1}+\left|e_{\varepsilon}^{A}\right|_{0}^{2}+h^{2}\right] .
\end{aligned}
$$

From (3.62) it follows that

$$
\left|\left(\hat{\mathcal{G}}_{u_{\varepsilon}}^{h}-\hat{\mathcal{G}}_{U_{\varepsilon}^{-}}^{h}\right) \bar{E}_{\varepsilon}^{+}\right|_{1} \leq C b_{\text {min }}^{-1}\left|e_{\varepsilon}^{-}\right|_{0,4}\left|\hat{\mathcal{G}}_{u_{\varepsilon}}^{h} \bar{E}_{\varepsilon}^{+}\right|_{1,4} .
$$

From (3.11) and (3.73) it follows that

$$
\begin{aligned}
\left|\hat{\mathcal{G}}_{u_{\varepsilon}}^{h} \bar{E}_{\varepsilon}^{+}\right|_{1,4} & \leq\left|\mathcal{G}_{u_{\varepsilon}} \bar{E}_{\varepsilon}^{+}\right|_{1,4}+\left|\left(\mathcal{G}_{u_{\varepsilon}}-\mathcal{G}_{u_{\varepsilon}}^{h}\right) \bar{E}_{\varepsilon}^{+}\right|_{1,4}+C h^{-\frac{d}{4}}\left|\left(\mathcal{G}_{u_{\varepsilon}}^{h}-\hat{\mathcal{G}}_{u_{\varepsilon}}^{h}\right) \bar{E}_{\varepsilon}^{+}\right|_{1} \\
& \leq\left|\mathcal{G}_{u_{\varepsilon}} \bar{E}_{\varepsilon}^{+}\right|_{1,4}+\left|\left(\mathcal{G}_{u_{\varepsilon}}-\mathcal{G}_{u_{\varepsilon}}^{h}\right) \bar{E}_{\varepsilon}^{+}\right|_{1,4}+C_{b} h^{1-\frac{d}{4}}\left|\bar{E}_{\varepsilon}^{+}\right|_{0} .
\end{aligned}
$$

From (3.11), (3.5), (3.72) and (3.73) it follows that

$$
\begin{aligned}
\mid\left(\mathcal{G}_{u_{\varepsilon}}\right. & \left.-\mathcal{G}_{u_{\varepsilon}}^{h}\right)\left.\bar{E}_{\varepsilon}^{+}\right|_{1,4} \leq\left|\left(I-\pi^{h}\right) \mathcal{G}_{u_{\varepsilon}} \bar{E}_{\varepsilon}^{+}\right|_{1,4}+C h^{-\frac{d}{4}}\left|\left(\pi^{h} \mathcal{G}_{u_{\varepsilon}}-\mathcal{G}_{u_{\varepsilon}}^{h}\right) \bar{E}_{\varepsilon}^{+}\right|_{1} \\
& \leq C_{b} h^{-\frac{d}{4}}\left[h\left|\bar{E}_{\varepsilon}^{+}\right|_{0}+\left|\left(I-\pi^{h}\right) \mathcal{G}_{u_{\varepsilon}} \bar{E}_{\varepsilon}^{+}\right|_{1}+\left|\left(\mathcal{G}_{u_{\varepsilon}}-\mathcal{G}_{u_{\varepsilon}}^{h}\right) \bar{E}_{\varepsilon}^{+}\right|_{1}\right] \\
& \leq C_{b} h^{1-\frac{d}{4}}\left|\bar{E}_{\varepsilon}^{+}\right|_{0} .
\end{aligned}
$$

Combining (3.75) and (3.76), and noting (2.10), (3.72), (2.18), (3.10) and (2.15) yields that

$$
\begin{aligned}
\left|\hat{\mathcal{G}}_{u_{\varepsilon}}^{h} \bar{E}_{\varepsilon}^{+}\right|_{1,4} & \leq\left|\mathcal{G}_{u_{\varepsilon}} \bar{E}_{\varepsilon}^{+}\right|_{1,4}+C_{b} h^{1-\frac{d}{4}}\left|\bar{E}_{\varepsilon}^{+}\right|_{0} \leq C_{b}\left\|\bar{E}_{\varepsilon}^{+}\right\|_{-1}^{1-\frac{d}{4}}\left|\bar{E}_{\varepsilon}^{+}\right|_{0}^{\frac{d}{4}} \\
& \leq C_{b}\left\|\bar{E}_{\varepsilon}^{+}\right\|_{-1}^{1-\frac{d}{4}}\left[\left|e_{\varepsilon}^{A}\right|_{0}^{\frac{d}{4}}+\left\|e_{\varepsilon}^{+}\right\|_{-1}^{\frac{d}{8}}\left|e_{\varepsilon}^{+}\right|_{1}^{\frac{d}{8}}\right] .
\end{aligned}
$$

From (3.66), (2.10) and (2.15) it follows that

$$
\left|e_{\varepsilon}^{-}\right|_{0,4} \leq\left|e_{\varepsilon}^{+}\right|_{0,4}+\Delta t\left|\frac{\partial U_{\varepsilon}}{\partial t}\right|_{0,4} \leq C\left[\left\|e_{\varepsilon}^{+}\right\|_{-1}^{\frac{4-d}{8}}\left|e_{\varepsilon}^{+}\right|_{1}^{\frac{4+d}{8}}+\Delta t\left|\frac{\partial U_{\varepsilon}}{\partial t}\right|_{1}\right] .
$$

Combining (3.71), (3.73), (3.74), (3.77), (3.78) and noting (3.66) yields that

$$
\left|\left(\frac{\partial U_{\varepsilon}}{\partial t},\left(\hat{\mathcal{G}}_{U_{\varepsilon}^{-}}^{h}-\mathcal{G}_{u_{\varepsilon}}\right) \bar{E}_{\varepsilon}^{+}\right)\right|
$$

$(3.79) \leq \frac{1}{4} \gamma\left|e_{\varepsilon}^{+}\right|_{1}^{2}+C_{b}\left[1+\left\|\frac{\partial U_{\varepsilon}}{\partial t}\right\|_{-1}^{2}\right]\left[\left\|e_{\varepsilon}\right\|_{-1}^{2}+\left|e_{\varepsilon}^{A}\right|_{0}^{2}+(\Delta t)^{2}\left|\frac{\partial U_{\varepsilon}}{\partial t}\right|_{1}^{2}+h^{2}\right]$.

We note from (2.23), (2.18), (3.72), (2.15) and (3.66) that

$$
\begin{gathered}
\left|\left(b^{\prime}\left(u_{\varepsilon}\right) \frac{\partial u_{\varepsilon}}{\partial t} \nabla \mathcal{G}_{u_{\varepsilon}} e_{\varepsilon}, \nabla \mathcal{G}_{u_{\varepsilon}} e_{\varepsilon}\right)\right| \leq C_{b}\left|\frac{\partial u_{\varepsilon}}{\partial t}\right|_{0}\left\|e_{\varepsilon}\right\|_{-1}^{2-\frac{d}{4}}\left|e_{\varepsilon}\right|_{1}^{\frac{d}{4}} \\
(3.80) \leq \frac{1}{4} \gamma\left|e_{\varepsilon}^{+}\right|_{1}^{2}+C_{b}\left[\left|\frac{\partial u_{\varepsilon}}{\partial t}\right|_{0}^{\frac{8}{8-d}} \|\left. e_{\varepsilon}\right|_{-1} ^{2}+(\Delta t)^{2}\left|\frac{\partial U_{\varepsilon}}{\partial t}\right|_{1}^{2}\right] .
\end{gathered}
$$

Combining (3.68), (3.69), (3.70), (3.79) and noting (2.22), (3.66), (3.80) and (2.18) yields that

$$
\begin{aligned}
& \gamma\left|e_{\varepsilon}^{+}\right|_{1}^{2}+\frac{1}{2} \frac{\mathrm{d}}{\mathrm{d} t}\left(\mathcal{G}_{u_{\varepsilon}} e_{\varepsilon}, e_{\varepsilon}\right)=\gamma\left|e_{\varepsilon}^{+}\right|_{1}^{2}+\left(\mathcal{G}_{u_{\varepsilon}} \frac{\partial e_{\varepsilon}}{\partial t}, e_{\varepsilon}\right)-\frac{1}{2}\left(b^{\prime}\left(u_{\varepsilon}\right) \frac{\partial u_{\varepsilon}}{\partial t} \nabla \mathcal{G}_{u_{\varepsilon}} e_{\varepsilon}, \nabla \mathcal{G}_{u_{\varepsilon}} e_{\varepsilon}\right) \\
&= \gamma\left|e_{\varepsilon}^{+}\right|_{1}^{2}+\left(\mathcal{G}_{u_{\varepsilon}} \frac{\partial e_{\varepsilon}}{\partial t}, e_{\varepsilon}^{+}\right)+\left(\mathcal{G}_{u_{\varepsilon}} \frac{\partial e_{\varepsilon}}{\partial t}, e_{\varepsilon}-e_{\varepsilon}^{+}\right)-\frac{1}{2}\left(b^{\prime}\left(u_{\varepsilon}\right) \frac{\partial u_{\varepsilon}}{\partial t} \nabla \mathcal{G}_{u_{\varepsilon}} e_{\varepsilon}, \nabla \mathcal{G}_{u_{\varepsilon}} e_{\varepsilon}\right) \\
& \leq C_{b}\left[1+\left\|\frac{\partial U_{\varepsilon}}{\partial t}\right\|_{-1}^{2}\right]\left[\left\|e_{\varepsilon}\right\|_{-1}^{2}+\left|e_{\varepsilon}^{A}\right|_{1}^{2}+\varepsilon^{-1}\left|e_{\varepsilon}^{A}\right|_{0}^{2}+\left\|\frac{\partial e_{\varepsilon}}{\partial t}\right\|_{-1}\left|e_{\varepsilon}^{A}\right|_{0}\right. \\
&\left.+\left[(\Delta t)^{2}+h^{4}\right]\left|\frac{\partial U_{\varepsilon}}{\partial t}\right|_{1}^{2}+h^{4}\left\|U_{\varepsilon}^{+}\right\|_{1}^{2}+h^{2}\left\|\pi^{h}\left[\phi_{\varepsilon}\left(U_{\varepsilon}^{+}\right)\right]\right\|_{1}^{2}+(\Delta t)^{2}\left|\frac{\partial W_{\varepsilon}}{\partial t}\right|_{1}^{2}+h^{2}\right] \\
&(3.81) \quad+C \Delta t\left\|\frac{\partial e_{\varepsilon}}{\partial t}\right\|_{-1}\left\|\frac{\partial U_{\varepsilon}}{\partial t}\right\|_{-1}+C_{b}\left|\frac{\partial u_{\varepsilon}}{\partial t}\right|_{0}^{\frac{8}{8-d}}\left\|e_{\varepsilon}\right\|_{-1}^{2} .
\end{aligned}
$$


Integrating (3.81) over $t \in(0, T)$, using a Gronwall inequality and noting (2.13), (2.18), (3.42), (2.46a), (3.6), (3.13), (3.28), (3.5) and (2.46b) yields that

$$
\begin{aligned}
&\left\|e_{\varepsilon}^{+}\right\|_{L^{2}\left(0, T ; H^{1}(\Omega)\right)}^{2}+\left\|e_{\varepsilon}\right\|_{L^{\infty}\left(0, T ;\left(H^{1}(\Omega)\right)^{\prime}\right)}^{2} \\
& \leq C\left(b_{\min }^{-1},\left\|\frac{\partial U_{\varepsilon}}{\partial t}\right\|_{L^{\infty}\left(0, T ;\left(H^{1}(\Omega)\right)^{\prime}\right)},\left\|\frac{\partial u_{\varepsilon}}{\partial t}\right\|_{L^{2}\left(\Omega_{T}\right)}\right)\left[h^{2}+\left\|u_{0}-U_{\varepsilon}^{0}\right\|_{-1}^{2}\right. \\
&+\left\|e_{\varepsilon}^{A}\right\|_{L^{2}\left(0, T ; H^{1}(\Omega)\right)}^{2}+\varepsilon^{-1}\left\|e_{\varepsilon}^{A}\right\|_{L^{2}\left(\Omega_{T}\right)}^{2} \\
&+\left\|\frac{\partial e_{\varepsilon}}{\partial t}\right\|_{L^{2}\left(0, T ;\left(H^{1}(\Omega)\right)^{\prime}\right)}\left[\left|e_{\varepsilon}^{A}\right|_{L^{2}\left(\Omega_{T}\right)}+\Delta t\left\|\frac{\partial U_{\varepsilon}}{\partial t}\right\|_{L^{2}\left(0, T ;\left(H^{1}(\Omega)\right)^{\prime}\right)}\right] \\
&+h^{4}\left\|U_{\varepsilon}^{+}\right\|_{L^{2}\left(0, T ; H^{1}(\Omega)\right)}^{2}+h^{2}\left\|\pi^{h}\left[\phi_{\varepsilon}\left(U_{\varepsilon}^{+}\right)\right]\right\|_{L^{2}\left(0, T ; H^{1}(\Omega)\right)}^{2} \\
&\left.+\left[(\Delta t)^{2}+h^{4}\right]\left\|\frac{\partial U_{\varepsilon}}{\partial t}\right\|_{L^{2}\left(0, T ; H^{1}(\Omega)\right)}^{2}+(\Delta t)^{2}\left\|\frac{\partial W_{\varepsilon}}{\partial t}\right\|_{L^{2}\left(0, T ; H^{1}(\Omega)\right)}^{2}\right] \\
& \leq C_{b}\left[\varepsilon^{-1} h^{2}+\Delta t\right] . \quad \square
\end{aligned}
$$

Theorem 3.2. Let $d \leq 3$ and the assumptions on $u_{0}$ of Theorem 1.1 and the assumptions (A) hold. If b satisfies (1.4a) and $\Delta t \leq 4 \gamma /\left[b_{\max } \theta_{c}^{2}\right]$, then for all $h>0$ such that $\left\|U^{0}\right\|_{0, \infty} \leq 1-\frac{1}{2} \delta$; there exists a unique solution $\left\{U^{n}, W^{n}\right\}_{n=0}^{N}$ to $\left(\mathrm{P}^{h, \Delta t}\right)$ satisfying

$$
\begin{aligned}
& \max _{n=0 \rightarrow N}\left|U^{n}\right|_{1}^{2}+b_{\min }^{2} \sum_{n=1}^{N}\left|U^{n}-U^{n-1}\right|_{1}^{2}+\left|W^{0}\right|_{1}^{2} \\
& \quad+b_{\min } \Delta t \sum_{n=1}^{N}\left[\left|W^{n}\right|_{1}^{2}+\left|\hat{\mathcal{G}}^{h}\left(\frac{U^{n}-U^{n-1}}{\Delta t}\right)\right|_{1}^{2}\right]+b_{\min } \Delta t \sum_{n=1}^{N}\left|\pi^{h}\left[\phi\left(U^{n}\right)\right]\right|_{0}^{2} \leq C
\end{aligned}
$$

and

$$
\|U\|_{L^{\infty}\left(\Omega_{T}\right)}<1 \text {. }
$$

In addition if $d \leq 2, b$ satisfies (1.4b) and $\left|u_{0}-U^{0}\right|_{1}^{2} \leq C \Delta t$, we have that

$$
\begin{aligned}
\Delta t \sum_{n=1}^{N}\left\|\frac{U^{n}-U^{n-1}}{\Delta t}\right\|_{1}^{2}+(\Delta t)^{2} \sum_{n=1}^{N}\left|\frac{W^{n}-W^{n-1}}{\Delta t}\right|_{1}^{2}+\max _{n=0 \rightarrow N}\left|W^{n}\right|_{1}^{2} \\
\quad+\max _{n=1 \rightarrow N}\left|\hat{\mathcal{G}}^{h}\left(\frac{U^{n}-U^{n-1}}{\Delta t}\right)\right|_{1}^{2} \leq C_{b},
\end{aligned}
$$

and for $\varepsilon \leq \varepsilon_{0}$, provided $h^{-\frac{d}{2}}\left[\varepsilon^{-1} h^{2}+\Delta t\right] \leq C$, we have that $(3.85)\left\|U^{+}-U_{\varepsilon}^{+}\right\|_{L^{2}\left(0, T ; H^{1}(\Omega)\right)}^{2}+\left\|U-U_{\varepsilon}\right\|_{L^{\infty}\left(0, T ;\left(H^{1}(\Omega)\right)^{\prime}\right)}^{2} \leq C_{b}\left[\varepsilon+\varepsilon^{-1} h^{2}+\Delta t\right]$.

Here $U$ and $U^{+}$are defined similarly to their regularized counterparts; see Theorem 1.1 .

Proof. The proof is a discrete analogue of Theorem 2.2. Uniqueness of a solution to $\left(\mathrm{P}^{h, \Delta t}\right)$ follows as for $\left(\mathrm{P}_{\varepsilon}^{h, \Delta t}\right)$. Existence of a solution follows by letting $\varepsilon \rightarrow 0$, noting the uniform bounds in (3.28) and applying a discrete analogue of (2.68)(2.69). Hence the bounds (3.82) hold. The bound (3.83) follows immediately from the bound on $\pi^{h}\left[\phi\left(U^{n}\right)\right]$ in (3.82). Similarly the bounds (3.84) hold for $d \leq 2$ on noting the uniform bounds (3.42).

We now prove (3.85). For a.e. $t \in(0, T)$ we set $E_{U}^{( \pm)}:=U^{( \pm)}-U_{\varepsilon}^{( \pm)} \in V^{h}$ and $E_{W}^{( \pm)}:=W^{( \pm)}-W_{\varepsilon}^{( \pm)}$. We note, similarly to (3.66), that

$$
E_{U}^{+}-E_{U}=\left(t_{n}-t\right) \frac{\partial E_{U}}{\partial t} \quad \text { for } \quad t \in\left(t_{n-1}, t_{n}\right]
$$


Subtracting (3.65a) from its non-regularized counterpart, choosing $\chi \equiv E_{U}^{+}$and noting (3.8), (3.60), (3.21), (3.3), (3.10), (3.19) and (2.15) yields for a.e. $t \in(0, T)$ that

$$
\begin{aligned}
\gamma\left|E_{U}^{+}\right|_{1}^{2} & +\left(\phi\left(U^{+}\right)-\phi_{\varepsilon}\left(U_{\varepsilon}^{+}\right), E_{U}^{+}\right)^{h}+\left(\mathcal{G}_{u_{\varepsilon}} \frac{\partial E_{U}}{\partial t}, E_{U}^{+}\right) \\
= & \theta_{c}\left|E_{U}^{+}\right|_{h}^{2}+\left(\frac{\partial E_{U}}{\partial t},\left[\mathcal{G}_{u_{\varepsilon}}-\hat{\mathcal{G}}_{U_{\varepsilon}^{-}}^{h}\right] E_{U}^{+}\right)+\left(\frac{\partial U}{\partial t},\left[\hat{\mathcal{G}}_{U_{\varepsilon}^{-}}^{h}-\hat{\mathcal{G}}_{U^{-}}^{h}\right] E_{U}^{+}\right)^{h} \\
& +\left[\left(\frac{\partial E_{U}}{\partial t}, \hat{\mathcal{G}}_{U_{\varepsilon}^{-}}^{h} E_{U}^{+}\right)-\left(\frac{\partial E_{U}}{\partial t}, \hat{\mathcal{G}}_{U_{\varepsilon}^{-}}^{h} E_{U}^{+}\right)^{h}\right] \\
& +\Delta t\left[\left(\left[b\left(U^{-}\right)-b_{\max }\right] \nabla \frac{\partial W}{\partial t}, \nabla \hat{\mathcal{G}}_{U^{-}}^{h} E_{U}^{+}\right)\right. \\
& \left.-\left(\left[b\left(U_{\varepsilon}^{-}\right)-b_{\max }\right] \nabla \frac{\partial W_{\varepsilon}}{\partial t}, \nabla \hat{\mathcal{G}}_{U_{\varepsilon}^{-}}^{h} E_{U}^{+}\right)\right] \\
\leq & C\left\|E_{U}^{+}\right\|_{-1}^{2}+C\left\|\frac{\partial E_{U}}{\partial t}\right\|_{-1}\left|\left(\mathcal{G}_{u_{\varepsilon}}-\hat{\mathcal{G}}_{U_{\varepsilon}^{-}}^{h}\right) E_{U}^{+}\right|_{1}+C\left\|\frac{\partial U}{\partial t}\right\|_{-1}\left|\left(\hat{\mathcal{G}}_{U_{\varepsilon}^{-}}^{h}-\hat{\mathcal{G}}_{U^{-}}^{h}\right) E_{U}^{+}\right|_{1} \\
& +C_{b}\left\|E_{U}^{+}\right\|_{-1}\left[h^{2}\left|\frac{\partial E_{U}}{\partial t}\right|_{1}+\Delta t\left|\frac{\partial W}{\partial t}\right|_{1}+\Delta t\left|\frac{\partial W_{\varepsilon}}{\partial t}\right|_{1}\right] .
\end{aligned}
$$

Similarly to (3.73) we have that

$$
\left|\left(\mathcal{G}_{u_{\varepsilon}}-\hat{\mathcal{G}}_{u_{\varepsilon}}^{h}\right) E_{U}^{+}\right|_{1} \leq C_{b} h\left|E_{U}^{+}\right|_{0} \leq C_{b}\left[\left\|E_{U}^{+}\right\|_{-1}\left|E_{U}^{+}\right|_{1}+h^{2}\right] .
$$

Similarly to (3.74) and (3.77) we have that

$$
\begin{aligned}
\left|\left(\hat{\mathcal{G}}_{u_{\varepsilon}}^{h}-\hat{\mathcal{G}}_{U_{\varepsilon}^{-}}^{h}\right) E_{U}^{+}\right|_{1} & \leq C_{b}\left|e_{\varepsilon}^{-}\right|_{0,4}\left|\hat{\mathcal{G}}_{u_{\varepsilon}}^{h} E_{U}^{+}\right|_{1,4} \leq C_{b}\left|e_{\varepsilon}^{-}\right|_{0,4}|| E_{U}^{+} \|_{-1}^{1-\frac{d}{8}}\left|E_{U}^{+}\right|_{1}^{\frac{d}{8}} \\
& \leq C_{b}\left[\left\|E_{U}^{+}\right\|_{-1}^{2-\frac{d}{4}}\left|E_{U}^{+}\right|_{1}^{\frac{d}{4}}+\left|e_{\varepsilon}^{+}\right|_{1}^{2}+(\Delta t)^{2}\left|\frac{\partial U_{\varepsilon}}{\partial t}\right|_{1}^{2}\right]
\end{aligned}
$$

where we have noted $(2.10),(2.13)$ and (3.66). Similarly to (3.89) we have that

$$
\begin{aligned}
\mid\left(\hat{\mathcal{G}}_{U_{\varepsilon}^{-}}^{h}\right. & \left.-\hat{\mathcal{G}}_{U^{-}}^{h}\right)\left.E_{U}^{+}\right|_{1} \leq C_{b}\left|E_{U}^{-}\right|_{0,4}\left|\hat{\mathcal{G}}_{U_{\varepsilon}^{-}}^{h} E_{U}^{+}\right|_{1,4} \\
& \leq C_{b}\left|E_{U}^{-}\right|_{0,4}\left[\left|\hat{\mathcal{G}}_{u_{\varepsilon}}^{h} E_{U}^{+}\right|_{1,4}+h^{-\frac{d}{4}}\left|\left(\hat{\mathcal{G}}_{u_{\varepsilon}}^{h}-\hat{\mathcal{G}}_{U_{\varepsilon}^{-}}^{h}\right) E_{U}^{+}\right|_{1}\right] \\
& \leq C_{b}\left[1+h^{-\frac{d}{4}}\left|e_{\varepsilon}^{-}\right|_{0,4}\right]\left|E_{U}^{-}\right|_{0,4}\left|\hat{\mathcal{G}}_{u_{\varepsilon}}^{h} E_{U}^{+}\right|_{1,4} \\
& \leq C_{b}\left[1+h^{-\frac{d}{4}}\left|e_{\varepsilon}^{-}\right|_{0,4}\right]\left\|E_{U}^{+}\right\|_{-1}^{1-\frac{d}{8}}\left|E_{U}^{+}\right|_{1}^{\frac{d}{8}}\left\|E_{U}^{-}\right\|_{-1}^{\frac{4-d}{8}}\left|E_{U}^{-}\right|_{1}^{\frac{4+d}{8}}
\end{aligned}
$$

where we have noted (3.11), (2.10) and (2.15). Similarly to (3.80) we have that

$$
\begin{aligned}
& \left|\left(b^{\prime}\left(u_{\varepsilon}\right) \frac{\partial u_{\varepsilon}}{\partial t} \nabla \mathcal{G}_{u_{\varepsilon}} E_{U}, \nabla \mathcal{G}_{u_{\varepsilon}} E_{U}\right)\right| \\
& \quad \leq \frac{1}{4} \gamma\left|E_{U}^{+}\right|_{1}^{2}+C_{b}\left[\left|\frac{\partial u_{\varepsilon}}{\partial t}\right|_{0}^{\frac{8}{8-d}}\left\|E_{U}\right\|_{-1}^{2}+(\Delta t)^{2}\left|\frac{\partial E_{U}}{\partial t}\right|_{1}^{2}\right] .
\end{aligned}
$$


It follows from (3.87), (2.8), (2.22), (3.86), (3.7), an analogue of (2.72), (2.18), (3.91), (3.88), (3.89), (3.90) and a Young's inequality that for a.e. $t \in(0, T)$

$$
\begin{aligned}
\gamma\left|E_{U}^{+}\right|_{1}^{2} & +\frac{\theta}{2 \varepsilon}\left(E_{U}^{+}, E_{U}^{+}\right)_{\omega_{\varepsilon}^{+}(t) \cup \omega_{\varepsilon}^{-}(t)}^{h}+\frac{1}{2} \frac{\mathrm{d}}{\mathrm{d} t}\left(\mathcal{G}_{u_{\varepsilon}} E_{U}, E_{U}\right) \\
\leq & \gamma\left|E_{U}^{+}\right|_{1}^{2}+\left(\phi_{\varepsilon}\left(U^{+}\right)-\phi_{\varepsilon}\left(U_{\varepsilon}^{+}\right), E_{U}^{+}\right)^{h}+\left(\mathcal{G}_{u_{\varepsilon}} \frac{\partial E_{U}}{\partial t}, E_{U}\right) \\
& -\frac{1}{2}\left(b^{\prime}\left(u_{\varepsilon}\right) \frac{\partial u_{\varepsilon}}{\partial t} \nabla \mathcal{G}_{u_{\varepsilon}} E_{U}, \nabla \mathcal{G}_{u_{\varepsilon}} E_{U}\right) \\
\leq & \left(\phi_{\varepsilon}\left(U^{+}\right)-\phi\left(U^{+}\right), E_{U}^{+}\right)^{h}+\left(\mathcal{G}_{u_{\varepsilon}} \frac{\partial E_{U}}{\partial t}, E_{U}-E_{U}^{+}\right) \\
& -\frac{1}{2}\left(b^{\prime}\left(u_{\varepsilon}\right) \frac{\partial u_{\varepsilon}}{\partial t} \nabla \mathcal{G}_{u_{\varepsilon}} E_{U}, \nabla \mathcal{G}_{u_{\varepsilon}} E_{U}\right)+C\left\|E_{U}^{+}\right\|_{-1}^{2} \\
& +C\left\|\frac{\partial E_{U}}{\partial t}\right\|_{-1}\left|\left(\mathcal{G}_{u_{\varepsilon}}-\hat{\mathcal{G}}_{U_{\varepsilon}^{-}}^{h}\right) E_{U}^{+}\right|_{1}+C\left\|\frac{\partial U}{\partial t}\right\|_{-1}\left|\left(\hat{\mathcal{G}}_{U_{\varepsilon}^{-}}^{h}-\hat{\mathcal{G}}_{U^{-}}^{h}\right) E_{U}^{+}\right|_{1} \\
& +C_{b}\left\|E_{U}^{+}\right\|_{-1}\left[h^{2}\left|\frac{\partial E_{U}}{\partial t}\right|_{1}+\Delta t\left|\frac{\partial W}{\partial t}\right|_{1}+\Delta t\left|\frac{\partial W_{\varepsilon}}{\partial t}\right|_{1}\right] \\
\leq & C \mid\left(\phi\left(U^{+}\right), E_{U}^{+}\right)_{\omega_{\varepsilon}^{+}(t) \cup \omega_{\varepsilon}^{-}(t)}^{h}+C_{b}\left[1+\left|\frac{\partial u_{\varepsilon}}{\partial t}\right|_{0}^{\frac{8}{8-d}}\right]\left\|E_{U}\right\|_{-1}^{2} \\
& +C\left\|\frac{\partial E_{U}}{\partial t}\right\|_{-1}\left|\left(\mathcal{G}_{u_{\varepsilon}}-\hat{\mathcal{G}}_{U_{\varepsilon}^{-}}^{h}\right) E_{U}^{+}\right|_{1}+C\left\|\frac{\partial U}{\partial t}\right\|_{-1}\left|\left(\hat{\mathcal{G}}_{U_{\varepsilon}^{-}}^{h}-\hat{\mathcal{G}}_{U^{-}}^{h}\right) E_{U}^{+}\right|_{1} \\
& +C_{b}\left\{\Delta t\left\|\frac{\partial E_{U}}{\partial t}\right\|_{-1}^{2}+\left[h^{4}+(\Delta t)^{2}\right]\left|\frac{\partial E_{U}}{\partial t}\right|_{1}^{2}+(\Delta t)^{2}\left|\frac{\partial W}{\partial t}\right|_{1}^{2}+(\Delta t)^{2}\left|\frac{\partial W_{\varepsilon}}{\partial t}\right|_{1}^{2}\right\} \\
\leq \quad & C_{b}\left[1+\left\|\frac{\partial E_{U}}{\partial t}\right\|_{-1}^{2}\right]\left[\left\|E_{U}\right\|_{-1}^{2}+h^{2}+\left|e_{\varepsilon}^{+}\right|_{1}^{2}+(\Delta t)^{2}\left\|\frac{\partial E_{U}}{\partial t}\right\|_{-1}^{2}+(\Delta t)^{2}\left|\frac{\partial U_{\varepsilon}}{\partial t}\right|_{1}^{2}\right] \\
& \left.+C_{b}\left\{\left\|\frac{\partial U}{\partial t}\right\|_{-1}\left[1+h^{-\frac{d}{4}}\left|e_{\varepsilon}^{-}\right|_{1}\right]\right\}\right\}^{\frac{8}{6-d}}\left[\left\|E_{U}\right\|_{-1}^{2}+(\Delta t)^{2}\left\|\frac{\partial E_{U}}{\partial t}\right\|_{-1}^{2}\right] \\
& +C \varepsilon\left|\pi^{h}\left[\phi\left(U^{+}\right)\right]\right|_{0}^{2}+C_{b}\left|\frac{\partial u_{\varepsilon}}{\partial t}\right|_{0}^{\frac{8}{8-d}}\left\|E_{U}\right\|_{-1}^{2} \\
& +C_{b}\left\{\Delta t\left\|\frac{\partial E_{U}}{\partial t}\right\|_{-1}^{2}+\left[h^{4}+(\Delta t)^{2}\right]\left|\frac{\partial E_{U}}{\partial t}\right|_{1}^{2}+(\Delta t)^{2}\left|\frac{\partial W}{\partial t}\right|_{1}^{2}+(\Delta t)^{2}\left|\frac{\partial W_{\varepsilon}}{\partial t}\right|_{1}^{2}\right\}
\end{aligned}
$$

where, recalling the notation of (1.6),

$$
\left(\eta_{1}, \eta_{2}\right)_{\omega_{\varepsilon}^{+}(t) \cup \omega_{\varepsilon}^{-}(t)}^{h}:=\sum_{j \in \omega_{\varepsilon}^{+}(t) \cup \omega_{\varepsilon}^{-}(t)} M_{j} \eta_{1}\left(x_{j}\right) \eta_{2}\left(x_{j}\right), \quad \forall \eta_{1}, \eta_{2} \in C(\bar{\Omega}) ;
$$

and $\omega_{\varepsilon}^{+}(t):=\left\{j: 1-\varepsilon \leq U^{+}\left(x_{j}, t\right) \leq U_{\varepsilon}^{+}\left(x_{j}, t\right)\right\}, \omega_{\varepsilon}^{-}(t):=\left\{j: U_{\varepsilon}^{+}\left(x_{j}, t\right) \leq\right.$ $\left.U^{+}\left(x_{j}, t\right) \leq-1+\varepsilon\right\}$. Integrating the above for $t \in(0, T)$, applying a Gronwall inequality and noting (2.13), (2.18), (2.46a), (3.42), (3.64), (3.82) and (3.84) yields that

$$
\begin{aligned}
\left\|E_{U}^{+}\right\|_{L^{2}\left(0, T ; H^{1}(\Omega)\right)}^{2}+\left\|E_{U}\right\|_{L^{\infty}\left(0, T ;\left(H^{1}(\Omega)\right)^{\prime}\right)}^{2} \leq C\left(b_{\min }^{-1},\left\|\frac{\partial U}{\partial t}\right\|_{L^{\infty}\left(0, T ;\left(H^{1}(\Omega)\right)^{\prime}\right)},\right. \\
\left.\quad\left\|\frac{\partial U_{\varepsilon}}{\partial t}\right\|_{L^{\infty}\left(0, T ;\left(H^{1}(\Omega)\right)^{\prime}\right)},\left\|\frac{\partial u_{\varepsilon}}{\partial t}\right\|_{L^{2}\left(\Omega_{T}\right)}, h^{-\frac{d}{4}}\left\|e_{\varepsilon}^{-}\right\|_{L^{2}\left(0, T ; H^{1}(\Omega)\right)}\right) \\
\quad \times\left[h^{2}+\varepsilon\left\|\pi^{h}\left[\phi\left(U^{+}\right)\right]\right\|_{L^{2}\left(\Omega_{T}\right)}^{2}+\Delta t\left\|\frac{\partial E_{U}}{\partial t}\right\|_{L^{2}\left(0, T ;\left(H^{1}(\Omega)\right)^{\prime}\right)}^{2}\right. \\
+\left[(\Delta t)^{2}+h^{4}\right]\left\|\frac{\partial E_{U}}{\partial t}\right\|_{L^{2}\left(0, T ; H^{1}(\Omega)\right)}^{2}+\left\|e_{\varepsilon}^{+}\right\|_{L^{2}\left(0, T ; H^{1}(\Omega)\right)}^{2} \\
\left.\quad+(\Delta t)^{2}\left\{\left\|\frac{\partial U_{\varepsilon}}{\partial t}\right\|_{L^{2}\left(0, T ; H^{1}(\Omega)\right)}^{2}+\left\|\frac{\partial W}{\partial t}\right\|_{L^{2}\left(0, T ; H^{1}(\Omega)\right)}^{2}+\left\|\frac{\partial W_{\varepsilon}}{\partial t}\right\|_{L^{2}\left(0, T ; H^{1}(\Omega)\right)}^{2}\right\}\right] \\
\leq C_{b}\left[\varepsilon+\varepsilon^{-1} h^{2}+\Delta t\right],
\end{aligned}
$$

since using (3.66)

$$
\begin{aligned}
& h^{-\frac{d}{4}}\left\|e_{\varepsilon}^{-}\right\|_{L^{2}\left(0, T ; H^{1}(\Omega)\right)} \leq C h^{-\frac{d}{4}}\left[\left\|e_{\varepsilon}^{+}\right\|_{L^{2}\left(0, T ; H^{1}(\Omega)\right)}+\Delta t\left\|\frac{\partial U_{\varepsilon}}{\partial t}\right\|_{L^{2}\left(0, T ; H^{1}(\Omega)\right)}\right] \\
& \quad \leq C_{b} h^{-\frac{d}{4}}\left[\varepsilon^{-1} h^{2}+\Delta t\right]^{1 / 2} \leq C_{b} .
\end{aligned}
$$

Hence the desired result (3.85) follows. 
Therefore combining (2.67), (3.64) and (3.85) yields that

$$
\begin{aligned}
& \left\|u-U^{+}\right\|_{L^{2}\left(0, T ; H^{1}(\Omega)\right)}^{2}+\|u-U\|_{L^{\infty}\left(0, T ;\left(H^{1}(\Omega)\right)^{\prime}\right)}^{2} \\
& \quad \leq C_{b}\left[\varepsilon+\varepsilon^{-1} h^{2}+\Delta t\right] \leq C_{b}\left[\varepsilon+\varepsilon^{-1} h^{2}+h\right],
\end{aligned}
$$

provided $\Delta t \equiv C_{1} h \leq 4 \gamma /\left[b_{\max } \theta_{c}^{2}\right], h^{-\frac{d}{2}}\left[\varepsilon^{-1} h^{2}+\Delta t\right] \leq C_{b}$ and $U_{\varepsilon}^{0} \equiv U^{0}$ satisfies (1.9). Hence choosing $\varepsilon \equiv C_{2} h \leq \varepsilon_{0}$, for some constant $C_{2}$ proves Theorem 1.1. Finally we note that on choosing $U^{0} \equiv Q^{h} u_{0}$, the second bound in (1.9) is satisfied for all $h>0$ and a sufficient condition for the first to hold is that $h$ is sufficiently small; see (3.6) and (3.12). On choosing $U^{0} \equiv \hat{Q}^{h} u_{0}$ the first bound in (1.9) is satisfied for all $h>0$ and the second bound holds with further restrictions on $\mathcal{T}^{h}$ and $u_{0}$; e.g., on a uniform translation invariant mesh if $u_{0} \in W^{2, \infty}(\Omega)$; see (3.14), (3.15), the first bound in (3.21) and (3.5).

\section{NumericAl EXPERIMENTS}

4.1. One space dimension. As no exact solution to $(\mathrm{P})$ is known, a comparison between the solutions of $\left(\mathrm{P}^{h, \Delta t}\right)$ on a coarse mesh, $U$, with that on a fine mesh, $u$, was made. The data used in each experiment on the coarse meshes were $\Omega=(0,1)$, $\gamma=1.5 \times 10^{-3}, \theta=0.3, \theta_{c}=1.0, T=0.4, \Delta t=0.32 h, h=1 /(\mathcal{J}-1)$, where $\mathcal{J}=$ $2^{k}+1(k=6,7,8,9), b_{\max }=1$, tol $=1 \times 10^{-7}$ and $\mu=0.1$. The last two quantities were parameters used to vary the degree and speed of convergence in the iterative method (method II of [10]) to solve for $U^{n}$ at each time level in $\left(\mathrm{P}^{h, \Delta t}\right)$. The data were the same for the fine mesh except $\mathcal{J}=2^{12}+1$. We note that the restriction on $\Delta t$ in Theorem 1.1 holds for this data. The initial data $u_{0}$ was taken to be the clamped (complete) cubic spline interpolating $\frac{1}{5}(\cos (\pi x)-\cos (3 \pi x)+1.3 \cos (5 \pi x))$ at the points $i / 8(i=0 \rightarrow 8)$. Hence we have that

$$
u_{0} \in H^{3}(\Omega) \backslash H^{4}(\Omega) \text { and } u_{0}^{\prime}(0)=u_{0}^{\prime}(1)=0 .
$$

On setting $U^{0} \equiv \hat{Q}^{h} u_{0}$, it follows that the assumptions on $u_{0}$ and $U^{0}$ of Theorem 1.1 hold. In addition this choice of initial data $u_{0}$ ensured that the singularities in $\phi$ played a role. We performed three experiments with $b$ given by (1.3) with $\sigma=0.5,0.8$ and 0.9. For this choice of $b$, the integral on the right hand side of (1.8a) can be evaluated exactly using Simpson's rule on each element. In Figure 1, we plot $u_{\sigma}(\cdot, 0)$ and $u_{\sigma}(\cdot, 0.4)$, where $u_{\sigma}$ denotes the "true solution" of $(\mathrm{P})$ dependent on $\sigma$.

\begin{tabular}{|c|c|c|c|c|}
\hline $\begin{array}{ll}\sigma & \mathcal{J}\end{array}$ & 65 & 129 & 257 & 513 \\
\hline$\overline{0.5}$ & 8.88 & 2.12 & $\overline{0.470}$ & $9.63 \times 10^{-2}$ \\
\hline 0.8 & 0.130 & $3.05 \times 10^{-2}$ & $7.21 \times 10^{-3}$ & $1.59 \times 10^{-3}$ \\
\hline 0.9 & $8.35 \times 10^{-2}$ & $1.88 \times 10^{-2}$ & $4.41 \times 10^{-3}$ & $9.72 \times 10^{-4}$ \\
\hline
\end{tabular}

The quantity $E^{2}:=\Delta t \sum_{n=1}^{N}\left|\pi^{h} u^{n}-U^{n}\right|_{1}^{2}$ was computed and is given in the table below.

We see that the ratio of consecutive $E^{2}$ are between 4.2 and 4.9 which is better than 2 , the rate of convergence proved in Theorem 1.1.

It is interesting to consider the effect of varying $b_{\max }$ on the numerical solution. We performed several numerical experiments for $b(s) \equiv 1$ with precisely the same parameters and data as mentioned above except $h=10^{-2}, U^{0}=-0.6 \pm \delta^{h}$ where $\delta^{h} \in S^{h}$ with $\left\|\delta^{h}\right\|_{0, \infty} \leq 0.05, W^{0}$ satisfying (1.8b) when $n=0$ and $\Delta t=2^{i} \times 10^{-4}$ 


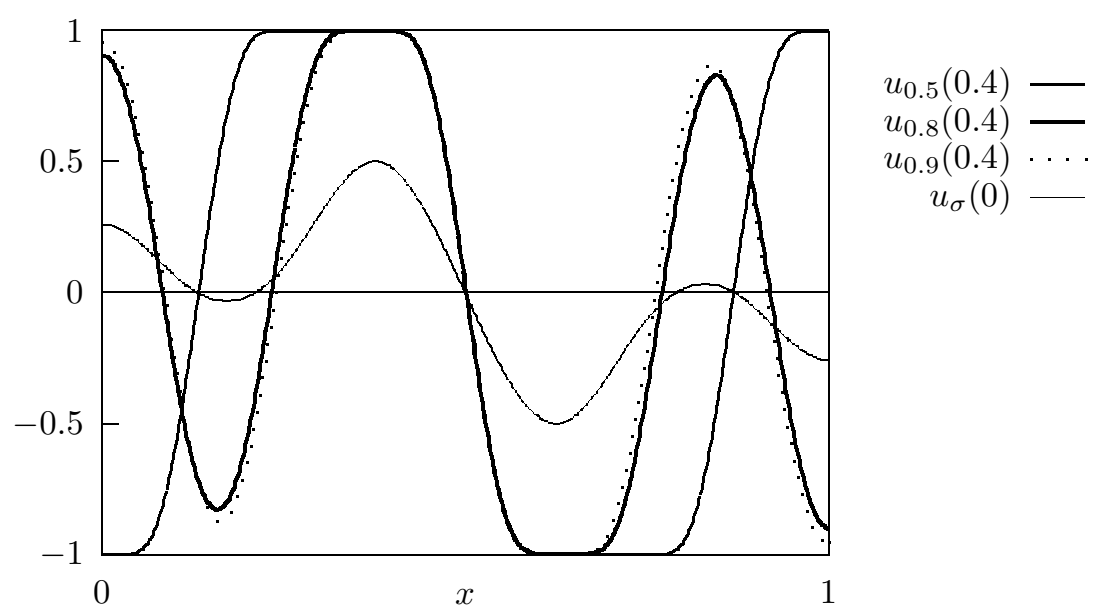

FiguRE 1. $u_{\sigma}$ plotted for $\sigma=0.5,0.8,0.9$

with $i=-1,0,1$ and 2 . We took $b_{\max }=1,2$ and 4 . Once again the restriction on $\Delta t$ in Theorem 1.1 holds for this data. For a fixed $b_{\max }$, the solutions for the different choices of $\Delta t$ were graphically indistinguishable. However for a fixed $\Delta t$, the solutions for the different choices of $b_{\max }$ were distinguishable in that they had similar dynamics with a time delay. As $\Delta t$ decreased this delay decreased as one would expect. Repeating the experiment above for fixed $b_{\max }$ with the alternative time stepping scheme, where $\theta_{c} U^{n}$ in $(1.8 \mathrm{~b})$ is replaced by $\theta_{c} U^{n-1}$ as mentioned in the introduction, one obtains distinguishable solutions as $\Delta t$ is varied. Thus we prefer the discretization $\left(\mathrm{P}^{h, \Delta t}\right)$.

4.2. Two space dimensions. We performed several numerical experiments in two spatial dimensions with $\Omega=(0,1) \times(0,1)$. We took a uniform mesh consisting of squares $\kappa$ of length $h=1 / 64$, each of which was divided into two triangles by its north-east diagonal. Instead of (1.6), we used the discrete inner product on $C(\bar{\Omega})$ given by

$$
\left(\eta_{1}, \eta_{2}\right)^{h}=\int_{\Omega} \Pi^{h}\left(\eta_{1}(x) \eta_{2}(x)\right) \mathrm{d} x \quad \forall \eta_{1}, \eta_{2} \in C(\bar{\Omega}),
$$

where $\Pi^{h}$ is the piecewise continuous bilinear interpolant at the vertices on each square $\kappa$. With this choice of discrete inner product all of the results proved in Section 3 still hold and in addition one can exploit "the discrete cosine transform" in solving the nonlinear algebraic system arising at each time level in $\left(\mathrm{P}^{h, \Delta t}\right)$; see [4] for a fuller discussion. The data was taken to be the same as for the first experiment except $\gamma=3.2 \times 10^{-3}=\Delta t$ and $b$ as in (1.3) but with different values for $\sigma$. With $b_{\max }=1$, the restriction on $\Delta t$ in Theorem 1.1 holds. For the above choice of $b$, the integral on the right hand side of (1.8a) can be evaluated exactly by sampling at the midpoints of the sides over each element. The initial data was taken to be $U_{0}= \pm \delta^{h}$, where $\delta^{h} \in S^{h}$ with $\left\|\delta^{h}\right\|_{0, \infty} \leq 0.05$ and $W^{0}$ satisfying (1.8b) when $n=0$. In Figures 2 and 3 we plot a grey scale grid plot of $U$ at several times where the final numerical solution plotted is stationary, that is $U^{n}$ does not change from one time level to the next. The pictures are arranged in a matrix format with time increasing to the right in rows then down columns. The grey scale ranges from 

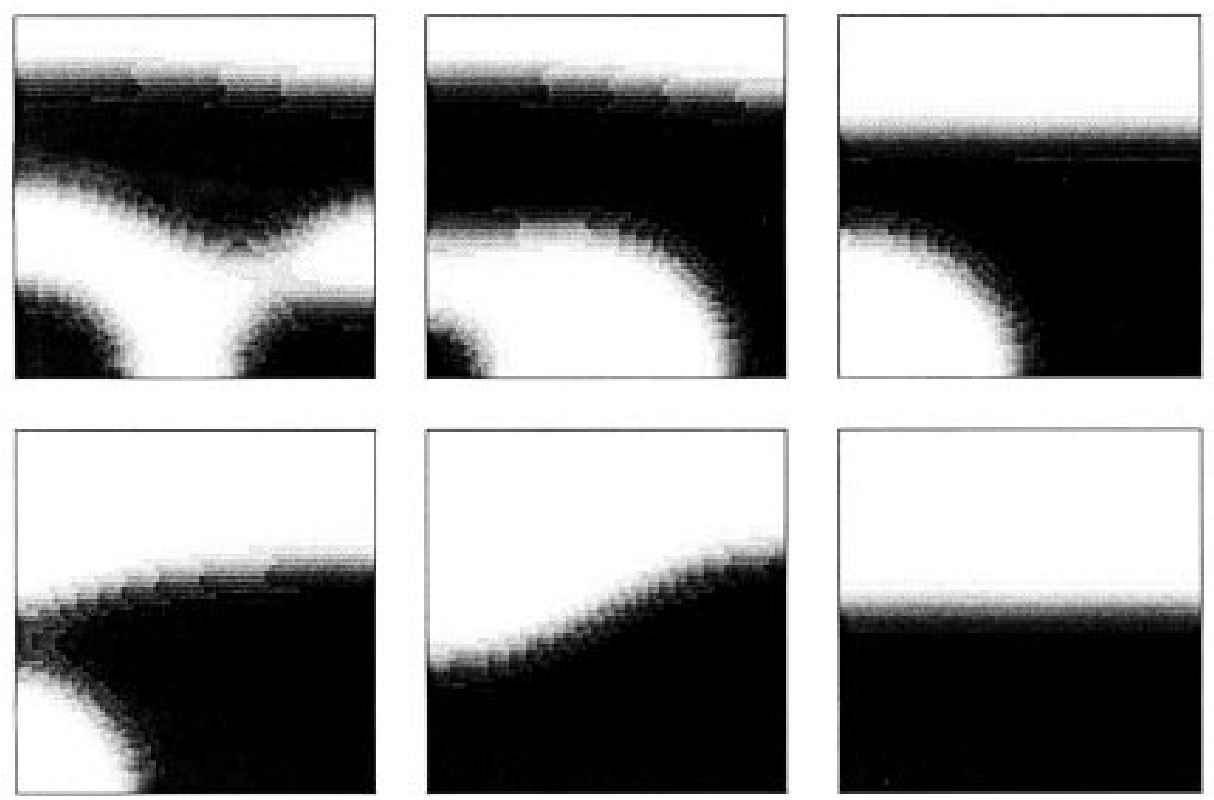

Figure 2. $U_{0.5}(\cdot, t)$ plotted when $t=0.4,0.8,4.0,5.2,7.2$ and 22.4
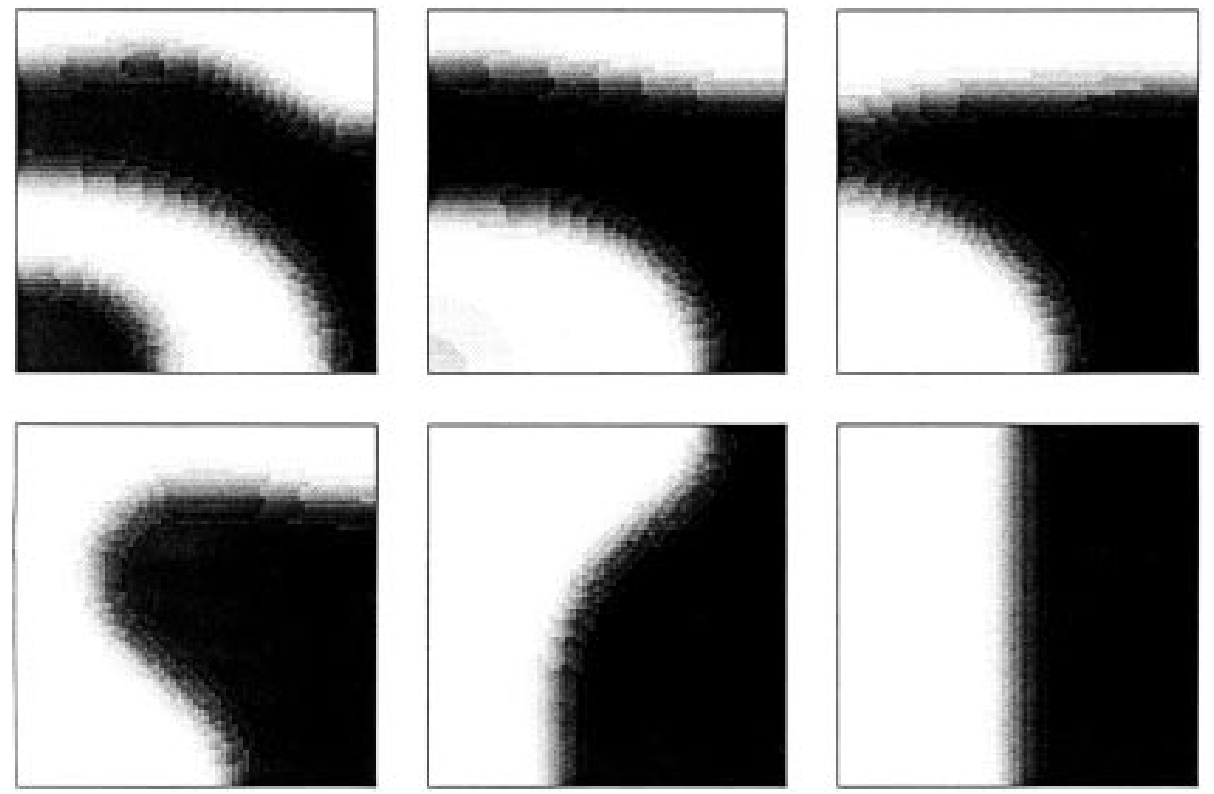

Figure 3. $U_{0.99}(\cdot, t)$ plotted when $t=0.8,4.8,13.6,14.4,16.8$ and 45.6

-0.9 to 0.9 in steps of 0.2 with pure black/white representing values larger/smaller than $0.9 /-0.9$. The largest value of $U_{0.5}(\cdot, 22.4)$ and $U_{0.99}(\cdot, 45.6)$ in magnitude are 0.99741 and 0.99742 correct to 5 d.p. One does not expect these values to depend on $\sigma$. 
From the experiments displayed in Figures 2 and 3, the degeneracy of the mobility $b$ appears to play a crucial role in the behaviour of the numerical solution. Further, as we increase the value of $\sigma$ to 0.999 and then 1.0, making the mobility fully degenerate, we found that the numerical solutions were extremely similar to that obtained with $\sigma=0.9$ with the exception that the pictures appeared to be slightly delayed in time. For $b$ constant, it is clearly seen that decreasing $b$ simply increases the time scale of $(\mathrm{P})$ proportionately. Similarly, comparing the graphs from Figure 1 for $\sigma=0.5$ and 0.9 one infers that for values of $\sigma$ close to 1 , when $b$ is more degenerate, there is a slower time scale.

For a parameter $\epsilon>0$, taking the scalings $\gamma=\epsilon^{2}, \tau=\epsilon^{2} t, b(s)=\left[1-s^{2}\right]_{+}$and letting $\theta=\theta(\epsilon) \searrow 0,[7]$ have shown using a formal asymptotic analysis that the level set

$$
\Gamma(t)=\{x \in \Omega: u(x, t ; \epsilon)=0\}
$$

where $u(x, t ; \epsilon)$ is the solution to $(\mathrm{P})$ dependent on the parameter $\epsilon$, approximately moves in its normal direction with velocity proportional to the surface diffusion. This contrasts with the case where $b(s) \equiv 1$ in which case the level set approximates a Mullins-Sekerka flow. In the experiments shown in Figures 2 and 3 above the values of $\theta$ and $\epsilon$ are not particularly small. However, we wish to exploit this link with surface diffusion in future work.

\section{REFERENCES}

1. R.A. Adams and J. Fournier, Cone conditions and properties of Sobolev spaces, J. Math. Anal. App. 61 (1977), 713-734. MR 57:3840

2. J.W. Barrett and J.F. Blowey, An error bound for the finite element approximation of the Cahn-Hilliard with logarithmic free energy, Numer. Math. 72 (1995), 1-20. MR 96h:65118

3. J.W. Barrett and J.F. Blowey, An error bound for the finite element approximation of a model for phase separation of a multi-component alloy, IMA J. Numer. Anal. 16 (1996), 257-287. MR 97b:73011

4. J.W. Barrett and J.F. Blowey, Finite element approximation of a model for phase separation of a multi-component alloy with non-smooth free energy, Numer. Math. 77 (1997), 1-34. CMP 97:16

5. J.F. Blowey and C.M. Elliott, The Cahn-Hilliard gradient theory for phase separation with non-smooth free energy. I. Mathematical analysis, Eur. Jnl. of Applied Mathematics 2 (1991), 233-279. MR 93a:35025

6. J.F. Blowey and C.M. Elliott, The Cahn-Hilliard gradient theory for phase separation with non-smooth free energy. II. Numerical analysis, Eur. Jnl. of Applied Mathematics 3 (1992), 147-179. MR 93g:80007

7. J.W. Cahn, C.M. Elliott and A. Novick-Cohen, The Cahn-Hilliard equation with a concentration dependent mobility: motion by minus the Laplacian of the mean curvature, Eur. Jnl. of Applied Mathematics 7 (1996), 287-301. MR 97g:80010

8. J.W. Cahn and J.E. Hilliard, Spinodal decomposition: A reprise, Acta Metall. 19 (1971), 151-161.

9. J.F. Cialvaldini, Analyse numérique d'un problème de Stefan à deux phases par une méthode d'elements finis, SIAM J. Numer. Anal. 12 (1975), 464-487.

10. M.I.M. Copetti and C.M. Elliott, Numerical analysis of the Cahn-Hilliard equation with logarithmic free energy, Numer. Math. 63 (1992), 39-65. MR 94b:65130

11. Ha Dang, Stability and boundary layer properties of Cahn-Hilliard equations, Ph.D. Thesis, University of Utah (1995).

12. C.M. Elliott, Error analysis of the enthalpy method, IMA J. Numer. Anal. 7 (1987), 61-71. MR 90a:65222

13. C.M. Elliott and H. Garcke, On the Cahn-Hilliard equation with degenerate mobility, SIAM J. Math. Anal. 27 (1996), 404-423. MR 97c:35081 
14. C.M. Elliott and H. Garcke, Diffusional phase transitions in multicomponent systems with a concentration dependent mobility matrix, Physica D 109 (1997) pp. 242-256. CMP 98:06

15. C.M. Elliott and S. Larsson, A finite element model for the time-dependent joule heating problem, Math. Comp. 64 (1995), 1433-1453. MR 95m:65164

16. C.M. Elliott and S. Luckhaus, A generalised diffusion equation for phase separation of a multicomponent mixture with interfacial free energy. IMA, University of Minnesota, Preprint 887 (1991).

17. Yin Jingxue, On the existence of nonnegative continuous solutions of the Cahn-Hilliard equation. J. Diff. Eqns. 97 (1992), 310-327. MR 93f:35193

18. J.L. Lions, Quelques méthodes de résolution des problèmes aux limites nonlinéaires. Dunod, Paris (1969). MR 41:4326

19. R.H. Nochetto, Finite element methods for parabolic free boundary problems, in Advances in numerical analysis vol 1, (W. Light ed.), O.U.P. (1991), pp. 34-95. CMP 92:04

Department of Mathematics, Imperial College, London SW7 2BZ, U.K.

E-mail address: j.barrett@ic.ac.uk

Department of Mathematical Sciences, University of Durham, Durham DH1 3LE, U.K.

E-mail address: j.f.blowey@durham.ac.uk 